F. Bethuel $\cdot$ G. Orlandi $\cdot$ D. Smets

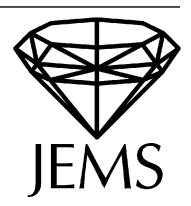

\title{
Vortex rings for the Gross-Pitaevskii equation
}

Received November 4, 2002

\begin{abstract}
We provide a mathematical proof of the existence of traveling vortex rings solutions to the Gross-Pitaevskii (GP) equation in dimension $N \geq 3$. We also extend the asymptotic analysis of the free field Ginzburg-Landau equation to a larger class of equations, including the GinzburgLandau equation for superconductivity as well as the traveling wave equation for GP. In particular we rigorously derive a curvature equation for the concentration set (i.e. line vortices if $N=3$ ).
\end{abstract}

Keywords. NLS, Gross-Pitaevskii, Ginzburg-Landau, vortex rings

\section{Introduction}

In this paper, we consider the Gross-Pitaevskii equation

$$
i \frac{\partial \psi}{\partial t}+\Delta \psi+\left(1-|\psi|^{2}\right) \psi=0
$$

where $\psi: \mathbb{R}^{N} \times \mathbb{R} \rightarrow \mathbb{C}$ and $N \geq 3$. In dimension 3, this equation, or its close variants, are often used as models in various areas of physics: nonlinear optics, superfluidity, BoseEinstein condensation (see e.g. [21, 35, 38] for surveys). At least formally, it possesses a Hamiltonian structure, whose energy is given by

$$
E(\psi):=\frac{1}{2} \int_{\mathbb{R}^{N}}|\nabla \psi(\cdot, t)|^{2}+\frac{1}{4} \int_{\mathbb{R}^{N}}\left(1-|\psi(\cdot, t)|^{2}\right)^{2} .
$$

Another important quantity conserved by the flow 1 is the momentum $\mathbf{P} \in \mathbb{R}^{N}$, given, again formally, by

$$
\mathbf{P}(\psi):=\operatorname{Im} \int_{\mathbb{R}^{N}} \psi \cdot \overline{\nabla \psi}=\int_{\mathbb{R}^{N}}(i \psi, \nabla \psi),
$$

F. Bethuel: Laboratoire Jacques-Louis Lions, Université de Paris 6, 4 place Jussieu, BC 187, 75252 Paris, France; e-mail: bethuel@ann.jussieu.fr

G. Orlandi: Dipartimento di Informatica, Università di Verona, Strada le Grazie, 37134 Verona, Italy; e-mail: orlandi@ @ sci.univr.it

D. Smets: Laboratoire Jacques-Louis Lions, Université de Paris 6, 4 place Jussieu BC 187, 75252 Paris, France; e-mail: smets@ann.jussieu.fr

Mathematics Subject Classification (2000): 35J20, 35J25, 35J60, 35J50, 35Qxx, 49Q05, 49Q15, 49Q20, 53Z05, 58E15, 58E20, 81T13 
where $(\cdot, \cdot)$ stands for the scalar product in $\mathbb{R}^{2}$. The first component of the vector $\mathbf{P}$ will be denoted by $P$, i.e. $P=\mathbf{P} \cdot \mathbf{e}_{1}$.

Traveling wave solutions to (1) are known to play an important role in the full dynamics of (1). More precisely, these are solutions of (1) of the form (up to rotation)

$$
\psi(x, t)=U\left(x_{1}-C t, x_{2}, \ldots, x_{N}\right),
$$

where $C>0$ is the wave's speed and $U: \mathbb{R}^{N} \rightarrow \mathbb{C}$. One easily verifies that $\psi$ is a solution of (1) iff the "profile" $U$ is a solution to the equation

$$
i C \frac{\partial U}{\partial x_{1}}=\Delta U+U-|U|^{2} U
$$

The focus of this paper is on finite energy solutions to (5). Our purpose is twofold. First, we embed equation (5) in a larger class of equations (which contain in particular the equations of superconductivity) and study qualitative properties of solutions in an asymptotic regime which is described below. Since these results are of independent interest (and will be used in forthcoming works), we devote a large appendix to this analysis. It will then enter in a crucial way in our second scope, namely the existence problem for (5). The existence of solutions in the case $N=2$ was considered in [14]; our main existence result here concerns its extension to higher dimensions. For that purpose, consider in cylindrical coordinates $\left(x_{1}, r, \theta\right)$, where $r:=\left(x_{2}^{2}+\cdots+x_{N}^{2}\right)^{1 / 2}$, the sphere $S:=\{(0,1, \theta)\}$, and on the upper half-plane $H_{+}:=\left\{\left(x_{1}, r\right): r>0\right\}$, the operator

$$
L \Psi=r^{N-2} \partial_{r}\left(r^{2-N} \partial_{r} \Psi\right)+\partial_{x_{1}}^{2} \Psi
$$

The linear problem

$$
\left\{\begin{array}{l}
-L \Psi=2 \pi \delta_{q}, \quad q=(0,1), \\
\Psi\left(x_{1}, 0\right)=0,
\end{array}\right.
$$

has a unique solution $\Psi_{*}$ bounded at infinity. Up to a phase change, there also exists (see e.g. [9]) a unique function $\omega_{*} \in \mathcal{C}^{\infty}\left(H_{+} \backslash\{q\}\right)$ such that $\left|\omega_{*}\right|=1$ and

$$
\left(\omega_{*} \times \frac{\partial \omega_{*}}{\partial x_{1}}, \omega_{*} \times \frac{\partial \omega_{*}}{\partial r}\right)=\left(-\frac{\partial \Psi_{*}}{\partial r}, \frac{\partial \Psi_{*}}{\partial x_{1}}\right)
$$

(here $a \times b:=a_{1} b_{2}-a_{2} b_{1}$ is the exterior product of two vectors $a, b \in \mathbb{R}^{2} \simeq \mathbb{C}$ ). Finally, we consider the function $U_{*}$ defined by

$$
U_{*}\left(x_{1}, r, \theta\right):=\omega_{*}\left(x_{1}, r\right) .
$$

The function $U_{*}$ is cylindrically symmetric, smooth on $\mathbb{R}^{N} \backslash S$, with values in the circle $S^{1}$. In particular, in dimension 3, $U_{*}$ is singular on a circle (often referred to as a "concentrated vortex ring"). Our main result states that, after scalings, there are solutions of (5) close to $U_{*}$.

Theorem 1. There exists $\varepsilon_{0}>0$ such that for every $0<\varepsilon<\varepsilon_{0}$ there exists a solution $U_{\varepsilon}$ to $[5]$ with $C=C(\varepsilon)$ satisfying

$$
\frac{C(\varepsilon)}{\varepsilon|\log \varepsilon|} \rightarrow N-2 \quad \text { as } \varepsilon \rightarrow 0
$$


and for $E(\varepsilon):=E\left(U_{\varepsilon}\right)$ and $P(\varepsilon):=P\left(U_{\varepsilon}\right)$ we have

$$
\frac{P(\varepsilon)}{2 \pi \varepsilon^{1-N}}=\left|B^{N-1}\right|, \quad \frac{E(\varepsilon)}{\pi \varepsilon^{2-N}|\log \varepsilon|} \rightarrow\left|S^{N-2}\right|,
$$

and

$$
\left|U_{\varepsilon}(x)\right| \rightarrow 1 \quad \text { as }|x| \rightarrow \infty .
$$

Moreover, for every $k \in \mathbb{N}$,

$$
U_{\varepsilon}(x / \varepsilon) \rightarrow U_{*} \quad \text { in } \mathcal{C}_{\text {loc }}^{k}\left(\mathbb{R}^{N} \backslash S\right) .
$$

Remark 1. Notice that both the energy $E(\varepsilon)$ and the momentum $P(\varepsilon)$ diverge as $\varepsilon \rightarrow 0$, and instead that $C(\varepsilon) \rightarrow 0$ as $\varepsilon \rightarrow 0$.

A few comments are in order. First, observe that (1) corresponds to a defocusing nonlinear Schrödinger equation (NLS); it has been widely studied with respect to the Cauchy problem in case the initial data are in $L^{2}\left(\mathbb{R}^{N}\right)$ (see e.g. [41]). In this (different) situation, due to dispersion, any solution vanishes as time tends to infinity. This phenomenon of course excludes traveling wave solutions except for the trivial one. Instead, in our situation, the $L^{2}$-norm is not bounded (this is incompatible with the fact that $E$ is bounded) for the solution $U_{\varepsilon}$; we have seen that $\left|U_{\varepsilon}(x)\right| \rightarrow 1$ as $|x| \rightarrow \infty$, and dispersion effects are balanced by the nonlinearity. Our results provide some rigorous mathematical proofs to the study in [30].

Second, the Cauchy problem for 11 with an initial data in $H^{1}\left(\mathbb{R}^{N}\right)+\{1\}$ having its vorticity concentrating on round spheres has been considered by Jerrard [28]. Although our results are of a different nature, some of the arguments there are closely related to ours.

Third, some properties of (11) can be usefully analyzed through the Madelung transform

$$
\psi(x, t)=\sqrt{\rho} \exp (i \varphi),
$$

which is meaningful if $|\psi|$ is not zero. In the $\rho$ and $v:=\nabla \varphi$ variables, equation (1) can be written as

$$
\left\{\begin{array}{l}
\frac{\partial \rho}{\partial t}+\operatorname{div}(\rho v)=0, \\
\rho\left(\frac{\partial v}{\partial t}+v \cdot \nabla v\right)+\nabla \rho^{2}=-\rho \nabla\left(\frac{|\nabla \rho|^{2}}{8 \rho^{2}}-\frac{\Delta \rho}{4 \rho}\right) .
\end{array}\right.
$$

If we neglect the term on the right-hand side of 10 ) (which is often termed the "quantum pressure" in the physics literature), this system reduces to the Euler equations for compressible ideal fluids with pressure given by $\rho^{2}$. The full system 10 enters the larger class of quantum fluid equations (see e.g. [36]).

The existence of traveling wave solutions for the incompressible Euler equations was already considered by Helmholtz in his celebrated paper of 1858 [27]; more precisely, the solutions he proposed have vorticity concentrated on a ring of small cross-section (like "smoke rings"). Later, Lord Kelvin computed the relations between the cross-section, the radius of the ring, and its propagation speed. The first rigorous proofs of existence of such 
steady vortex rings (steady in a traveling frame) were given by Fraenkel and Berger [22] in the seventies, and later by Ambrosetti and Struwe [4]. Concerning the compressible Euler equation, we are only aware of numerical results in this direction [33].

We will turn later to the properties of the solutions in Theorem 1 In view of the last statement of the theorem, it is clear that they behave like vortex rings. The remainder of this introduction is a detailed description of the strategy of the analysis.

\subsection{The variational approach}

Since, as already mentioned, (1) is Hamiltonian, it follows that (5) is variational. At least two different variational approaches are available. First, as considered in [14], one could introduce the Lagrangian

$$
F_{C}(U):=E(U)-C P(U),
$$

whose critical points are solutions to (5). This approach has the advantage that the wave speed $C$ is prescribed a priori. It was shown in [14], for $N=2$, that for some $C_{0}>0, F_{C}$ has the mountain-pass geometry for $C<C_{0}$, providing existence in a full interval of speed $] 0, C_{0}$ [. In this approach, however, the question of stability seems more difficult to address.

The second possible approach, the one we will use here, is by minimizing the energy $E$ keeping the momentum $P$ fixed. It is convenient to perform the following rescaling for $0<\varepsilon<1 / 2$ :

$$
u_{\varepsilon}(x):=U_{\varepsilon}\left(\frac{x}{\varepsilon}\right), \quad c(\varepsilon):=\frac{C(\varepsilon)}{\varepsilon|\log \varepsilon|},
$$

so that if $U_{\varepsilon}$ is a solution of (5), then $u_{\varepsilon}$ solves the equation

$$
i c(\varepsilon)|\log \varepsilon| \frac{\partial u_{\varepsilon}}{\partial x_{1}}=\Delta u_{\varepsilon}+\frac{1}{\varepsilon^{2}} u_{\varepsilon}\left(1-\left|u_{\varepsilon}\right|^{2}\right),
$$

and

$$
E_{\varepsilon}\left(u_{\varepsilon}\right):=\varepsilon^{2-N} E\left(U_{\varepsilon}\right)=\int_{\mathbb{R}^{N}}\left(\frac{1}{2}\left|\nabla u_{\varepsilon}\right|^{2}+\frac{1}{4 \varepsilon^{2}}\left(1-\left|u_{\varepsilon}\right|^{2}\right)^{2}\right) \equiv \int_{\mathbb{R}^{N}} e_{\varepsilon}\left(u_{\varepsilon}\right) .
$$

The energy $E_{\varepsilon}$ is often called the Ginzburg-Landau energy, and has been extensively studied, in particular in the asymptotic limit $\varepsilon \rightarrow 0$ (see e.g. [9]). Likewise, the momentum rescales as

$$
\mathbf{p}\left(u_{\varepsilon}\right):=\varepsilon^{1-N} P\left(U_{\varepsilon}\right)=\int_{\mathbb{R}^{N}}\left(i u_{\varepsilon}, \nabla u_{\varepsilon}\right) .
$$

One major difficulty comes from the fact that in the natural energy space

$$
X:=\left\{u \in H_{\mathrm{loc}}^{1}\left(\mathbb{R}^{N}\right): E_{\varepsilon}(u)<\infty\right\},
$$

the momentum $\mathbf{p}$ is not well defined. Indeed, consider for example the function $w:=$ $\exp (i \varphi)$, where $\varphi$ is smooth and $\varphi(x)=|x|^{\alpha}$ for some $(1-N) / 2<\alpha<(2-N) / 2$ and $|x|>1$. Notice that $|w|=1$ and $|\nabla w|=|\nabla \varphi| \in L^{2}\left(\mathbb{R}^{N}\right)$ so that $w \in X$. On the other hand, $(i w, \nabla w)=\nabla \varphi \notin L^{1}\left(\mathbb{R}^{N}\right)$ and similarly $(i(w-1), \nabla w) \notin L^{1}\left(\mathbb{R}^{N}\right)$; hence $\mathbf{p}(w)$ is not well defined in the Lebesgue sense. To overcome this difficulty, we will introduce a series of approximate problems $\left(\mathcal{P}_{n}^{\varepsilon}\right)$ on expanding tori. A price has to be paid, however: 
- one has to find uniform bounds for both the Lagrange multipliers and the solutions associated to $\left(\mathcal{P}_{n}^{\varepsilon}\right)$,

- some information (energy, momentum, ...) could be lost in the limit (see the discussion on stability later).

\subsection{The approximating problems}

Setting. For $n \in \mathbb{N}^{*}$, consider the flat torus

$$
\Pi_{n} \simeq \Omega_{n} \equiv[-n, n]^{N},
$$

with opposite faces identified, and the space

$$
X_{n}:=H^{1}\left(\Pi_{n}, \mathbb{C}\right) \simeq H_{\mathrm{per}}^{1}\left(\Omega_{n}, \mathbb{C}\right)
$$

of $2 n$-periodic $H^{1}$ functions. Since $\Pi_{n}$ is compact, we can define the (first component of the) momentum as

$$
p(u):=\int_{\Pi_{n}}\left(i u, \partial_{1} u\right),
$$

and this clearly defines a quadratic functional on $X_{n}$. Let

$$
\Gamma_{n}:=\left\{u \in X_{n}: p(u)=2 \pi\left|B^{N-1}\right|\right\},
$$

and consider the minimization problem:

$$
\left(\mathcal{P}_{n}^{\varepsilon}\right) \quad I_{n, \varepsilon}:=\inf _{u \in \Gamma_{n}} E_{\varepsilon}(u) .
$$

The constraint is easily seen to be nonvoid. It is also straightforward to prove existence of a minimizer for $\left(\mathcal{P}_{n}^{\varepsilon}\right)$.

Proposition 1. There exists a minimizer $u_{n, \varepsilon} \in X_{n}$ for $\left(\mathcal{P}_{n}^{\varepsilon}\right)$ and some constant $c_{n, \varepsilon} \in \mathbb{R}$ such that $u_{n, \varepsilon}$ satisfies $[11$, i.e.

$$
i c_{n, \varepsilon}|\log \varepsilon| \frac{\partial u_{n, \varepsilon}}{\partial x_{1}}=\Delta u_{n, \varepsilon}+\frac{1}{\varepsilon^{2}} u_{n, \varepsilon}\left(1-\left|u_{n, \varepsilon}\right|^{2}\right) \quad \text { on } \Pi_{n} .
$$

In what follows, for simplicity, we will skip the subscripts $n$ or $\varepsilon$ when this is not misleading.

Remark 2. There is presumably some freedom in the choice of the approximate problem. A natural candidate might have been

$$
Y_{n}:=\left\{u \in H^{1}\left(\Omega_{n}, \mathbb{C}\right): u \equiv 1 \text { on } \partial \Omega_{n}\right\} .
$$

One advantage of $Y_{n}$ is that

$$
p(u)=m(u):=\int_{\Omega_{n}}\left\langle J u, \xi_{1}\right\rangle \quad \text { for all } u \in Y_{n},
$$


which follows easily by integration by parts. Here, $J u$ (the Jacobian of $u$ ) denotes the 2-form on $\Omega_{n}$

$$
J u:=\frac{1}{2} d(u \times d u)=\sum_{i<j}\left(\partial_{i} u \times \partial_{j} u\right) d x_{i} \wedge d x_{j},
$$

and the 2 -form $\xi_{1}$ is defined on $\Omega_{n}$ by

$$
\xi_{1}(x):=\frac{2}{N-1} \sum_{i=2}^{N} x_{i} d x_{1} \wedge d x_{i} .
$$

Finally $\langle\cdot, \cdot\rangle$ stands for the scalar product of 2 -forms. As we will see later, $m$ has a convenient geometric interpretation which we will use throughout. On the torus $\Pi_{n}$, however, $m$ is not well defined (due to $\xi_{1}$ ), and we will have to circumvent this difficulty by choosing suitable unfoldings.

Whereas part of the analysis is somewhat simpler in $Y_{n}$, the main disadvantage is that the translation invariance of our original problem is broken in $Y_{n}$.

Upper bound for $I_{n, \varepsilon}$ and $c_{n, \varepsilon}$. The upper bound on $I_{n, \varepsilon}$ is obtained using appropriate comparison functions for $\left(\mathcal{P}_{n}^{\varepsilon}\right)$. As already mentioned, in the limit $\varepsilon \rightarrow 0$, the solution $u_{\varepsilon}$ (and also $u_{n, \varepsilon}$ ) will ultimately look like thin vortex rings. In what follows, for $R>0$ $(2 R<n)$ we propose a simple construction of such a vortex ring $w_{\varepsilon, R}$ of radius $R$, which will turn out to be an almost optimal candidate.

We carry out the construction in cylindrical coordinates $\left(x_{1}, r, \theta\right)$, where $r:=\left(x_{2}^{2}+\right.$ $\left.\cdots+x_{N}^{2}\right)^{1 / 2}$. The function $w_{\varepsilon, R}$ will be independent of $\theta$ (i.e. cylindrically symmetric); we therefore just need to describe it in the $\left(x_{1}, r\right)$ half-plane $H_{+}$. For that purpose, consider in the complex plane the point $z_{R}:=i R$ and the function $\omega_{R}$ defined on $B_{2 R}$ by

$$
\omega_{R}(z)=\frac{z-z_{R}}{\left|z-z_{r}\right|} \frac{z+z_{R}}{\left|z+z_{r}\right|} \exp (i \varphi),
$$

where $\varphi$ is a real harmonic function such that $\omega_{R} \equiv 1$ on $\partial B_{2 R}$ (see [9]). Then we set

$$
w_{\varepsilon, R}\left(x_{1}, r, \theta\right):= \begin{cases}\omega_{R}\left(x_{1}+i r\right) & \text { if } x_{1}+i r \in B_{2 R} \backslash B_{\varepsilon}\left(z_{R}\right), \\ \varepsilon^{-1}\left|x_{1}+i(r-R)\right| \omega_{R}\left(x_{1}+i r\right) & \text { if } x_{1}+i r \in B_{\varepsilon}\left(z_{R}\right) .\end{cases}
$$

By standard computations,

$$
\begin{aligned}
\frac{1}{2} \int_{\mathbb{R}^{N}}\left|\nabla w_{\varepsilon, R}\right|^{2} & =\frac{1}{2}\left|S^{N-2}\right| \int_{H_{+}}\left|\nabla w_{\varepsilon, R}\right|^{2} r^{N-2} d x_{1} d r \\
& =\pi R^{N-2}\left|S^{N-2}\right||\log \varepsilon|+O(1),
\end{aligned}
$$

and similarly

$$
\frac{1}{4 \varepsilon^{2}} \int_{\mathbb{R}^{N}}\left(1-\left|w_{\varepsilon, R}\right|^{2}\right)^{2}=O(1)
$$

so that

$$
E_{\varepsilon}\left(w_{\varepsilon, R}\right)=\pi\left|S^{N-2}\right| R^{N-2}|\log \varepsilon|+O(1) .
$$


For the momentum $p\left(w_{\varepsilon, R}\right)=m\left(w_{\varepsilon, R}\right)$, we have

$$
\begin{aligned}
p\left(w_{\varepsilon, R}\right) & =\left|S^{N-2}\right| \int_{H_{+}}\left(\frac{\partial w_{\varepsilon, R}}{\partial x_{1}} \times \frac{\partial w_{\varepsilon, R}}{\partial r}\right) \frac{2 r}{N-1} r^{N-2} d x_{1} d r \\
& =2 \pi \frac{\left|S^{N-2}\right|}{N-1} R^{N-1}+o(1)=2 \pi\left|B^{N-1}\right| R^{N-1}+o(1),
\end{aligned}
$$

since

$$
J w_{\varepsilon, R}\left(x_{1}, r, \theta\right)=\left(\partial_{x_{1}} w_{\varepsilon, R} \times \partial_{r} w_{\varepsilon, R}\right) d x_{1} \wedge d r
$$

and since

$$
\partial_{x_{1}} w_{\varepsilon, R} \times \partial_{r} w_{\varepsilon, R} \rightarrow \pi \delta_{(0, R)}
$$

in the sense of measures on $H_{+}$. The detailed computations to obtain estimates (14) and (15) are standard and can be found in many places (see e.g. [9]), so we do not repeat them here. With the help of these estimates, it is then fairly easy to obtain a (sharp) upper bound for $I_{n, \varepsilon}$.

Lemma 1. There exists some constant $K_{0}$, which is independent of $n$ and $\varepsilon$, such that

$$
\left|I_{n, \varepsilon}\right| \leq K_{0}|\log \varepsilon|
$$

Moreover,

$$
\limsup _{\varepsilon \rightarrow 0}\left(\sup _{n \in \mathbb{N}^{*}} \frac{I_{n, \varepsilon}}{|\log \varepsilon|}\right) \leq \pi\left|S^{N-2}\right| .
$$

We next turn to $c_{n, \varepsilon}$. As a consequence of the Pohozaev identity for $(11)$ and some careful analysis of the boundary terms relating $p(u)$ and $m(u)$ in $X_{n}$ we obtain the following.

Lemma 2. There exists $\varepsilon_{1}>0$ such that for $0<\varepsilon<\varepsilon_{1}$ and $n \geq n(\varepsilon)$, where $n(\varepsilon) \in \mathbb{N}$ depends only on $\varepsilon$, we have

$$
\left|c_{n, \varepsilon}\right| \leq K_{1}
$$

Here $K_{1}$ is some constant which is independent of $n$ and $\varepsilon$.

Remark 3. It follows from our proof of Lemma 2 that an upper bound for $n(\varepsilon)$ is $K_{2}|\log \varepsilon| \varepsilon^{3-N}$, where $K_{2}$ is some sufficiently large constant. With a little more work, one should be able to prove that a large (but independent of $\varepsilon$ ) constant is a valid upper bound. Since our final goal is to let $n \rightarrow \infty$ at fixed $\varepsilon$, the first upper bound is sufficient.

\subsection{Some properties of the Euler-Lagrange equation}

An important part of our results relies on the analysis of the Euler-Lagrange equation (11). Since we believe that it is of interest in related topics, as, for instance, superconductivity (see Remark 4(iv)) we will be more general than what is strictly needed for the proof of Theorem 1 . Therefore, we will consider solutions $w_{\varepsilon}$ to the class of equations

$$
i|\log \varepsilon| \mathbf{c}(x) \cdot \nabla w=\Delta w+\frac{1}{\varepsilon^{2}} w\left(1-|w|^{2}\right)-|\log \varepsilon|^{2} d(x) w \quad \text { on } \Omega,
$$


where $\Omega \subseteq \mathbb{R}^{N}$ is a piecewise $\mathcal{C}^{1}$ simply connected domain, $\mathbf{c}: \bar{\Omega} \rightarrow \mathbb{R}^{N}$ is a bounded Lipschitz vector field and $d: \bar{\Omega} \rightarrow \mathbb{R}$ is Lipschitz nonnegative and bounded [in our original problem $\mathbf{c}$ is constant and $d=0$ ]. If we allow $\mathbf{c}$ and $d$ to depend on $\varepsilon$ (in view in particular of the application to superconductivity), then we require that there exists some constant $\Lambda_{0}>0$ not depending on $\varepsilon$ such that

$$
\|\mathbf{c}\|_{L^{\infty}(\Omega)}^{2}+\|\nabla \mathbf{c}\|_{L^{\infty}(\Omega)}^{2}+\|d\|_{L^{\infty}(\Omega)}^{2}+\|\nabla d\|_{L^{\infty}(\Omega)}^{2} \leq \Lambda_{0}^{2} .
$$

Notice that $(19)$ can be rewritten as

$$
i|\log \varepsilon| \mathbf{c}(x) \cdot \nabla w=\Delta w+\frac{1}{\varepsilon^{2}} w\left(a_{\varepsilon}(x)-|w|^{2}\right),
$$

where

$$
a_{\varepsilon}(x):=1-d(x) \varepsilon^{2}|\log \varepsilon|^{2} .
$$

When $\operatorname{div} \mathbf{c} \equiv 0$ it is also equivalent to

$$
\left(\nabla-i|\log \varepsilon| \frac{\mathbf{c}}{2}\right)^{2} w+\frac{1}{\varepsilon^{2}} w\left(b_{\varepsilon}(x)-|w|^{2}\right)=0
$$

where

$$
b_{\varepsilon}(x):=a_{\varepsilon}(x)+\varepsilon^{2}|\log \varepsilon|^{2} \frac{c^{2}(x)}{4} .
$$

Equation (19) is variational when $\mathbf{c}$ is divergence free; we will make this assumption throughout. It is likely however that a large part of the analysis can be done in the general case. Notice also that no boundary condition is prescribed here so that the focus in this section will be on local properties.

The outline of our analysis of (19) follows closely the corresponding theory for the Ginzburg-Landau equation developed in [9, 40, 13, 37, 31, 32, 10, 29, 5, 16] and the references therein. In particular, the emphasis is placed there on the set

$$
S_{\varepsilon}:=\left\{x \in \Omega:\left|w_{\varepsilon}(x)\right| \leq 1 / 2\right\},
$$

where vorticity and energy will eventually concentrate in the limit $\varepsilon \rightarrow 0$. Notice that for the proof of Theorem 1 the structure of $S_{\varepsilon}$ for $\varepsilon$ fixed but expanding $\Omega$ will also play a key role. We first start with the following standard pointwise estimates.

Lemma 3. Let $K$ be any compact subset of $\Omega$. Then, for any solution $w_{\varepsilon}$ of $(19)$ we have:

$$
\left\{\begin{array}{l}
\left\|w_{\varepsilon}\right\|_{L^{\infty}(K)} \leq 1+c_{\infty}^{2} \varepsilon^{2}|\log \varepsilon|^{2}+C \frac{\varepsilon^{2}}{\operatorname{dist}(K, \partial \Omega)^{2}}, \\
\left\|\nabla w_{\varepsilon}\right\|_{L^{\infty}(K)} \leq C_{K} / \varepsilon
\end{array}\right.
$$

where $c_{\infty}:=\|\mathbf{c}\|_{L^{\infty}}, C$ is a constant depending only on $N$, and $C_{K}$ is a constant depending only on $N, c_{\infty}$ and $K$. 
In order to describe the properties of $S_{\varepsilon}$, monotonicity formulas play an important role (as in the works quoted above). More generally, they have been extensively used in the context of regularity for various problems in PDE's and geometry (see e.g. [34, 23]).

For $x_{0} \in \Omega$ and $r>0$ such that $B_{r}\left(x_{0}\right) \subset \Omega$, consider the scaled energy

$$
\begin{aligned}
\tilde{E}_{\varepsilon}\left(w_{\varepsilon}, x_{0}, r\right) & :=\frac{1}{r^{N-2}} E_{\varepsilon}\left(w_{\varepsilon}, x_{0}, r\right) \\
& \equiv \frac{1}{r^{N-2}} \int_{B_{r}\left(x_{0}\right)}\left(\frac{\left|\nabla w_{\varepsilon}\right|^{2}}{2}+\frac{\left(a_{\varepsilon}(x)-\left|w_{\varepsilon}\right|^{2}\right)^{2}}{4 \varepsilon^{2}}\right)
\end{aligned}
$$

When this does not lead to confusion, we will also denote it by $\tilde{E}_{\varepsilon}\left(x_{0}, r\right)$ or even $\tilde{E}_{\varepsilon}(r)$.

Lemma 4. There exists $C>0$ depending only on $N$ such that for

$$
\Lambda:=C\left(c_{\infty}+1\right)|\log \varepsilon|, \quad Q:=C \Lambda_{0}|\log \varepsilon|^{2} \varepsilon,
$$

and for any $w_{\varepsilon}$ satisfying 19$]$ on $B_{R}\left(x_{0}\right) \subset \Omega$, we have

$$
\begin{aligned}
\frac{d}{d r}\left(\exp (\Lambda r)\left(\tilde{E}_{\varepsilon}\left(x_{0}, r\right)+\frac{Q^{2}}{\Lambda}\right)\right) \geq & \frac{1}{r^{N-2}} \int_{\partial B_{r}}\left|\frac{\partial w_{\varepsilon}}{\partial n}\right|^{2} \\
& +\frac{1}{r^{N-1}} \int_{B_{r}} \frac{\left(a_{\varepsilon}(x)-\left|w_{\varepsilon}\right|^{2}\right)^{2}}{4 \varepsilon^{2}} \geq 0
\end{aligned}
$$

for $0<r<R$. In particular, $\exp (\Lambda r)\left(\tilde{E}_{\varepsilon}\left(x_{0}, r\right)+Q^{2} / \Lambda\right)$ is increasing.

The above inequality is obtained using a crude estimate for the Jacobian $J w_{\varepsilon}$. This restricts somehow its usefulness to balls of size $O(1 /|\log \varepsilon|)$. In order to handle balls of radius $O(1)$, refined estimates on Jacobian integrals are needed (see [29, 1]).

Proposition 2. There exist $C>0$ and $\beta>0$ depending only on $N$ such that for any $w_{\varepsilon}$ satisfying (19) on $B_{R}\left(x_{0}\right) \subset \Omega$, we have

$$
\tilde{E}_{\varepsilon}\left(x_{0}, \theta r\right) \leq C\left(\tilde{E}_{\varepsilon}\left(x_{0}, r\right)+\left(1+\Lambda_{0}\right)^{N-1} \varepsilon^{\beta}\right)
$$

for $0<\theta<1 / 2$ and $0<r<\min \left(R, 2 /\left(\Lambda_{0}+1\right)\right)$

Using the previous two results, and following the arguments of [10] (see also [37, 31, 32]), we derive the following result, which plays an important role in the analysis.

Theorem 2. Let $w_{\varepsilon}$ be a solution of (19) on $\Omega$ and $\sigma>0$ be given. There exist constants $\eta>0$ and $\varepsilon_{0}>0$, depending only on $N, \sigma$ and $\Lambda_{0}$, such that if $x_{0} \in \Omega, \varepsilon \leq \varepsilon_{0}$, $\sqrt{\varepsilon} \leq r \leq 1 /\left(1+\Lambda_{0}\right), B_{2 r}\left(x_{0}\right) \subset \Omega$, and

$$
\tilde{E}_{\varepsilon}\left(x_{0}, r\right) \leq \eta|\log \varepsilon|,
$$

then

$$
\left|w_{\varepsilon}\left(x_{0}\right)\right| \geq 1-\sigma .
$$


Asymptotic analysis of concentrating measures. We assume from now on that $w_{\varepsilon}$ satisfies the bound

$$
E_{\varepsilon}\left(w_{\varepsilon}\right)=\int_{\Omega} e_{\varepsilon}\left(w_{\varepsilon}\right) \leq M_{0}|\log \varepsilon|,
$$

where $M_{0}$ is some fixed constant. In this regime, one of the main consequences of Theorem 2 is that as $\varepsilon$ tends to zero, the set $S_{\varepsilon}$ concentrates on a rectifiable limiting set $S_{*}$, of locally finite $N-2$ Hausdorff measure. It is convenient to introduce the following measures:

$$
\left\{\begin{array}{l}
\mu_{\varepsilon}:=\frac{e_{\varepsilon}\left(w_{\varepsilon}\right)}{|\log \varepsilon|} d x, \\
\eta_{\varepsilon}:=\varepsilon^{-2} 1_{S_{\varepsilon}} d x, \\
J_{\varepsilon}:=J w_{\varepsilon} .
\end{array}\right.
$$

In view of assumption (24), $\mu_{\varepsilon}$ is bounded. Therefore, up to a subsequence we may assume that

$$
\mu_{\varepsilon} \rightarrow \mu_{*} \quad \text { as measures. }
$$

Using Theorem 2 again, combined with a Besicovitch covering argument, we find that $\varepsilon^{-2} 1_{S_{\varepsilon}}$ is locally bounded in $L^{1}(\Omega)$. Extracting possibly a further subsequence, we may thus assume that

$$
\eta_{\varepsilon} \rightarrow \eta_{*} \quad \text { as measures. }
$$

Concerning $J_{\varepsilon}$ (a measure with values in 2-forms), it is tempting to believe that it is also bounded in $L_{\text {loc }}^{1}(\Omega)$. We have no proof of that fact, but we may invoke Jerrard-Soner's [29] compactness result (valid for arbitrary functions satisfying (24), see also [1]) to assert that $J_{\varepsilon}$ is bounded in $\left[\mathcal{C}^{0, \alpha}(K)\right]^{*}$ for any compact $K \subset \Omega$ and any $0<\alpha<1$. Going possibly to a third subsequence, we thus have

$$
J_{\varepsilon} \rightarrow J_{*} \quad \text { in }\left[\mathcal{C}^{0, \alpha}\left(K, \Lambda_{2} \mathbb{R}^{N}\right)\right]^{*}, \quad \text { for every compact } K \subset \Omega .
$$

It is proved moreover in [29, 1] that

$$
\left\|J_{*}\right\| \leq \mu_{*},
$$

and that the current $\llbracket J_{*} \rrbracket$ associated to $J_{*}$ is an integer multiplicity $(N-2)$-current. In particular, its geometrical support

$$
\Sigma_{J}:=\left\{x \in \Omega: \Theta_{N-2}\left(\left\|J_{*}\right\|, x\right)>0\right\}
$$

is an $(N-2)$-rectifiable set. Here, for a Radon measure $v \in \mathcal{M}(\Omega)$ and $m>0$, the $m$-dimensional density of $v$ at $x \in \Omega$ is defined by

$$
\Theta_{m}(\nu, x):=\liminf _{r \rightarrow 0} \frac{v(B(x, r))}{r^{m}} .
$$

Likewise we set

$$
\Sigma_{\mu}=\left\{x \in \Omega: \Theta_{N-2}\left(\mu_{*}, x\right)>0\right\}
$$

and similarly we define $\Sigma_{\eta}$.

In the next theorem we will clarify the structure of the measure $\mu_{*}$ and we will specify its relation to $J_{*}$. We emphasize that no boundary condition has been prescribed on $\partial \Omega$. 
Theorem 3. The following properties hold.

(i) The set $\Sigma_{\mu}$ is closed in $\Omega$ and $(N-2)$-rectifiable. There exists $\eta_{0}>0$ such that for each $x_{0} \in \Sigma_{\mu}$,

$$
\Theta_{*}\left(x_{0}\right):=\Theta_{N-2}\left(\mu_{*}, x_{0}\right)=\liminf _{r \rightarrow 0} \frac{\mu_{*}\left(B_{r}\left(x_{0}\right)\right)}{r^{N-2}} \geq \eta_{0} .
$$

Moreover, for every compact set $F \subset \Omega \backslash \Sigma_{\mu}$,

$$
\left|w_{\varepsilon}(x)\right| \rightarrow 1 \quad \text { uniformly on } F \text { as } \varepsilon \rightarrow 0 .
$$

(ii) The measure $\mu_{*}$ can be decomposed as

$$
\mu_{*}=|\nabla h(x)|^{2} \cdot \mathcal{H}^{N}+\Theta_{*}(x) \cdot \mathcal{H}^{N-2}\left\llcorner\Sigma_{\mu},\right.
$$

where $h$ is some harmonic function.

(iii) Let $K \subset \Omega$ be any compact set. There exists some constant $C_{K}$, depending only on $K$, such that

$$
\left(C_{K}\right)^{-1} J_{*} \leq \eta_{*} \leq C_{K} M_{0} \mu_{*} .
$$

(iv) The varifold $V:=V\left(\Sigma_{\mu}, \Theta_{*}\right)$ satisfies the equation

$$
\mathbf{H}(x)=*\left(\mathbf{c}(x) \wedge * \frac{d J_{*}}{d \mu_{*}}\right) \quad \text { for } \mu_{*} \text {-a.e. } x \text { in } \Sigma_{\mu},
$$

where $\mathbf{H}(x)$ denotes the generalized mean curvature of $V$ at $x, *$ refers to the Hodge duality, and $d J_{*} / d \mu_{*}$ is the Radon-Nikodym derivative of $J_{*}$ with respect to $\mu_{*}$.

A short comment is needed concerning the interpretation of (29). The generalized mean curvature $\mathbf{H}$ of the varifold $V$ is defined by (see [39])

$$
\int_{\Omega} \operatorname{div}_{\Sigma_{\mu}} \mathbf{X}=-\int_{\Omega} \mathbf{H} \cdot \mathbf{X} \quad \text { for all } \mathbf{X} \in \mathcal{C}_{\mathrm{c}}^{\infty}\left(\Omega, \mathbb{R}^{N}\right),
$$

where $\operatorname{div}_{\Sigma_{\mu}}$ denotes the divergence restricted to the tangent space. Moreover, we identify vector fields and 1 -forms.

Remark 4. (i) In the case $\mathbf{c} \equiv 0$ and $d \equiv 0,(19)$ is the standard Ginzburg-Landau equation and then (29) means that $V$ is a stationary varifold (see [5, 10]).

(ii) Equation (29) is very reminiscent of the prescribed mean curvature equation in codimension 1 . However here, in codimension 2, an important difference is that the right hand side of (29) does depend on $V$ through its tangent space. To give a flavor of the structure of (29), let us first consider the case $N=3$, and $d\left\|J_{*}\right\| / d \mu_{*}=1$. Then $V$ is a smooth curve and (29)

$$
\kappa=\mathbf{c} \times \tau,
$$

where $\tau$ is the unit tangent vector to $V$ and $\kappa$ its curvature vector. In the case $\mathbf{c} \equiv \mathbf{c}_{0}$ is a constant vector field, the solutions are

- straight lines parallel to $\mathbf{c}_{0}$,

- circles of radius $1 / c_{0}$ in a plane orthogonal to $\mathbf{c}_{0}$,

- helicoids of axis parallel to $\mathbf{c}_{0}$. 
On the other hand, any constant mean curvature hypersurface in dimension $N-1$ yields a solution of (29) for some constant vector field $\mathbf{c}_{0}$. In dimension 3 , this yields, as already mentioned, the round circle as unique compact solution. In higher dimensions, however, there is a rich class of constant mean curvature hypersurfaces besides spheres (e.g. Wente's tori in dimension $N=4$ ). It is tempting to believe that any compact solution of 29 with a constant vector field $\mathbf{c}_{0}$ is contained in an affine hyperplane (and is thus a constant mean curvature hypersurface).

(iii) In the case $d\left\|J_{*}\right\| / d \mu_{*}=1, V$ has integer multiplicity. In the optimal case where $J_{*}$ has constant multiplicity, it follows from [29] and Allard's theorem (see [2, 39]) that $V$ is a $\mathcal{C}^{1, \alpha}$ manifold.

(iv) Equation 19 with $\mathbf{c}(x)=A(x)$ and $d(x)=|A(x)|^{2} / 4$ is the first equation in the Ginzburg-Landau system of superconductivity, namely

$$
(\nabla-i A|\log \varepsilon| / 2)^{2} u=\frac{1}{\varepsilon^{2}} u\left(1-|u|^{2}\right) .
$$

In particular, for solutions satisfying the energy bound 24 in the Coulomb gauge, vortices will be curved according to the equation

$$
\kappa=A \times \tau,
$$

provided $d\left\|J_{*}\right\| / d \mu_{*}=1$.

Theorem 3 states some compactness properties for the measures. However, without assumptions on the boundary data, we cannot expect compactness for the functions $w_{\varepsilon}$, as noticed in [17]. The presence in the decomposition (28) of one part which is absolutely continuous with respect to the Lebesgue measure is precisely due to possible wild oscillations of $w_{\varepsilon}$ on the boundary.

Asymptotics for $w_{\varepsilon}$. If we impose boundary conditions on $\partial \Omega$, then we may obtain compactness properties for the sequence $w_{\varepsilon}$. In this subsection, we will focus only on the case which is of interest for Theorem 1 , namely

$$
\Omega:=\Pi_{n} \simeq \Omega_{n},
$$

with the convention that $\Pi_{\infty}:=\mathbb{R}^{N}$; we refer to Appendix A for more general statements. We make the assumption that

$$
n \geq\left(M_{0}+1\right)|\log \varepsilon|
$$

The main point here is that we would like to obtain estimates which are uniform with respect to the domain size (i.e. independent of $n$ ). In this situation we obtain:

Theorem 4. Let $w_{\varepsilon}$ be a solution of (11) such that (24) and (31) are satisfied.

(i) Let $1 \leq p<N /(N-1)$. Then there exists some constant $C$, depending only on $p$, $\Lambda_{0}$ and $M_{0}$, but independent of $\varepsilon$ and $n$, such that for any $x_{0} \in \Pi_{n}$ we have

$$
\int_{B\left(x_{0}, 1\right)}\left|\nabla w_{\varepsilon}\right|^{p} \leq C .
$$


(ii) There exist $R>0, C>0$ and $l \in \mathbb{N}$, depending only on $\Lambda_{0}$ and $M_{0}$, and $q$ points $x_{1, \varepsilon}, \ldots, x_{q, \varepsilon}(q \leq l)$ in $\Pi_{n}$ such that $S_{\varepsilon} \subset \bigcup_{i=1}^{q} B\left(x_{i, \varepsilon}, R\right), B\left(x_{i, \varepsilon}, 8 R\right) \cap$ $B\left(x_{j, \varepsilon}, 8 R\right)=\emptyset$ if $i \neq j$, and

$$
\int_{\Pi_{n} \backslash \cup B\left(x_{i, \varepsilon}, R\right)} e_{\varepsilon}\left(w_{\varepsilon}\right) \leq C .
$$

\subsection{The isoperimetric problem}

After this rather lengthy discussion on the Euler-Lagrange equation, we go back to our original problem and consider from now on only minimizers $u_{n, \varepsilon}$ of $\left(\mathcal{P}_{n}^{\varepsilon}\right)$. Since our ultimate goal is to provide the existence of a solution $u_{\varepsilon}$ of (11) as well as some qualitative properties (see Theorem 1), we will eventually let $n$ go to $\infty$ keeping $\varepsilon$ fixed (in particular, we assume throughout that (31) is satisfied). In order to describe properly the behavior of $u_{\varepsilon}$ (including the stability properties, which will be discussed later), it is extremely important, in this approach, to get more information than a simple $H_{\text {loc }}^{1}$ convergence.

The first crucial observation in this section is the relation of the energy $E_{\varepsilon}\left(u_{n, \varepsilon}\right)$ and the flux $p\left(u_{n, \varepsilon}\right)$ with geometrical properties of $J u_{n, \varepsilon}$ (as already observed in [14] and [28]). This relation is best understood by taking the limit as $\varepsilon$ tends to 0 when $n$ is fixed (note, however, that this is incompatible with $n \geq n(\varepsilon)$ of Lemma 2). It follows from the analysis of [29, 1] that, up to a subsequence,

$$
J u_{n, \varepsilon} \rightarrow \pi T_{n}=\pi \partial R_{n} \quad \text { in }\left[\mathcal{C}^{0,1}\left(\Pi_{n}\right)\right]^{*},
$$

where $T_{n}=\partial R_{n}$ is an $(N-2)$-dimensional integral boundary, i.e. $T_{n}$ is a rectifiable current with integer multiplicities (of course the choice of the rectifiable current $R_{n}$ is not unique). Moreover,

$$
p\left(u_{n, \varepsilon}\right) \rightarrow \pi \partial R_{n}\left(* \xi_{1}\right)=\pi R_{n}\left(* d^{*} \xi_{1}\right)=2 \pi R_{n}\left(* d x_{1}\right) \equiv \mathcal{F}\left(T_{n}\right),
$$

where $\mathcal{F}\left(T_{n}\right)$ represents $2 \pi$ times the flux of the vector $\mathbf{e}_{1}$ through $R_{n}$. Notice that in particular $\mathcal{F}\left(T_{n}\right) \leq 2 \pi \mathbf{M}\left(R_{n}\right)$. On the other hand, it is also proved in [29, 1] that

$$
\liminf _{\varepsilon \rightarrow 0} \frac{E_{\varepsilon}\left(u_{n, \varepsilon}\right)}{\pi|\log \varepsilon|} \geq \mathbf{M}\left(T_{n}\right) .
$$

This establishes immediately the inequality

$$
\frac{\mathbf{M}\left(\partial R_{n}\right)^{\frac{N-1}{N-2}}}{\mathbf{M}\left(R_{n}\right)} \leq \liminf _{\varepsilon \rightarrow 0} \frac{2 \pi E_{\varepsilon}\left(u_{n, \varepsilon}\right)^{\frac{N-1}{N-2}}}{(\pi|\log \varepsilon|)^{\frac{N-1}{N-2}} p\left(u_{n, \varepsilon}\right)} .
$$

Using Lemma 1 we deduce that

$$
\frac{\mathbf{M}\left(\partial R_{n}\right)^{\frac{N-1}{N-2}}}{\mathbf{M}\left(R_{n}\right)} \leq \lambda_{N} \equiv \frac{\left|S^{N-2}\right|^{\frac{N-1}{N-2}}}{\left|B^{N-1}\right|} .
$$

Since the right hand side of 33 is the best constant in the isoperimetric inequality it follows that $T_{n}=\partial R_{n}$ is a round $(N-2)$-sphere (contained in an $(N-1)$-hyperplane orthogonal to $\mathbf{e}_{1}$ ). 
In our situation, we will obtain an inequality similar to (33), but uniformly for $n$ large. To be more precise, assume from now on that $n \geq n(\varepsilon)$, where $n(\varepsilon)$ was defined in Lemma 2, Then we have

Lemma 5. For every $n \geq n(\varepsilon)$ there exists an $(N-2)$-dimensional integral boundary $T_{n, \varepsilon}=\partial R_{n, \varepsilon}$ supported in at most $\ell$ balls of radius $R(\ell, R$ being independent of $n$ and $\varepsilon)$, such that

(i) $\left\|J u_{n, \varepsilon}-\pi T_{n, \varepsilon}\right\|_{\left[\mathcal{C}^{0,1}\left(\Pi_{n}\right)\right]^{*}} \leq r(\varepsilon)$,

(ii) $\left|p\left(u_{n, \varepsilon}\right)-\mathcal{F}\left(T_{n, \varepsilon}\right)\right| \leq r(\varepsilon)$,

(iii) $\mathbf{M}\left(T_{n, \varepsilon}\right) \leq \frac{E_{\varepsilon}\left(u_{n, \varepsilon}\right)}{\pi|\log \varepsilon|}+r(\varepsilon)$,

where $r(\varepsilon) \rightarrow 0$ as $\varepsilon \rightarrow 0$, independently of $n$.

As mentioned, the choice of a current $R_{n, \varepsilon}$ such that $T_{n, \varepsilon}=\partial R_{n, \varepsilon}$ is not unique. We may therefore additionally require that

$$
\mathbf{M}\left(R_{n, \varepsilon}\right)=\inf \left\{\mathbf{M}(R): \partial R=T_{n, \varepsilon}\right\} .
$$

For such a choice (which is always possible by [20,4.1.12]), the following isoperimetric inequality is valid (see [3]):

$$
\frac{\mathbf{M}\left(T_{n, \varepsilon}\right)^{\frac{N-1}{N-2}}}{\mathbf{M}\left(R_{n, \varepsilon}\right)} \geq \lambda_{N} .
$$

Proposition 3. We have

$$
\frac{\mathbf{M}\left(\partial R_{n, \varepsilon}\right)^{\frac{N-1}{N-2}}}{\mathbf{M}\left(R_{n, \varepsilon}\right)}=\lambda_{N}+r(\varepsilon),
$$

where $r(\varepsilon) \rightarrow 0$ as $\varepsilon \rightarrow 0$, independently of $n$. In particular, for all sequences $\varepsilon_{j} \rightarrow 0$ and $n_{j} \geq n\left(\varepsilon_{j}\right)$ there exist subsequences (still denoted $\varepsilon_{j}$ and $n_{j}$ ) and translations $\tau_{j}$ in $\Pi_{n_{j}}$ such that

$$
\tau_{j} T_{n_{j}, \varepsilon_{j}} \rightarrow S^{N-2} \quad \text { in }\left[\mathcal{C}_{\mathrm{c}}^{0,1}\left(\mathbb{R}^{N}\right)\right]^{*} \text { as } j \rightarrow \infty,
$$

where $S^{N-2}$ is the unit round $(N-2)$-sphere contained in the hyperspace orthogonal to $\mathbf{e}_{1}$.

Remark 5. Actually, as $j \rightarrow \infty$ we have $\tau_{j} R_{n_{j}, \varepsilon_{j}} \rightarrow B^{N-1}$ and also $\tau_{j} T_{n_{j}, \varepsilon_{j}} \rightarrow S^{N-2}$ in the flat norm sense (see [20, 4.1.12]), with $\mathbf{M}\left(R_{n_{j}, \varepsilon_{j}}\right) \rightarrow\left|B^{N-1}\right|$ and $\mathbf{M}\left(T_{n_{j}, \varepsilon_{j}}\right) \rightarrow$ $\left|S^{N-2}\right|$.

Note that 37) states a rather weak convergence. In particular, it does not exclude very small structures even far from the limit $S^{N-2}$. The next lemma, which improves statement (ii) of Theorem 4, excludes such structures.

Lemma 6. There exist $R>0, C>0$ independent of $\varepsilon$ and $n$, and $x_{n, \varepsilon} \in \Pi_{n}$ such that

(i) $S_{\varepsilon}\left(u_{n, \varepsilon}\right) \subset B\left(x_{n, \varepsilon}, R\right)$,

(ii) $\int_{\Pi_{n} \backslash B\left(x_{n, \varepsilon}, R\right)} e_{\varepsilon}\left(u_{n, \varepsilon}\right) \leq C$,

for every $\varepsilon \leq \varepsilon_{0}$ and $n \geq n(\varepsilon)$, $\varepsilon_{0}$ being independent of $n$. 
As already mentioned, our problem is invariant under translation. We now remove this invariance. To that end, in view of Lemma 6 and Proposition 3 , we assume that the identification $\Pi_{n} \simeq[-n, n]^{N}$ is such that

$$
x_{n, \varepsilon}=0 \quad \text { and } \quad J u_{n_{j}, \varepsilon_{j}} \rightarrow \pi S^{N-2} \quad \text { in }\left[\mathcal{C}_{\mathrm{c}}^{0,1}\left(\mathbb{R}^{N}\right)\right]^{*}
$$

for all sequences $\varepsilon_{j} \rightarrow 0$ and $n_{j} \geq n\left(\varepsilon_{j}\right)$, where $S^{N-2}$ is the unit $(N-2)$-sphere contained in the subspace orthogonal to $\mathbf{e}_{1}$.

\subsection{Limits of growing tori}

It remains at this stage, for fixed $\varepsilon$ (but chosen sufficiently small), to let $n \rightarrow \infty$. Since $E_{\varepsilon}\left(u_{n, \varepsilon}\right)$ is bounded uniformly in $n$ by Lemma 1 (but not in $\varepsilon$ !), up to a possible subsequence we may assume

$$
u_{n, \varepsilon} \rightarrow u_{\varepsilon} \quad \text { in } H_{\mathrm{loc}}^{1}\left(\mathbb{R}^{N}\right) \text { as } n \rightarrow \infty,
$$

so that

$$
E_{\varepsilon}\left(u_{\varepsilon}\right) \leq \liminf _{n \rightarrow \infty} E_{\varepsilon}\left(u_{n, \varepsilon}\right) .
$$

Moreover, by standard elliptic estimates ( $\varepsilon$ is fixed),

$$
u_{n, \varepsilon} \rightarrow u_{\varepsilon} \quad \text { strongly in } H_{\text {loc }}^{1}\left(\mathbb{R}^{N}\right) \quad \text { as } n \rightarrow \infty .
$$

Note also that since $\left(u_{n, \varepsilon}\right)_{n \in \mathbb{N}}$ is bounded in $L^{\infty}$, so is $u_{\varepsilon}$, and we may pass to the limit in the equation. Hence, $u_{\varepsilon}$ satisfies (11) with

$$
c(\varepsilon)=\lim _{n \rightarrow \infty} c_{n, \varepsilon} .
$$

Since we also have $J u_{n, \varepsilon} \rightarrow \pi S^{N-2}$ as $\varepsilon \rightarrow 0$, for fixed but small $\varepsilon$ the Jacobian $J u_{n, \varepsilon}$ is far from zero (for all $n \geq n(\varepsilon)$ ) and therefore $u_{\varepsilon}$ is not a trivial solution. Hence, existence of $U_{\varepsilon}(x):=u_{\varepsilon}(\varepsilon x)$ in Theorem 1 is established. Properties (6)-(9) then follow from the analysis of Subsection 1.3 (see Section 4 for the details).

The definition of $P\left(U_{\varepsilon}\right)$ needs some clarification. For this purpose, we consider the class of functions

$$
W=\left\{u \in L^{\infty}\left(\mathbb{R}^{N}\right): E_{\varepsilon}(u)<\infty \text { and } \exists R>0: \inf _{|x| \geq R}|u(x)| \geq 1 / 2\right\} .
$$

If $u \in W$, we may write, for $|x|>R$,

$$
u=\rho \exp i \varphi
$$

where $\varphi$ is a real function on $\mathbb{R}^{N} \backslash B_{R}(0)$ defined modulo a multiple of $2 \pi$. We define

$$
p(u):=\int_{\mathbb{R}^{N}}\left(i u, \partial_{1} u\right) \chi+\int_{\mathbb{R}^{N}}(1-\chi)\left(\rho^{2}-1\right) \partial_{1} \varphi+\int_{\mathbb{R}^{N}} \varphi \partial_{1}(1-\chi),
$$

where $\chi$ is an arbitrary smooth function with compact support such that $\chi \equiv 1$ on $B_{R}(0)$ and $0 \leq \chi \leq 1$. One checks immediately that the definition makes sense in $W$ and is 
independent of the choice of $\chi$ and $\varphi$. [To motivate this choice, notice that formally

$$
\begin{aligned}
\int_{\mathbb{R}^{N}}\left(i u, \partial_{1} u\right) & =\int_{\mathbb{R}^{N}}\left(i u, \partial_{1} u\right) \chi+\int_{\mathbb{R}^{N}}\left(i u, \partial_{1} u\right)(1-\chi) \\
& =\int_{\mathbb{R}^{N}}\left(i u, \partial_{1} u\right) \chi+\int_{\mathbb{R}^{N}}(1-\chi) \rho^{2} \partial_{1} \varphi \\
& =\int_{\mathbb{R}^{N}}\left(i u, \partial_{1} u\right) \chi+\int_{\mathbb{R}^{N}}(1-\chi)\left(\rho^{2}-1\right) \partial_{1} \varphi+\int_{\mathbb{R}^{N}} \varphi \partial_{1}(1-\chi)
\end{aligned}
$$

so that we recover the usual formula when $\nabla u \in L^{1}\left(\mathbb{R}^{N}\right)$.] Clearly, in view of our analysis, $u_{\varepsilon} \in W$ so that $P\left(U_{\varepsilon}\right):=\varepsilon^{N-1} p\left(u_{\varepsilon}\right)$ is well defined.

Remark 6. Consider the affine space

$$
Y=H^{1}\left(\mathbb{R}^{N}\right)+\{1\}=\left\{u=1+v: v \in L^{2}\left(\mathbb{R}^{N}\right), \nabla v \in L^{2}\left(\mathbb{R}^{N}\right)\right\},
$$

equipped with the $H^{1}$-distance. For functions in $Y$, one may set

$$
\tilde{p}_{1}(u)=\int_{\mathbb{R}^{N}}\left(u-1, \frac{\partial u}{\partial x_{1}}\right)
$$

as a definition of the momentum. It is straightforward to see that $\tilde{p}_{1}$ is continuous on $Y$ (for the $H^{1}$-norm). On the other hand, $\mathcal{C}_{\mathrm{c}}^{\infty}\left(\mathbb{R}^{N}\right)+\{1\}$ is dense in $Y$, and included in $W$. One verifies, in view of the definition of $p$, that

$$
\tilde{p}_{1}(u)=p_{1}(u), \quad \forall u \in \mathcal{C}_{\mathrm{c}}^{\infty}\left(\mathbb{R}^{N}\right)+\{1\} \subset W .
$$

\subsection{Discussion on stability}

The discussion about stability of special solutions for dynamical systems is a fundamental issue, in particular if one aims at some physical relevance. This is a vast topic, and the very notion of stability appears in different places with different meanings. We want to stress first that we are not yet able to state any trully satisfactory result concerning the stability of $U_{\varepsilon}$. We next explain the main difficulty in this direction, and the partial results we have obtained.

When dealing with PDE's, a first step commonly needed for stability is to solve the Cauchy problem, at least in a neighborhood of the special solution. In particular, one has to define a suitable function space, and this usually requires some knowledge of the decay properties of the solution. In our case, it can be proved (see [14]) that the Cauchy problem is well defined on $Y=H^{1}\left(\mathbb{R}^{N}\right) \cap L^{4}\left(\mathbb{R}^{N}\right)+\{1\}$ and that both energy $E_{\varepsilon}$ and momentum $\tilde{p}$ are conserved during the flow. However, it is not known whether the solution $u_{\varepsilon}$ belongs to $Y$ (see however results by Gravejat [25] for the asymptotic behavior of finite energy traveling waves), and the possibility to solve the Cauchy problem in other spaces has not been investigated yet.

Assume that in some way one is able to overcome this difficulty. Then in our context the notion of (nonlinear) orbital stability seems to be the most suitable (see e.g. [6, 15, 18, 26]). Indeed, recall that our solution is obtained as a limit of constrained minimizers 
for which both the constraint and the minimized quantity are conserved by the flow. We will show that $u_{\varepsilon}$ is itself a constrained minimizer. For this purpose, set

$$
\Gamma_{\infty}:=\left\{u \in W: p(u)=2 \pi\left|B^{N-1}\right|\right\} .
$$

Theorem 5. We have

$$
p\left(u_{\varepsilon}\right)=2 \pi\left|B^{N-1}\right|
$$

so that $u_{\varepsilon} \in W$ and

$$
E_{\varepsilon}\left(u_{\varepsilon}\right):=\inf _{u \in \Gamma_{\infty}} E_{\varepsilon}(u)
$$

The proof of Theorem 5 relies essentially on the following proposition which provides a decay of the energy at infinity.

Proposition 4. There exist constants $\lambda>0$ and $C>0$, independent of $n \geq n(\varepsilon)$, such that

$$
\int_{\Omega_{n} \backslash B(R)} e_{\varepsilon}\left(u_{n, \varepsilon}\right) \leq C R^{-\lambda} .
$$

In particular,

$$
\lim _{n \rightarrow \infty} E_{\varepsilon}\left(u_{n, \varepsilon}\right)=E_{\varepsilon}\left(u_{\varepsilon}\right)
$$

and

$$
\lim _{n \rightarrow \infty} p\left(u_{n, \varepsilon}\right)=p\left(u_{\varepsilon}\right)=2 \pi\left|B^{N-1}\right| .
$$

Recall that the definition of $p\left(u_{\varepsilon}\right)$ was given in 38 .

Remark 7. (i) The result of Proposition 4 is an exact result for fixed $\varepsilon$, and has to be compared with the weaker asymptotic result

$$
E_{\varepsilon}\left(u_{n, \varepsilon}\right)=E_{\varepsilon}\left(u_{\varepsilon}\right)+O(1) \quad \text { as } \varepsilon \rightarrow 0,
$$

which is an easy consequence of Theorems 1 and 4

(ii) In fact, it follows from the proof of Proposition 4 that 41 holds for any $\lambda<$ $\sqrt{N-1}$, provided $\varepsilon$ is sufficiently small. One might expect, however, that the gradient of $u_{\varepsilon}$ decays as the gradient of $U_{*}$, and $\lambda=N$ should be the optimal constant in (41).

(iii) The statements in Proposition 4 essentially mean that there is no loss of compactness at infinity (it excludes for example a sliding bump "escaping" towards infinity, or vanishing but widespread oscillations).

Comments. (i) The existence of a unique solution for the Cauchy problem in $H^{1}\left(\Pi_{n}\right)$ is standard. Moreover, it is easily proved that the set of minimizers for $\left(\mathcal{P}_{n, \varepsilon}\right)$ (which contains $u_{n, \varepsilon}$ ) is orbitally stable. In particular, the uniqueness of $u_{n, \varepsilon}$ (up to translation and multiplication by a complex number of modulus one) would imply its orbital stability.

(ii) One may wonder whether there is a direct proof (i.e. avoiding the approximate problems) of Theorem 5, and thus also of Theorem 1 . This seems to be a difficult task, mainly since $W$ is not open. 
(iii) A rigorous proof of the orbital stability of $U_{\varepsilon}$ would require, in addition to solving the Cauchy problem, obtaining compactness properties for minimizing sequences for 40 . We will not tackle this problem here.

Added in proof. After the completion of this work, P. Gravejat was able to prove that any finite energy solution (in particular $u_{\varepsilon}$ ) belongs to $Y$. It follows therefore from Remark 6 that

$$
E_{\varepsilon}\left(u_{\varepsilon}\right)=\inf \left\{E_{\varepsilon}(u): u \in Y, \tilde{p}(u)=2 \pi\left|B^{N-1}\right|\right\},
$$

which is certainly an important step towards orbital stability, since, as mentioned, the Cauchy problem is well defined on $Y$.

\subsection{Cylindrically symmetric solutions}

Since equation (11) is invariant under rotations preserving the $x_{1}$ axis, it is tempting to believe that up to a translation $U_{\varepsilon}$ inherits this symmetry; i.e. that $U_{\varepsilon}\left(x_{1}, x^{\prime}\right)$ depends only on $x_{1}$ and $\left|x^{\prime}\right|$, where $x^{\prime}=\left(x_{2}, \ldots, x_{N}\right)$. We have no proof of this fact. However, the following variant of Theorem 1 can be easily established with minor changes in the proof.

Theorem 6. There exists $\varepsilon_{1}>0$ such that for every $0<\varepsilon<\varepsilon_{1}$ there is a solution $\mathcal{U}_{\varepsilon}$ to equation (5) with $C=C(\varepsilon)$ satisfying (6)-(9) and such that $\mathcal{U}_{\varepsilon}$ is cylindrically symmetric.

The slight change is to introduce the space $Z_{n}$ of axially symmetric functions on $[-n, n]^{N}$ with periodic boundary conditions:

$$
\begin{aligned}
Z_{n}:=\left\{u \in H^{1}\left([-n, n]^{N}\right): u=u\left(x_{1},\left|x^{\prime}\right|\right) \text { and } \forall k\right. & \in\{1, \ldots, N\} \\
u\left(x_{1}, \ldots, x_{k-1},-n, x_{k+1}, \ldots, x_{N}\right) & \left.=u\left(x_{1}, \ldots, x_{k-1}, n, x_{k+1}, \ldots, x_{N}\right)\right\}
\end{aligned}
$$

and to consider the minimization problem

$$
\inf \left\{E_{\varepsilon}(u): u \in Z_{n}, p(u)=2 \pi\left|B^{N-1}\right|\right\} .
$$

All the arguments in the proof of Theorem 1 can be carried out similarly if we work with $Z_{n}$ instead of $X_{n}$, yielding the proof of Theorem 6 .

We emphasize, however, an important difference, concerning stability. Stability properties of $\mathcal{U}_{\varepsilon}$ can be obtained (in the same way) for axially symmetric perturbations only. This is a rather restricted class, and it seems difficult to obtain stability results for general perturbations.

Remark 8. (i) As already mentioned, we nevertheless suspect that, up to translation and multiplication by a complex number of modulus one, $U_{\varepsilon}=\mathcal{U}_{\varepsilon}$.

(ii) An alternate proof of Theorem 6 would be to work directly in the upper half-plane $\left(x_{1}, r\right)$, where $r=\left|x^{\prime}\right|$, at the cost of introducing a degenerate elliptic operator. Since this approach is basically two-dimensional, the results of the Appendix could possibly be replaced by easier two-dimensional analysis. 


\section{The approximating problems}

The main purpose of this section is to present the proofs of Proposition 1 and of Lemmas 1 and 2. In particular, we stress the fact that Lemma 2 provides an important upper bound for the Lagrange multiplier $c_{n, \varepsilon}$. This is the first step in order to implement the PDE analysis of the Appendix.

Before we start with the proofs, we wish first to clarify the identification $\Pi_{n} \simeq$ $[-n, n]^{N} \equiv \Omega_{n}$, as well as the notion of unfolding.

Unfolding the torus. We start with the usual definition $\Pi_{n}=\mathbb{R}^{N} /(2 n \mathbb{Z})^{N}$ obtained by the identification $x \sim x^{\prime}$ iff $x-x^{\prime} \in(2 n \mathbb{Z})^{N}$. For a fixed $\alpha=\left(\alpha_{1}, \ldots, \alpha_{N}\right) \in \mathbb{R}^{N}$, the cube $C_{\alpha}:=\prod_{i=1}^{N}\left[-n+\alpha_{i}, n+\alpha_{i}\right.$ [ contains a unique element of each equivalence class ( $C_{\alpha}$ is often termed a fundamental domain); it may therefore be identified with $\Pi_{n}$. Given $\alpha \in \mathbb{R}^{N}$, the unfolding $\tau_{\alpha}$ of $\Pi_{n}$ associated to $\alpha$ is by definition the one-to-one mapping

$$
\tau_{\alpha}: \Pi_{n} \rightarrow \Omega_{n} \equiv\left[-n, n\left[{ }^{N}, \quad p=\left[\left(x_{1}+\alpha_{1}, \ldots, x_{N}+\alpha_{N}\right)\right] \mapsto\left(x_{1}, \ldots, x_{N}\right) .\right.\right.
$$

This corresponds to a translation of the origin in $\mathbb{R}^{N}$, and thus on the torus. For a given function $f$ defined on $\Pi_{n}$, each unfolding $\tau_{\alpha}$ induces a $2 n$-periodic function $f_{\alpha}$ defined on $\Omega_{n}$.

In some computations (in particular dealing with integration by parts for functions which are not necessarily all periodic), we will need to estimate boundary integrals. The following lemma provides a choice of a "good" unfolding of the torus, by averaging.

Lemma 2.1. Let $f \in L^{1}\left(\Pi_{n}\right)$ be given. There exists an unfolding of the torus $\Pi_{n}$ such that

$$
\left|\int_{\partial \Omega_{n}} f_{\alpha}(x) d x\right| \leq \frac{2^{N-1}}{n} \int_{\Omega_{n}}\left|f_{\alpha}(x)\right| d x .
$$

Moreover, for any $0<\sigma<1$ there exists a subset $D_{\sigma}$ of $\Omega_{n}$ of measure larger than $\sigma\left|\Omega_{n}\right|$ such that for any $\alpha \in D_{\sigma}$ we have

$$
\left|\int_{\partial \Omega_{n}} f_{\alpha}(x) d x\right| \leq \frac{C(\sigma)}{n} \int_{\Omega_{n}}\left|f_{\alpha}(x)\right| d x .
$$

Proof. Integrate the left hand side of 44$]$ for $\alpha \in\left[-n, n\left[{ }^{N}\right.\right.$ and use the mean value theorem to get (44). For (45), argue similarly.

[Notice that the trace of $f_{\alpha}$ is well defined for almost every unfolding.] In what follows, we will no longer distinguish $f$ and $f_{\alpha}$; hopefully this will not lead to a confusion.

Proof of Proposition 1. Let $\left(u_{n, \varepsilon}^{k}\right)_{k \in \mathbb{N}}$ be a minimizing sequence for $\left(\mathcal{P}_{n}^{\varepsilon}\right)$. Since $E_{\varepsilon}\left(u_{n, \varepsilon}^{k}\right)$ is uniformly bounded with respect to $k,\left(u_{n, \varepsilon}^{k}\right)_{k \in \mathbb{N}}$ is bounded in $H^{1}\left(\Pi_{n}\right)$ so that up to a subsequence we may assume

$$
u_{n, \varepsilon}^{k} \rightarrow u_{n, \varepsilon} \quad \text { in } H^{1}\left(\Pi_{n}\right) \text { as } k \rightarrow \infty,
$$

for some $u_{n, \varepsilon}$ in $H^{1}\left(\Pi_{n}\right)$. By weak lower semicontinuity and the Rellich compactness theorem, we infer that

$$
E_{\varepsilon}\left(u_{n, \varepsilon}\right) \leq \liminf _{k \rightarrow \infty} E_{\varepsilon}\left(u_{n, \varepsilon}^{k}\right)=I_{n, \varepsilon}
$$


On the other hand, the Rellich compactness theorem also yields

$$
p\left(u_{n, \varepsilon}\right)=\lim _{k \rightarrow \infty} \int_{\Pi_{n}}\left(i u_{n, \varepsilon}^{k}, \partial_{1} u_{n, \varepsilon}^{k}\right)=2 \pi\left|B^{N-1}\right| .
$$

Hence $u_{n, \varepsilon}$ is a minimizer for $\left(\mathcal{P}_{n}^{\varepsilon}\right)$. The Lagrange multiplier rule implies that for some $\lambda_{n, \varepsilon} \in \mathbb{R}$,

$$
d E_{\varepsilon}\left(u_{n, \varepsilon}\right)=\lambda_{n, \varepsilon} \cdot d p\left(u_{n, \varepsilon}\right) .
$$

Define $c_{n, \varepsilon}:=2 \lambda_{n, \varepsilon} /|\log \varepsilon|$. The previous equality is precisely the weak formulation for the equation

$$
i c_{n, \varepsilon}|\log \varepsilon| \frac{\partial u_{n, \varepsilon}}{\partial x_{1}}=\Delta u_{n, \varepsilon}+\frac{1}{\varepsilon^{2}} u_{n, \varepsilon}\left(1-\left|u_{n, \varepsilon}\right|^{2}\right) \quad \text { on } \Pi_{n}
$$

This ends the proof.

Proof of Lemma 1. We will use the test functions $w_{\varepsilon, R}$ constructed in Subsection 1.2 Notice that $E\left(w_{\varepsilon, R}\right)$ and $p\left(w_{\varepsilon, R}\right)$ depend continuously on $R$. It then follows from (15) that

$$
\exists R(\varepsilon)>0: \quad w_{\varepsilon, R(\varepsilon)} \in \Gamma_{n}
$$

for each large enough $n$ and that

$$
R(\varepsilon) \rightarrow 1 \quad \text { as } \varepsilon \rightarrow 0
$$

The conclusion of Lemma 1 then follows from (14).

We now turn to the proof of Lemma 2. As often in elliptic PDE's, Pohozaev's identity (also termed virial identity in the physics literature) leads to useful estimates. In our case, after unfolding it reads (see Lemma A.2

$$
\begin{array}{r}
\frac{N-2}{2} \int_{\Omega_{n}}\left|\nabla u_{n, \varepsilon}\right|^{2}+\frac{N}{4 \varepsilon^{2}} \int_{\Omega_{n}}\left(1-\left|u_{n, \varepsilon}\right|^{2}\right)^{2}-c_{n, \varepsilon} \frac{N-1}{2}|\log \varepsilon| \int_{\Omega_{n}}\left\langle J u_{n, \varepsilon}, \xi_{1}\right\rangle \\
=\int_{\partial \Omega_{n}}\left[n \frac{\left|\nabla u_{n, \varepsilon}\right|^{2}}{2}+\frac{n}{4 \varepsilon^{2}}\left(1-\left|u_{n, \varepsilon}\right|^{2}\right)^{2}-\frac{\partial u_{n, \varepsilon}}{\partial v} \cdot \sum_{i=1}^{N} x_{i} \frac{\partial u_{n, \varepsilon}}{\partial x_{i}}\right]
\end{array}
$$

where $\xi_{1}$ is the 2 -form defined in $\left[13\right.$. Notice that $\xi_{1}$ is not periodic and therefore 46 depends on the choice of unfolding. In order to bound $c_{n, \varepsilon}$, we thus need to provide a lower bound for the quantity

$$
\left|\int_{\Omega_{n}}\left\langle J u_{n, \varepsilon}, \xi_{1}\right\rangle\right|
$$

As we have already noticed in the Introduction (see Remark 2), 47) is related to the momentum $p\left(u_{n, \varepsilon}\right)$ (actually they would even be equal if $u_{n, \varepsilon}$ were constant on $\partial \Omega_{n}$ ). In the situation which is of interest for us, we have the following. 
Lemma 2.2. Let $M_{0}>0$. There exists a constant $K_{2}>0$ (depending only on $\left.M_{0}\right)$ such that for any $n \in \mathbb{N}$ and $u \in H^{1}\left(\Pi_{n}\right)$ satisfying

$$
\left\{\begin{array}{l}
n \geq K_{2}|\log \varepsilon| \varepsilon^{3-N}, \\
E_{\varepsilon}(u) \leq M_{0}|\log \varepsilon|
\end{array}\right.
$$

there exists an unfolding of $\Pi_{n}$ such that

$$
\left|\int_{\Pi_{n}}\left(i u, \partial_{1} u\right)-\int_{\Omega_{n}}\left\langle J u, \xi_{1}\right\rangle\right| \leq r(\varepsilon),
$$

where $r(\varepsilon) \rightarrow 0$ as $\varepsilon \rightarrow 0$, independently of $n$, and

$$
n \int_{\partial \Omega_{n}} e_{\varepsilon}(u) \leq C \int_{\Pi_{n}} e_{\varepsilon}(u) .
$$

Proof. We first claim that there exists $v \in X_{n}$ such that

$$
\|\nabla v\|_{\infty} \leq C / \varepsilon, \quad\|v\|_{\infty} \leq 1, \quad E_{\varepsilon}(v) \leq 2 M_{0}|\log \varepsilon|
$$

and

$$
\left|\int_{\Pi_{n}}\left(i u, \partial_{1} u\right)-\int_{\Pi_{n}}\left(i v, \partial_{1} v\right)\right| \leq r(\varepsilon),
$$

where $r(\varepsilon) \rightarrow 0$ as $\varepsilon \rightarrow 0$, independently of $n$. Indeed, consider first the function $v_{1}$ defined by

$$
v_{1}(x):= \begin{cases}u(x) & \text { if }|u(x)| \leq 1, \\ u(x) /|u(x)| & \text { if not. }\end{cases}
$$

Clearly, $E_{\varepsilon}\left(v_{1}\right) \leq E_{\varepsilon}(u)$ and

$$
\begin{aligned}
\left|\int_{\Pi_{n}}\left(i u, \partial_{1} u\right)-\int_{\Pi_{n}}\left(i v_{1}, \partial_{1} v_{1}\right)\right| & \leq \int_{\Pi_{n}}\left|u-v_{1}\right| \cdot|\nabla u|+\left|\int_{\Pi_{n}}\left(i v_{1}, \partial_{1} u-\partial_{1} v_{1}\right)\right| \\
& =\int_{\Pi_{n}}\left|u-v_{1}\right| \cdot|\nabla u|+\left|\int_{\Pi_{n}}\left(i \partial_{1} v_{1}, u-v_{1}\right)\right| \\
& \leq C\left(\int_{\Pi_{n}}\left|u-v_{1}\right|^{2}\right)^{1 / 2} E_{\varepsilon}(u)^{1 / 2} \\
& \leq C\left(\int_{|u|>1}\left(1-|u|^{2}\right)^{2}\right)^{1 / 2} E_{\varepsilon}(u)^{1 / 2} \\
& \leq C \varepsilon|\log \varepsilon|=: r_{1}(\varepsilon) .
\end{aligned}
$$

Next, consider a function $v_{2}$ defined as a solution of the minimization problem

$$
\min _{w \in H^{1}\left(\Pi_{n}\right)}\left(E_{\varepsilon}(w)+\int_{\Pi_{n}} \frac{\left|w-v_{1}\right|^{2}}{2 \varepsilon}\right) .
$$


Clearly, we also have $E_{\varepsilon}\left(v_{2}\right) \leq E_{\varepsilon}\left(v_{1}\right)$ and

$$
\begin{aligned}
\left|\int_{\Pi_{n}}\left(i v_{1}, \partial_{1} v_{1}\right)-\int_{\Pi_{n}}\left(i v_{2}, \partial_{1} v_{2}\right)\right| & \leq C\left(\int_{\Pi_{n}}\left|v_{1}-v_{2}\right|^{2}\right)^{1 / 2} E_{\varepsilon}\left(v_{1}\right)^{1 / 2} \\
& \leq C \sqrt{\varepsilon} E_{\varepsilon}\left(v_{1}\right)^{1 / 2} E_{\varepsilon}(u)^{1 / 2} \\
& \leq C \sqrt{\varepsilon}|\log \varepsilon|=: r_{2}(\varepsilon) .
\end{aligned}
$$

On the other hand, $v_{2}$ satisfies the equation

$$
\Delta v_{2}+\frac{1}{\varepsilon^{2}} v_{2}\left(1-\left|v_{2}\right|^{2}\right)=\frac{v_{2}-v_{1}}{\varepsilon},
$$

so that $\tilde{v}_{2}(x):=v_{2}(\varepsilon x)$ satisfies

$$
\Delta \tilde{v}_{2}+\tilde{v}_{2}\left(1-\left|\tilde{v}_{2}\right|^{2}\right)=\varepsilon\left(\tilde{v}_{2}-\tilde{v}_{1}\right)
$$

( $\tilde{v}_{1}$ is defined similarly). Since $\left\|\tilde{v}_{1}\right\|_{\infty},\left\|\tilde{v}_{2}\right\|_{\infty} \leq 1$, it follows from standard elliptic estimates that

$$
\left\|\nabla \tilde{v}_{2}\right\|_{\infty} \leq C \quad \text { and so } \quad\left\|\nabla v_{2}\right\|_{\infty} \leq C / \varepsilon .
$$

Combining (48) and (49) we conclude that $v:=v_{2}$ satisfies the conditions of the claim with $r(\varepsilon):=r_{1}(\varepsilon)+r_{2}(\varepsilon)$.

We will now choose a suitable unfolding. Notice first that for any unfolding of $\Pi_{n}$,

$$
\int_{\Pi_{n}}\left(i u, \partial_{1} u\right)-\int_{\Omega_{n}}\left\langle J u, \xi_{1}\right\rangle=n \int_{\partial \Omega_{n}}\left(i u, \partial_{1} u\right) .
$$

By Lemma 2.1. there exists an unfolding such that

$$
\begin{aligned}
\int_{\partial \Omega_{n}}\left(\frac{\left|u-v_{2}\right|^{2}}{\sqrt{\varepsilon}}+\sqrt{\varepsilon}\left(e_{\varepsilon}(u)\right.\right. & \left.\left.+e_{\varepsilon}\left(v_{2}\right)\right)\right) \\
\leq & \frac{2^{N-1}}{n} \int_{\Pi_{n}}\left(\frac{\left|u-v_{2}\right|^{2}}{\sqrt{\varepsilon}}+\sqrt{\varepsilon}\left(e_{\varepsilon}(u)+e_{\varepsilon}\left(v_{2}\right)\right)\right) .
\end{aligned}
$$

Hence, arguing as in 48,

$$
\begin{aligned}
n\left|\int_{\partial \Omega_{n}}\left(\left(i u, \partial_{1} u\right)-\left(i v_{2}, \partial_{1} v_{2}\right)\right)\right| & \leq C \cdot n \int_{\partial \Omega_{n}}\left|u-v_{2}\right| \cdot\left(|\nabla u|+\left|\nabla v_{2}\right|\right) \\
& \leq C \cdot n \int_{\partial \Omega_{n}}\left(\frac{\left|u-v_{2}\right|^{2}}{\sqrt{\varepsilon}}+\sqrt{\varepsilon}\left(e_{\varepsilon}(u)+e_{\varepsilon}\left(v_{2}\right)\right)\right) \\
& \leq C \int_{\Pi_{n}}\left(\frac{\left|u-v_{2}\right|^{2}}{\sqrt{\varepsilon}}+\sqrt{\varepsilon}\left(e_{\varepsilon}(u)+e_{\varepsilon}\left(v_{2}\right)\right)\right) \\
& \leq C \sqrt{\varepsilon}|\log \varepsilon|=r_{2}(\varepsilon) .
\end{aligned}
$$

If $n \geq C_{0} \varepsilon^{3-N}|\log \varepsilon|$, then it follows from 51 that

$$
\int_{\partial \Omega_{n}} e_{\varepsilon}\left(v_{2}\right) \leq \frac{C}{K_{2}} \varepsilon^{3-N} .
$$


It is an easy matter to verify that the last inequality, combined with the estimate $\left|\nabla v_{2}\right|_{\infty} \leq$ $C / \varepsilon$, implies that for $K_{2}$ sufficiently large,

$$
\left|v_{2}(x)\right| \geq 1 / 2 \quad \text { for all } x \in \partial \Omega_{n} .
$$

We may thus write $v_{2}=\rho \exp (i \varphi)$ on $\partial \Omega_{n}$ and from 53 it follows that $\varphi$ is $2 n$-periodic (see step 4 of Theorem 4 in Appendix $\mathrm{C}$ for a detailed proof of this last statement). Hence,

$$
\begin{aligned}
\left|\int_{\partial \Omega_{n}}\left(i v_{2}, \partial v_{2}\right)\right| & =\left|\int_{\partial \Omega_{n}} \rho^{2} \partial_{1} \varphi\right|=\left|\int_{\partial \Omega_{n}}\left(\rho^{2}-1\right) \partial_{1} \varphi\right| \\
& \leq C \varepsilon\left(\int_{\partial \Omega_{n}} \frac{\left(\rho^{2}-1\right)^{2}}{\varepsilon^{2}}\right)^{1 / 2} \cdot\left(\int_{\partial \Omega_{n}}\left|\nabla v_{2}\right|^{2}\right)^{1 / 2} \\
& \leq C \varepsilon \frac{E_{\varepsilon}\left(v_{2}\right)}{n}=\frac{r_{1}(\varepsilon)}{n} .
\end{aligned}
$$

Combining (50), (52) and (54) we finally obtain

$$
\left|\int_{\Pi_{n}}\left(i u, \partial_{1} u\right)-\int_{\Omega_{n}}\left\langle J u, \xi_{1}\right\rangle\right| \leq r_{2}(\varepsilon)+r_{1}(\varepsilon),
$$

which finishes the proof.

We are now in a position to obtain the expected upper bound for the Lagrange multiplier $c_{n, \varepsilon}$.

Proof of Lemma 2. We deduce from (46) that for each unfolding we have

$$
c_{n, \varepsilon}|\log \varepsilon|\left|\int_{\Omega_{n}}\left\langle J u_{n, \varepsilon}, \xi_{1}\right\rangle\right| \leq C\left[n \int_{\partial \Omega_{n}} e_{\varepsilon}\left(u_{n, \varepsilon}\right)+\int_{\Pi_{n}} e_{\varepsilon}\left(u_{n, \varepsilon}\right)\right] .
$$

By Lemma 2.2, there exists an unfolding such that

$$
n \int_{\partial \Omega_{n}} e_{\varepsilon}(u) \leq C \int_{\Pi_{n}} e_{\varepsilon}(u)
$$

and

$$
\left|\int_{\Pi_{n}}\left(i u, \partial_{1} u\right)-\int_{\Omega_{n}}\left\langle J u, \xi_{1}\right\rangle\right| \leq \pi\left|B^{N-1}\right|
$$

provided $\varepsilon$ is chosen sufficiently small and $n \geq n(\varepsilon)$. Therefore, since $u_{n, \varepsilon}$ satisfies the constraint $\int_{\Pi_{n}}\left(i u_{n, \varepsilon}, \partial_{1} u_{n, \varepsilon}\right)=2 \pi\left|B^{N-1}\right|$, we obtain

$$
\left|\int_{\Omega_{n}}\left\langle J u, \xi_{1}\right\rangle\right| \geq \pi\left|B^{N-1}\right|
$$

Combining 55- -57 we deduce

$$
\left|c_{n, \varepsilon}\right| \leq C \frac{E_{\varepsilon}\left(u_{n, \varepsilon}\right)}{|\log \varepsilon|} \leq K_{1},
$$

where we have used Lemma 1 for the last inequality. 


\section{Relation with the isoperimetric problem}

In this section, we specify the geometrical interpretation of both the momentum and the energy, in the asymptotic limit $\varepsilon \rightarrow 0$. Roughly speaking, for $N=3, E_{\varepsilon}\left(u_{n, \varepsilon}\right)$ is proportional to the length of the concentration set, whereas $p\left(u_{n, \varepsilon}\right)$ is proportional to the flux (along $\mathbf{e}_{1}$ ) through the concentration set. As we emphasized in the introduction, the concepts of geometric measure theory are appropriate to express these properties.

We start with the proof of Lemma 5. Recall that in view of Theorem 4, there exist $\ell \in \mathbb{N}, R>0$ and $q$ points $x_{1, \varepsilon}, \ldots, x_{q, \varepsilon}$ with $q \leq l$ such that

$$
\left|u_{n, \varepsilon}(x)\right| \geq 1 / 2 \quad \text { on } \Pi_{n} \backslash \bigcup_{i=1}^{q} B\left(x_{i, \varepsilon}, R\right) .
$$

Without loss of generality, we may assume that the balls $B\left(x_{i, \varepsilon}, 8 R\right)$ are disjoint. For a map $u \in H^{1}\left(\Pi_{n}, \mathbb{C}\right)$, let $\tilde{u}$ be defined by

$$
\tilde{u}(x):= \begin{cases}u(x) & \text { if } x \in \bigcup_{i=1}^{q} B\left(x_{i, \varepsilon}, R\right), \\ \lambda(x) u(x)+(1-\lambda(x)) \bar{u}(x) & \text { if } x \in \bigcup_{i=1}^{q} B\left(x_{i, \varepsilon}, 2 R\right) \backslash B\left(x_{i, \varepsilon}, R\right), \\ \bar{u}(x) & \text { otherwise, }\end{cases}
$$

where

$$
\bar{u}(x):= \begin{cases}u(x) /|u(x)| & \text { if }|u(x)| \geq 1 / 2, \\ 2 u(x) & \text { otherwise, }\end{cases}
$$

and $\lambda(x):=\left(2 R-\left|x-x_{i, \varepsilon}\right|\right) / R$ if $x \in B\left(x_{i, \varepsilon}, 2 R\right) \backslash B\left(x_{i, \varepsilon}, R\right)$. In view of 58 ,

$$
J \tilde{u}_{n, \varepsilon}=0 \quad \text { on } \Pi_{n} \backslash \bigcup_{i=1}^{q} B\left(x_{i, \varepsilon}, 2 R\right)
$$

and

$$
\int_{B\left(x_{i, \varepsilon}, 2 R\right)} J \tilde{u}_{n, \varepsilon}=0
$$

this last inequality follows by integration by parts, by using the fact that $\left|\tilde{u}_{n, \varepsilon}\right|=1$ on $\partial B\left(x_{i, \varepsilon}, 2 R\right)$. These localization properties of $J \tilde{u}_{n, \varepsilon}$ will be useful below. On the other hand, $J \tilde{u}_{n, \varepsilon}$ and $J u_{n, \varepsilon}$ are close in view of the following lemma.

Lemma 3.1. Let $u \in H^{1}\left(\Pi_{n}, \mathbb{C}\right)$ be such that $E_{\varepsilon}(u) \leq M_{0}|\log \varepsilon|$. Then there exists an unfolding of $\Pi_{n}$ such that for every $\varphi \in \mathcal{C}^{\infty}\left(\Omega_{n}, \Lambda^{2} \mathbb{R}^{N}\right)$, we have

$$
\left|\int_{\Omega_{n}}\langle J u-J \tilde{u}, \varphi\rangle\right| \leq\left(\frac{1}{n}\|\varphi\|_{\infty}+\left\|d^{*} \varphi\right\|_{\infty}\right) C \varepsilon|\log \varepsilon|,
$$

and in particular

$$
\|J u-J \tilde{u}\|_{\left[\mathcal{C}^{0,1}\left(\Pi_{n}\right)\right]^{*}} \leq C \varepsilon|\log \varepsilon|,
$$

where $C$ depends only on $N$ and $M_{0}$ but is independent of $n$. 
Proof. According to Lemma 2.1 there exists an unfolding of $\Pi_{n}$ such that

$$
\int_{\partial \Omega_{n}}|u \times d u-\tilde{u} \times d \tilde{u}| \leq \frac{2^{N-1}}{n} \int_{\Omega_{n}}|u \times d u-\tilde{u} \times d \tilde{u}| .
$$

Let $\varphi \in \mathcal{C}^{\infty}\left(\Omega_{n}, \Lambda^{2} \mathbb{R}^{N}\right)$. Integrating by parts on $\Omega_{n}$, we obtain

$\int_{\Omega_{n}}\langle J u-J \tilde{u}, \varphi\rangle=\frac{1}{2} \int_{\partial \Omega_{n}}(u \times d u-\tilde{u} \times d \tilde{u})_{\top} \wedge(* \varphi)_{\top}-\frac{1}{2} \int_{\Omega_{n}}\left\langle u \times d u-\tilde{u} \times d \tilde{u}, d^{*} \varphi\right\rangle$.

Hence, we deduce from 63 that

$$
\left|\int_{\Omega_{n}}\langle J u-J \tilde{u}, \varphi\rangle\right| \leq C\left(\frac{2^{N-1}}{n}\|\varphi\|_{\infty}+\left\|d^{*} \varphi\right\|_{\infty}\right)\|u \times d u-\tilde{u} \times d \tilde{u}\|_{L^{1}\left(\Omega_{n}\right)} .
$$

The proof is completed by using the estimate for $\|u \times d u-\tilde{u} \times d \tilde{u}\|_{L^{1}\left(\Omega_{n}\right)}$ given in the next lemma.

Lemma 3.2. There exists an absolute constant $C>0$ such that

$$
\|u \times d u-\tilde{u} \times d \tilde{u}\|_{L^{1}\left(\Omega_{n}\right)} \leq C \varepsilon E_{\varepsilon}(u) .
$$

Proof. Let $A=\{|u| \geq 1 / 2\}, B=\Omega \backslash A$. A simple computation gives

$$
\begin{aligned}
\|u \times d u-\tilde{u} \times d \tilde{u}\|_{L^{1}(A)} & \leq C \int_{A}\left|\left(1-\frac{1}{|u|^{2}}\right) u \times d u\right| \\
& \leq C \varepsilon\left(\int_{\Omega_{n}} \frac{\left(1-|u|^{2}\right)^{2}}{\varepsilon^{2}}\right)^{1 / 2}\left(\int_{\Omega_{n}}|\nabla u|^{2}\right)^{1 / 2} \\
& \leq C \varepsilon E_{\varepsilon}(u) .
\end{aligned}
$$

On the other hand, we have

$$
\begin{aligned}
\|u \times d u-\tilde{u} \times d \tilde{u}\|_{L^{1}(B)} & \leq C \int_{B}|u \times d u| \leq C|B|^{1 / 2}\left(\int_{\Omega_{n}}|\nabla u|^{2}\right)^{1 / 2} \\
& \leq C \varepsilon\left(\int_{\Omega_{n}} \frac{\left(1-|u|^{2}\right)^{2}}{\varepsilon^{2}}\right)^{1 / 2} E_{\varepsilon}(u)^{1 / 2} \leq C \varepsilon E_{\varepsilon}(u) .
\end{aligned}
$$

Combining 66, with 67) yields 65.

In view of (59), $J \tilde{u}_{n, \varepsilon}$ is localized in balls $B\left(x_{i, \varepsilon}, 2 R\right)$. Concerning the existence of integral boundaries close to $J \tilde{u}_{\varepsilon}$, we will make use of recent works on the geometry of the Jacobians [29, 1]. In particular the $\Gamma$-convergence results contained in the above quoted works lead to the following.

Lemma 3.3. Let $M_{0}>0, R>0$ and $X:=\left\{u \in H^{1}\left(B_{4 R}, \mathbb{C}\right):|u| \geq 1 / 2\right.$ on $\left.B_{4 R} \backslash B_{R}\right\}$. Then, for every $\delta>0$, there exists $\varepsilon_{0}>0$ (depending only on $\delta, R$ and $M_{0}$ ) such that for any $\varepsilon<\varepsilon_{0}$, and for any $u \in X$ such that $E_{\varepsilon}(u) \leq M_{0}|\log \varepsilon|$, there exists an $(N-2)$ dimensional integral boundary $T_{u}=\partial R_{u}$ supported in $B_{R}$ satisfying 
(i) $\left\|J u-\pi T_{u}\right\|_{\left[\mathcal{C}_{\mathrm{c}}^{0,1}\left(B_{4 R}\right)\right]^{*}} \leq \delta$,

(ii) $\mathbf{M}\left(T_{u}\right) \leq \frac{E_{\varepsilon}(u)}{\pi|\log \varepsilon|}+\delta$.

Proof. We argue by contradiction. Assume that there exists some $\delta>0$, a sequence $\varepsilon_{j} \rightarrow 0$, and maps $u_{j} \in X$ satisfying the bound

$$
E_{\varepsilon_{j}}\left(u_{j}\right) \leq M_{0}\left|\log \varepsilon_{j}\right|
$$

and such that for every integral boundary $T$ supported in $B_{R}$ and satisfying (i), statement (ii) does not hold, i.e.

$$
\mathbf{M}(T)>\frac{E_{\varepsilon}(u)}{\pi|\log \varepsilon|}+\delta .
$$

According to the $\Gamma$-convergence results in [29, 1] (see e.g. Theorem 3.1 and Remark 3.2 in [1]), there exists an integral boundary $T^{*}$ supported in $B_{4 R}$ such that

$$
\left\|J u_{j}-\pi T^{*}\right\|_{\left[\mathcal{C}_{\mathrm{C}}^{0,1}\left(B_{4 R}\right)\right]^{*}} \rightarrow 0 \quad \text { as } j \rightarrow \infty
$$

and

$$
\mathbf{M}(T) \leq \liminf _{\varepsilon_{j} \rightarrow 0} \frac{E_{\varepsilon_{j}}\left(u_{j}\right)}{\pi\left|\log \varepsilon_{j}\right|} .
$$

We deduce from (70) that (i) is satisfied for $T=T^{*}$ and $j$ sufficiently large, so that 71 . contradicts 693 [indeed the fact that $T^{*}$ is supported in $B_{R}$, and therefore can be used as a test current in (i), follows from its construction in [1]].

Proof of Lemma 5 completed. We apply Lemma 3.3 to $\tilde{u}_{n, \varepsilon}$ restricted to the balls $B\left(x_{i, \varepsilon}, 4 R\right.$ ), for $i=1, \ldots, q$. This yields integral boundaries $T_{i}$ (depending of course on $\varepsilon$ and $n)$ supported in $B\left(x_{i, \varepsilon}, R\right)$ such that

$$
\left\|J \tilde{u}_{n, \varepsilon}-\pi T_{i}\right\|_{\left[\mathcal{C}_{\mathrm{c}}^{0,1}\left(B\left(x_{i, \varepsilon}, 4 R\right)\right)\right]^{*}} \leq r(\varepsilon)
$$

and

$$
\mathbf{M}\left(T_{i}\right) \leq \frac{E_{\varepsilon}\left(\tilde{u}_{n, \varepsilon} ; B\left(x_{i, \varepsilon}, 4 R\right)\right)}{\pi|\log \varepsilon|}+r(\varepsilon) \leq \frac{E_{\varepsilon}\left(u_{n, \varepsilon} ; B\left(x_{i, \varepsilon}, 4 R\right)\right)}{\pi|\log \varepsilon|}+r(\varepsilon)
$$

[here and in the following, $r(\varepsilon)$ denotes a generic function such that $r(\varepsilon) \rightarrow 0$ as $\varepsilon \rightarrow 0$, independently of $n$, but whose exact value may differ from place to place]. Set $T=$ $\sum_{i=1}^{q} T_{i}$. By $[73$, ,

$$
\mathbf{M}(T) \leq \frac{E_{\varepsilon}\left(u_{n, \varepsilon}\right)}{\pi|\log \varepsilon|}+r(\varepsilon),
$$

so that (iii) is established. Concerning (i), since $J \tilde{u}_{n, \varepsilon}$ is supported in the balls of radius $2 R$, we deduce from (72) that

$$
\begin{aligned}
\left\|J \tilde{u}_{n, \varepsilon}-\pi T\right\|_{\left.\left[\mathcal{C}^{0,1}\left(\Pi_{n}\right)\right)\right]^{*}} & \leq \sum_{i=1}^{q}\left\|J \tilde{u}_{n, \varepsilon}-\pi T_{i}\right\|_{\left[\mathcal{C}^{0,1}\left(B\left(x_{i, \varepsilon}, 2 R\right)\right)\right]^{*}} \\
& \leq C \sum_{i=1}^{q}\left\|J \tilde{u}_{n, \varepsilon}-\pi T_{i}\right\|_{\left[\mathcal{C}_{\mathfrak{c}}^{0,1}\left(B\left(x_{i, \varepsilon}, 4 R\right)\right)\right]^{*}} \leq r(\varepsilon) .
\end{aligned}
$$


Since $\left\|J u_{n, \varepsilon}-J \tilde{u}_{n, \varepsilon}\right\|_{\left.\left[\mathcal{C}^{0,1}\left(\Pi_{n}\right)\right)\right]^{*}} \leq r(\varepsilon)$ in view of Lemma 3.1, we derive (i) from the previous inequality.

Finally, we turn to (ii). For any unfolding we have

$$
\left|\int_{\Omega_{n}}\left\langle J u_{n, \varepsilon}-\pi T, \xi_{1}\right\rangle\right| \leq\left|\int_{\Omega_{n}}\left\langle J \tilde{u}_{n, \varepsilon}-\pi T, \xi_{1}\right\rangle\right|+\left|\int_{\Omega_{n}}\left\langle J \tilde{u}_{n, \varepsilon}-J u_{n, \varepsilon}, \xi_{1}\right\rangle\right| .
$$

Notice that

$$
\begin{aligned}
\left|\int_{\Omega_{n}}\left\langle J \tilde{u}_{n, \varepsilon}-\pi T, \xi_{1}\right\rangle\right| & \leq \sum_{i=1}^{q}\left|\int_{B\left(x_{i, \varepsilon}, 2 R\right)}\left\langle J \tilde{u}_{n, \varepsilon}-\pi T_{i}, \xi_{1}\right\rangle\right| \\
& =\sum_{i=1}^{q}\left|\int_{B\left(x_{i, \varepsilon}, 2 R\right)}\left\langle J \tilde{u}_{n, \varepsilon}-\pi T_{i}, \xi_{1}-\xi_{1}^{i}\right\rangle\right|,
\end{aligned}
$$

where $\xi_{1}^{i}$ denotes the constant form

$$
\xi_{1}^{i}:=\frac{2}{N-1} \sum_{j=1}^{N}\left(x_{i, \varepsilon}\right)_{j} d x_{i} \wedge d x_{j}
$$

and $\left(x_{i, \varepsilon}\right)_{j}$ denotes the $j$-component of the point $x_{i, \varepsilon}$. For the last inequality, we have used 60. By construction,

$$
\left\|\xi_{1}-\xi_{1}^{i}\right\|_{L^{\infty}\left(B\left(x_{i, \varepsilon}, 2 R\right)\right)} \leq \frac{4 R}{N-1}
$$

(whereas $\left\|\xi_{1}\right\|_{L^{\infty}\left(B\left(x_{i, \varepsilon}, 2 R\right)\right)}$ diverges as $\left.n \rightarrow \infty\right)$. Hence, we obtain the estimate

$$
\left|\int_{B\left(x_{i, \varepsilon}, 2 R\right)}\left\langle J \tilde{u}_{n, \varepsilon}-\pi T_{i}, \xi_{1}-\xi_{1}^{i}\right\rangle\right| \leq C\left\|J \tilde{u}_{n, \varepsilon}-\pi T_{i}\right\|_{\left[\mathcal{C}_{\mathrm{c}}^{0,1}(4 R)\right]^{*}} \leq r(\varepsilon) .
$$

We now choose the particular unfolding given by Lemma 3.1 , and similarly we obtain

$$
\left|\int_{\Omega_{n}}\left\langle J u_{n, \varepsilon}-J \tilde{u}_{n, \varepsilon}, \xi_{1}\right\rangle\right| \leq r(\varepsilon),
$$

so that (ii) follows from $75-78$.

Proof of Proposition 3 . First, observe that

$$
\left|\int_{\Omega_{n}}\left\langle\pi T_{n, \varepsilon}, \xi_{1}\right\rangle\right|=\left|\int_{\Omega_{n}}\left\langle\pi R_{n, \varepsilon}, 2 d x_{1}\right\rangle\right| \leq 2 \pi \mathbf{M}\left(R_{n, \varepsilon}\right) .
$$

In view of (ii) and (iii) of Lemma 5 and 79 , we deduce

$$
\frac{\mathbf{M}\left(T_{n, \varepsilon}\right)^{\frac{N-1}{N-2}}}{\mathbf{M}\left(R_{n, \varepsilon}\right)} \leq \frac{2 \pi E_{\varepsilon}\left(u_{n, \varepsilon}\right)^{\frac{N-1}{N-2}}}{(\pi|\log \varepsilon|)^{\frac{N-1}{N-2}} p\left(u_{n, \varepsilon}\right)}+r(\varepsilon),
$$

where $r(\varepsilon) \rightarrow 0$ as $\varepsilon \rightarrow 0$, independently of $n$. The last inequality together with (17) proves (36). 
Moreover, from Lemma 1 and (35), we deduce

$$
\left|\mathbf{M}\left(T_{n, \varepsilon}\right)-\right| S^{N-2}|| \leq r(\varepsilon), \quad\left|\mathbf{M}\left(R_{n, \varepsilon}\right)-\right| B^{N-1}|| \leq r(\varepsilon),
$$

and

$$
\left|\int_{\Pi_{n}}\left\langle R_{n, \varepsilon}, d x_{1}\right\rangle-\right| B^{N-1}|| \leq r(\varepsilon),
$$

where $r(\varepsilon) \rightarrow 0$ as $\varepsilon \rightarrow 0$, independently of $n$. From [81] we infer in particular (see [20, 4.2.17]) that for any sequences $\varepsilon_{j} \rightarrow 0$ and $n_{j} \geq n\left(\varepsilon_{j}\right)$ there exist subsequences (still denoted $\varepsilon_{j}$ and $n_{j}$ ) and translations $\tau_{j}$ in $\Pi_{n_{j}}$ such that

$$
T_{n_{j}, \varepsilon_{j}} \rightarrow T_{\infty} \quad \text { and } \quad R_{n_{j}, \varepsilon_{j}} \rightarrow R_{\infty} \quad \text { in }\left[\mathcal{C}_{\mathrm{c}}^{0,1}\left(\mathbb{R}^{N}\right)\right]^{*}
$$

as $j \rightarrow \infty$, where $T_{\infty}=\partial R_{\infty}$ satisfies

$$
\frac{\mathbf{M}\left(T_{\infty}\right)^{\frac{N-1}{N-2}}}{\mathbf{M}\left(R_{\infty}\right)}=\lambda_{N}
$$

From 83 and 81 we conclude that $T_{\infty}=S^{N-2}$ and $R_{\infty}=B^{N-1}$. Combining 79 with (82) we also obtain

$$
\left|\int_{\mathbb{R}^{N}}\left\langle R_{\infty}, d x_{1}\right\rangle\right|=\mathbf{M}\left(R_{\infty}\right),
$$

i.e. $R_{\infty}$ is contained in a hyperplane orthogonal to $\mathbf{e}_{1}$. The proof is complete.

Proof of Lemma 6. We claim first that $T_{n, \varepsilon}$ is contained in a single ball $B\left(x_{i, \varepsilon}, R\right)$. The other statements are then direct consequences of Theorem 4.

We argue by contradiction. Assume there exist sequences $\varepsilon_{j} \rightarrow 0$ and $n_{j} \geq n\left(\varepsilon_{j}\right)$ for which the claim is false. In particular, for every $R>0$ and every sequence $x_{j} \in \Pi_{n_{j}}$,

$$
\left(\Pi_{n_{j}} \backslash B\left(x_{j}, R\right)\right) \cap S_{n_{j}, \varepsilon_{j}} \neq \varnothing
$$

for $j$ sufficiently large. By Proposition 3, up to some subsequence we have

$$
\tau_{j} T_{n_{j}, \varepsilon_{j}} \rightarrow S^{N-2}
$$

where $\tau_{j}$ is a translation in $\Pi_{n_{j}}$. Let $x_{n_{j}, \varepsilon_{j}}:=\tau_{j}^{-1}(0)$ and $r>1$ be such that

$$
\left|u_{n_{j}, \varepsilon_{j}}\right| \geq 1 / 2 \quad \text { on } B\left(x_{n_{j}, \varepsilon_{j}}, 4 r\right) \backslash B\left(x_{n_{j}, \varepsilon_{j}}, r\right)
$$

(the fact that such an $r$ always exists follows easily by Theorem 2). From (85) with $R=$ $8 r$ we infer that

$$
\left(\Pi_{n_{j}} \backslash B\left(x_{n_{j}, \varepsilon_{j}}, 8 r\right)\right) \cap S_{n_{j}, \varepsilon_{j}} \neq \emptyset
$$

for $j$ sufficiently large. From Theorem 2 we deduce the inequality

$$
\int_{B\left(x_{n_{j}, \varepsilon_{j}}, 4 r\right)} \frac{e_{\varepsilon_{j}}\left(u_{n_{j}, \varepsilon_{j}}\right)}{\pi\left|\log \varepsilon_{j}\right|} \leq \int_{\Pi_{n_{j}}} \frac{e_{\varepsilon_{j}}\left(u_{n_{j}, \varepsilon_{j}}\right)}{\pi\left|\log \varepsilon_{j}\right|}-\frac{\eta}{\pi},
$$


where $\eta>0$ is the constant given by Theorem 2 for $\sigma=1 / 2$. Taking the limit $j \rightarrow \infty$ we obtain, using respectively (86), Lemma 3.3 (ii) with $\delta:=\eta /(2 \pi)$, and (17),

$$
\begin{aligned}
\left|S^{N-2}\right| & \leq \liminf _{j \rightarrow \infty} \int_{B\left(x_{n_{j}, \varepsilon_{j}}, 4 r\right)} \frac{e_{\varepsilon_{j}}\left(u_{n_{j}, \varepsilon_{j}}\right)}{\pi\left|\log \varepsilon_{j}\right|}+\frac{\eta}{2 \pi} \\
& \leq \liminf _{j \rightarrow \infty} \frac{E_{\varepsilon_{j}}\left(u_{n_{j}, \varepsilon_{j}}\right)}{\pi\left|\log \varepsilon_{j}\right|}-\frac{\eta}{\pi}+\frac{\eta}{2 \pi} \leq\left|S^{N-2}\right|-\frac{\eta}{2 \pi} .
\end{aligned}
$$

This is a contradiction.

\section{Proof of Theorem 1 completed}

Recall that in Section 1.5 of the introduction we have already constructed, for $0<\varepsilon<\varepsilon_{0}$ small but fixed, a subsequence of $u_{n, \varepsilon}$ (still denoted here $u_{n, \varepsilon}$ ) such that

$$
\left\{\begin{array}{l}
u_{n, \varepsilon} \rightarrow u_{\varepsilon} \text { strongly in } H_{\mathrm{loc}}^{1}\left(\mathbb{R}^{N}\right), \\
c_{n, \varepsilon} \rightarrow c_{\varepsilon} \text { in } \mathbb{R},
\end{array}\right.
$$

as $n \rightarrow \infty$. Moreover, as $\varepsilon \rightarrow 0$, we have

$$
J u_{\varepsilon} \rightarrow \pi S^{N-2}
$$

and $u_{\varepsilon}$ is a solution on $\mathbb{R}^{N}$ of

$$
i c_{\varepsilon}|\log \varepsilon| \frac{\partial u_{\varepsilon}}{\partial x_{1}}=\Delta u_{\varepsilon}+\frac{1}{\varepsilon^{2}} u_{\varepsilon}\left(1-\left|u_{\varepsilon}\right|^{2}\right) .
$$

In view of (88), $u_{\varepsilon}$ is nontrivial (nonconstant) at least for small $\varepsilon$.

Theorem 11 is stated with $U_{\varepsilon}(x):=u_{\varepsilon}(\varepsilon x)$. We will prove the equivalent statements for $u_{\varepsilon}$; it is then straightforward to come back to $U_{\varepsilon}$. We decompose the remainder of the proof into several steps.

Step 1. We have

$$
\limsup _{n \rightarrow \infty}\left|E_{\varepsilon}\left(u_{\varepsilon}\right)-E_{\varepsilon}\left(u_{n, \varepsilon}\right)\right| \leq C,
$$

where $C$ is independent of $\varepsilon$.

Proof. This is a direct consequence of Theorem 4 and of the strong $H_{\text {loc }}^{1}$ convergence at $\varepsilon$ fixed.

Step 2. We have

$$
\frac{E_{\varepsilon}\left(u_{\varepsilon}\right)}{\pi|\log \varepsilon|}=\left|S^{N-2}\right|+r(\varepsilon),
$$

where $r(\varepsilon) \rightarrow 0$ as $\varepsilon \rightarrow 0$.

Proof. This is a direct consequence of Step 1, Lemma 1 , assertion (iii) of Lemma 5 , and (36).

Step 3. Similarly, we have

$$
p\left(u_{\varepsilon}\right)=p\left(u_{n, \varepsilon}\right)+r(\varepsilon)=2 \pi\left|B^{N-1}\right|+r(\varepsilon)
$$

where $r(\varepsilon) \rightarrow 0$ as $\varepsilon \rightarrow 0$, independently of $n$. 
Proof. Recall that by Lemma 6 .

$$
\left|u_{n, \varepsilon}\right| \geq 1 / 2 \quad \text { on } \Pi_{n} \backslash B(0, R),
$$

so that we may write

$$
u_{n, \varepsilon}=\rho_{n, \varepsilon} \exp \left(i \varphi_{n, \varepsilon}\right) \quad \text { on } \Pi_{n} \backslash B(0, R) .
$$

The definition of $p\left(u_{\varepsilon}\right)$ is then given by (see 38 )

$$
p\left(u_{\varepsilon}\right)=\int_{\mathbb{R}^{N}}\left(i u_{\varepsilon}, \partial_{1} u_{\varepsilon}\right) \chi+\int_{\mathbb{R}^{N}}(1-\chi)\left(\rho_{\varepsilon}^{2}-1\right) \partial_{1} \varphi_{\varepsilon}+\int_{\mathbb{R}^{N}} \varphi_{\varepsilon} \partial_{1}(1-\chi),
$$

where $\chi$ is an arbitrary smooth function with compact support such that $\chi \equiv 1$ on $B_{R}(0)$ and $0 \leq \chi \leq 1$. On the other hand, we have, for $n$ sufficiently large,

$$
p\left(u_{n, \varepsilon}\right)=\int_{\mathbb{R}^{N}}\left(i u_{n, \varepsilon}, \partial_{1} u_{n, \varepsilon}\right) \chi+\int_{\Omega_{n}}(1-\chi)\left(\rho_{n, \varepsilon}^{2}-1\right) \partial_{1} \varphi_{n, \varepsilon}+\int_{\mathbb{R}^{N}} \varphi_{n, \varepsilon} \partial_{1}(1-\chi)
$$

By strong $H_{\text {loc }}^{1}$ convergence, the first and third terms on the right hand side of 91 converge to the corresponding terms in 90 . For the second term, we have

$$
\begin{aligned}
\left|\int_{\Omega_{n}}(1-\chi)\left(\rho_{n, \varepsilon}^{2}-1\right) \partial_{1} \varphi_{n, \varepsilon}\right| & \leq\left(\int_{\Omega_{n}}\left(\rho_{n, \varepsilon}^{2}-1\right)^{2}\right)^{1 / 2}\left(\int_{\Omega_{n} \backslash B(0, R)}\left|\nabla \varphi_{n, \varepsilon}\right|^{2}\right)^{1 / 2} \\
& \leq C \varepsilon E_{\varepsilon}\left(u_{n, \varepsilon}\right) .
\end{aligned}
$$

A similar estimate holds for the second term in 90 , so that the proof is complete.

Step 4. We have

$$
c(\varepsilon) \rightarrow N-2 \quad \text { as } \varepsilon \rightarrow 0 .
$$

Proof. The proof relies (as in Lemma 2) on Pohozaev's identity; however, we are now in a position to use Theorem 4 and Lemma 6 , which provide a better decay of the energy at infinity. Set $B:=B(0, R)$. By Lemma 2.1, there exists an unfolding of the torus such that $\partial \Omega_{n} \cap B=\emptyset$ and

$$
\left|n \int_{\partial \Omega_{n}} e_{\varepsilon}\left(u_{n, \varepsilon}\right)\right| \leq \int_{\Omega_{n}} e_{\varepsilon}\left(u_{n, \varepsilon}\right) \cdot 1_{\Omega_{n} \backslash B} \leq C,
$$

the last inequality being a consequence of Theorem 4 . On the other hand, by Corollary A.1 of the Appendix, we know that

$$
\int_{\Pi_{n}} \frac{\left(1-\left|u_{n, \varepsilon}\right|^{2}\right)^{2}}{\varepsilon^{2}}=o(|\log \varepsilon|) \quad \text { as } \varepsilon \rightarrow 0 .
$$

Finally, using Lemma 2.2 we may choose our unfolding so that it satisfies the additional condition

$$
\left|\int_{\Pi_{n}}\left(\left(i u_{n, \varepsilon}, \partial_{1} u_{n, \varepsilon}\right)-\left\langle J u_{n, \varepsilon}, \xi_{1}\right\rangle\right)\right| \leq r(\varepsilon) .
$$


Hence, by Step 2,

$$
\int_{\Pi_{n}}\left\langle J u_{n, \varepsilon}, \xi_{1}\right\rangle=p\left(u_{n, \varepsilon}\right)+r(\varepsilon)=2 \pi\left|B^{N-1}\right|+r(\varepsilon) .
$$

Going back to 46 , we have by 92 , for fixed $\varepsilon$,

$$
\left.\left|\frac{N-2}{2} \int_{\Omega_{n}}\right| \nabla u_{n, \varepsilon}\right|^{2}+\frac{N}{4 \varepsilon^{2}} \int_{\Omega_{n}}\left(1-\left|u_{n, \varepsilon}\right|^{2}\right)^{2}-c_{n, \varepsilon} \frac{N-1}{2}|\log \varepsilon| \int_{\Omega_{n}}\left\langle J u_{n, \varepsilon}, \xi_{1}\right\rangle \mid \leq C .
$$

Dividing by $|\log \varepsilon|$ and using 93 and 94 we are led to

$$
\frac{N-1}{2} p\left(u_{n, \varepsilon}\right) c_{n, \varepsilon}=(N-2) \frac{E_{\varepsilon}\left(u_{n, \varepsilon}\right)}{|\log \varepsilon|}+r(\varepsilon) \text {. }
$$

The conclusion follows from Steps 2 and 3.

From now on, we will not consider $u_{n, \varepsilon}$ any more in this section, and derive asymptotic properties of $u_{\varepsilon}$ as $\varepsilon$ goes to zero.

Step 5. Up to a subsequence, there exists some map $U_{*} \in W_{\mathrm{loc}}^{1, p}\left(\mathbb{R}^{N}, S^{1}\right)(1 \leq p<$ $N /(N-1))$ such that

$$
u_{\varepsilon} \rightarrow U_{*} \quad \text { weakly in } W_{\text {loc }}^{1, p}\left(\mathbb{R}^{N}\right) \text { as } \varepsilon \rightarrow 0,
$$

where $U_{*}$ is defined (up to a constant phase) in the statement of Theorem 1 .

Proof. By Theorem 4 (i), $u_{\varepsilon}$ is bounded in $W_{\text {loc }}^{1, p}\left(\mathbb{R}^{N}\right)$. Therefore, up to a subsequence, there exists some map $u_{*} \in W_{\text {loc }}^{1, p}\left(\mathbb{R}^{N}\right)$ such that $u_{\varepsilon} \rightarrow u_{*}$ weakly in $W_{\text {loc }}^{1, p}\left(\mathbb{R}^{N}\right)$ and almost everywhere. Moreover,

$$
\int_{\mathbb{R}^{N} \backslash B_{R}(0)}\left|\nabla u_{*}\right|^{2} \leq C
$$

since the same inequality holds for $u_{\varepsilon}$. We next show that $u_{*}=U_{*}$. Since $u_{\varepsilon}$ satisfies equation (89), taking the exterior product of $(89)$ with $u_{\varepsilon}$ and $i u_{\varepsilon}$ respectively we are led to

$$
\left\{\begin{array}{l}
d^{*}\left(u_{\varepsilon} \times d u_{\varepsilon}\right)=i c_{\varepsilon}|\log \varepsilon| \frac{\partial}{\partial x_{1}}\left(\left|u_{\varepsilon}\right|^{2}-1\right), \\
d\left(u_{\varepsilon} \times d u_{\varepsilon}\right)=2 J u_{\varepsilon}
\end{array}\right.
$$

Passing to the limit $\varepsilon \rightarrow 0$ [notice that $c_{\varepsilon}|\log \varepsilon|\left(\left|u_{\varepsilon}\right|^{2}-1\right) \rightarrow 0$ in $L^{2}\left(\mathbb{R}^{N}\right)$ so that the right hand side of the first equation above converges to zero in $H^{-1}\left(\mathbb{R}^{N}\right)$ ], we obtain

$$
\left\{\begin{array}{l}
d^{*}\left(u_{*} \times d u_{*}\right)=0 \\
d\left(u_{*} \times d u_{*}\right)=2 \pi S^{N-2}
\end{array}\right.
$$

This elliptic system together with 55 determines $u_{*}$ uniquely (up to a constant phase). Indeed, from the first equation and classical Hodge-de Rham theory (see e.g. the Appendix of [10]) there exists a 2-form $\psi$ such that

$$
u_{*} \times d u_{*}=d^{*} \psi, \quad d \psi=0, \quad \nabla \psi \in L^{2}\left(\mathbb{R}^{N} \backslash B_{R}(0)\right) .
$$


Inserting this in the second equation satisfied by $u_{*}$ we obtain

$$
\Delta \psi=2 \pi S^{N-2}
$$

so that $\psi=\psi_{*}\left(\psi_{*}\right.$ is defined before Theorem 1$)$, and the conclusion follows.

Step 6. Let $K \subset \mathbb{R}^{N} \backslash S^{N-2}$ be compact and simply connected. For $\varepsilon$ sufficiently small, we have

$$
\left|u_{\varepsilon}(x)\right| \geq 1 / 2 \quad \text { on } K \text {. }
$$

Proof. We apply Theorem 3 with the sequence $\left(u_{\varepsilon}\right)_{\varepsilon>0}$. Indeed, we have $\Sigma_{\mu}=S^{N-2}$ [this can be established arguing as in the proof of Lemma 4]. The claim then follows directly for (27).

We may now write

$$
u_{\varepsilon}(x):=\rho_{\varepsilon}(x) \exp \left(i \varphi_{\varepsilon}(x)\right) \quad \text { on } K .
$$

For convenience we skip the subscripts $\varepsilon$ in what follows. It remains to prove the stronger convergence in the compact set $K$. In contrast with the case $c=0$, where $\left(1-|u|^{2}\right) / \varepsilon^{2}$ remains bounded as $\varepsilon$ goes to zero (see [8]), this is not the case here (it diverges like $|\log \varepsilon|)$. We rely instead on a cancellation effect.

Step 7. We have

(i) $\|\nabla \varphi\|_{\mathcal{C}^{k}(K)} \leq C_{k} \quad \forall k \geq 0$,

(ii) $\left\|\frac{2(1-\rho)}{\varepsilon^{2}}+c_{\varepsilon}|\log \varepsilon| \frac{\partial \varphi}{\partial x_{1}}\right\|_{\mathcal{C}^{k}(K)} \leq C_{k} \quad \forall k \geq 0$.

In particular

$$
\|\nabla \rho\|_{\mathcal{C}^{k}(K)} \leq C_{k} \varepsilon^{2}|\log \varepsilon| \quad \forall k \geq 0
$$

and the convergence claim in Theorem 1 follows from (97) and 99].

Proof. The first important point is to obtain uniform $\mathcal{C}^{0, \alpha}$ bounds, namely

$$
\|u\|_{\mathcal{C}^{0, \alpha}(K)} \leq C
$$

for some $\alpha>0$. This is achieved as in [10, Theorem IV.1], by obtaining first a monotonicity property

$$
\tilde{E}_{\varepsilon}\left(\delta r, x_{0}\right) \leq \frac{1}{2} \tilde{E}_{\varepsilon}\left(r, x_{0}\right) \quad \text { for all } 0<r<r_{0},
$$

for every $x_{0} \in K$ and for some $\delta>0$, and then using the Morrey embedding theorem. We skip the details [see however Step 1 of the proof of Theorem 3 for a very similar proof to obtain [B-9] ]. The analysis of the further regularity properties of $u$ is long and technical in the case of the general equation (19). For equation (89), we make use of the following trick which gives rather directly some first rough (in the sense "nonuniform") estimates for all the derivatives (see also [19]). The remaining analysis is then substantially simplified.

Let $v:=\exp \left(-i c_{\varepsilon}|\log \varepsilon| x_{1}\right) u$. Then $v$ satisfies the equation

$$
\Delta v+\frac{1}{\varepsilon^{2}} v\left(h_{\varepsilon}-|v|^{2}\right)=0
$$


where $h_{\varepsilon}:=1+c_{\varepsilon}^{2} \varepsilon^{2}|\log \varepsilon|^{2}$. Set $w(x):=\left(h_{\varepsilon}\right)^{-1 / 2} v(x)$ and $\tilde{w}(x):=w(x /|\log \varepsilon|)$. We have

$$
\Delta \tilde{w}+\frac{1}{\tilde{\varepsilon}} \tilde{w}\left(1-|\tilde{w}|^{2}\right)=0,
$$

where $\tilde{\varepsilon}^{2}:=\varepsilon^{2}|\log \varepsilon|^{2} h_{\varepsilon}^{-1}$. By 100 and the construction of $\tilde{w}$,

$$
\|\tilde{w}\|_{\mathcal{C}_{\text {loc }}^{0, \alpha}} \leq C .
$$

Using the regularity theory for the Ginzburg-Landau equation, we thus infer (see [10, Theorem IV.1]) that

$$
\|\tilde{w}\|_{\mathcal{C}_{\mathrm{loc}}^{k}} \leq C_{k}, \quad\left\|\frac{1-|\tilde{w}|^{2}}{\tilde{\varepsilon}^{2}}\right\|_{\mathcal{C}_{\mathrm{loc}}^{k}} \leq C_{k}
$$

for all $k \geq 0$. Coming back to $u$, this yields

$$
\|u\|_{\mathcal{C}_{\mathrm{loc}}^{k}} \leq C_{k}|\log \varepsilon|^{k}, \quad\left\|\frac{1-|u|^{2}}{\varepsilon^{2}}\right\|_{\mathcal{C}_{\mathrm{loc}}^{k}} \leq C_{k}|\log \varepsilon|^{2+k} .
$$

Starting with these rough estimates we are now going to prove (97) and (98) using a bootstrap argument. Define

$$
B_{\varepsilon}:=2(1-\rho)+c_{\varepsilon}|\log \varepsilon| \varepsilon^{2} \frac{\partial \varphi}{\partial x_{1}}, \quad A_{\varepsilon}:=\varepsilon^{-2} B_{\varepsilon} .
$$

The equations needed for the bootstrap are

$$
\begin{gathered}
\operatorname{div}\left(\rho^{2} \nabla \varphi\right)=c_{\varepsilon}|\log \varepsilon| \frac{\partial}{\partial x_{1}}\left(\rho^{2}-1\right), \\
-\Delta \rho=A_{\varepsilon}+c_{\varepsilon}|\log \varepsilon|(\rho-1) \frac{\partial \varphi}{\partial x_{1}}-\frac{2(1-\rho)^{2}}{\varepsilon^{2}}(\rho+2), \\
-\Delta B_{\varepsilon}+\frac{\rho(1+\rho)}{\varepsilon^{2}} B_{\varepsilon}=2 \rho|\nabla \varphi|^{2}+\rho(\rho-1)|\log \varepsilon| c_{\varepsilon} \frac{\partial \varphi}{\partial x_{1}}-\left(1-\rho^{2}\right) \varepsilon^{2} \Delta \frac{\partial \varphi}{\partial x_{1}} .
\end{gathered}
$$

Since $\rho$ is bounded in $\mathcal{C}^{0, \alpha}$ by 100 , we infer from Schauder regularity theory, 101 and (102) that

$$
\|\nabla \varphi\|_{\mathcal{C}_{\text {loc }}^{0, \alpha}} \leq C .
$$

Using (101) and 105, we deduce that the right hand side of (104) is bounded in $L_{\text {loc }}^{\infty}$. Hence, by (105) and standard arguments,

$$
\left\|B_{\varepsilon}\right\|_{\mathcal{C}_{\text {loc }}^{0}} \leq C \varepsilon^{2} \quad \text { and thus } \quad\left\|A_{\varepsilon}\right\|_{\mathcal{C}_{\text {loc }}^{0}} \leq C .
$$

Using (101) and (106), we deduce from (103) and then from (102) that

$$
\|\nabla \rho\|_{\mathcal{C}_{\text {loc }}^{0, \alpha}} \leq C, \quad\|\nabla \varphi\|_{C_{\text {loc }}^{1, \alpha}} \leq C .
$$

We are now in a position to differentiate (104) once. This leads us, using (101), 107], to the estimate

$$
\left\|B_{\varepsilon}\right\|_{\mathcal{C}_{\text {loc }}^{1}} \leq C \varepsilon^{2}, \quad \text { i.e. }\left\|A_{\varepsilon}\right\|_{\mathcal{C}_{\text {loc }}^{1}} \leq C .
$$

We have thus proved that (i) and (ii) hold for $k=1$. The estimates for the next derivatives are obtained following exactly the same steps. This finishes the proof of Theorem 1 


\section{Proof of Theorem 5}

The main ingredient in the proof of Proposition 4 is the following inequality:

Lemma 5.1. There exists a constant $C>0$ such that

$$
\int_{\Omega_{n} \backslash B(R)} e_{\varepsilon}\left(u_{n, \varepsilon}\right) \leq C R \int_{\partial B(R)} e_{\varepsilon}\left(u_{n, \varepsilon}\right)
$$

for $R \geq 2, n \geq n(\varepsilon)$, and $\varepsilon$ sufficiently small.

Proof. We multiply the equation

$$
\operatorname{div}\left(\rho^{2} \nabla \varphi\right)=-\frac{c_{\varepsilon}}{2}|\log \varepsilon| \frac{\partial}{\partial x_{1}}\left(\rho^{2}-1\right)
$$

by $\varphi-\bar{\varphi}$, where

$$
\bar{\varphi}:=\frac{1}{|\partial B(R)|} \int_{\partial B(R)} \varphi
$$

denotes the mean value of the phase on $\partial B(0, R)$. Integrating by parts on $\Omega_{n} \backslash B(R)$, we obtain

$$
\begin{aligned}
\int_{\Omega_{n} \backslash B(R)} \rho^{2}|\nabla \varphi|^{2}= & \int_{\partial B(R)} \rho^{2} \frac{\partial \varphi}{\partial v}(\varphi-\bar{\varphi})+\frac{c_{\varepsilon}}{2}|\log \varepsilon| \int_{\Omega_{n} \backslash B(R)} \frac{\partial\left(\rho^{2}-1\right)}{\partial x_{1}}(\varphi-\bar{\varphi}) \\
= & \int_{\partial B(R)} \rho^{2} \frac{\partial \varphi}{\partial v}(\varphi-\bar{\varphi})-\frac{c_{\varepsilon}}{2}|\log \varepsilon| \int_{\partial B(R)}\left(\rho^{2}-1\right)(\varphi-\bar{\varphi}) n_{1} \\
& +\frac{c_{\varepsilon}}{2}|\log \varepsilon| \int_{\Omega_{n} \backslash B(R)}\left(1-\rho^{2}\right) \frac{\partial \varphi}{\partial x_{1}} .
\end{aligned}
$$

We estimate each of the three terms on the right hand side separately. For the first term, we invoke the Poincaré-Wirtinger inequality to assert that

$$
\begin{aligned}
\left|\int_{\partial B(R)} \rho^{2} \frac{\partial \varphi}{\partial v}(\varphi-\bar{\varphi})\right| & \leq C\left(\int_{\partial B(R)}|\nabla \varphi|^{2}\right)^{1 / 2}\left(\int_{\partial B(R)}(\varphi-\bar{\varphi})^{2}\right)^{1 / 2} \\
& \leq C R\left(\int_{\partial B(R)}|\nabla \varphi|^{2}\right) \leq C R \int_{\partial B(R)} e_{\varepsilon}\left(u_{n, \varepsilon}\right) .
\end{aligned}
$$

Similarly, we obtain

$$
\begin{aligned}
\frac{c_{\varepsilon}}{2}|\log \varepsilon|\left|\int_{\partial B(R)}\left(\rho^{2}-1\right)(\varphi-\bar{\varphi}) n_{1}\right| & \leq C \varepsilon|\log \varepsilon| R\left(\int_{\partial B(R)} \frac{\left(1-\rho^{2}\right)^{2}}{\varepsilon^{2}} \int_{\partial B(R)}|\nabla \varphi|^{2}\right)^{1 / 2} \\
& \leq C \varepsilon|\log \varepsilon| R \int_{\partial B(R)} e_{\varepsilon}\left(u_{n, \varepsilon}\right)
\end{aligned}
$$

and

$$
\frac{c_{\varepsilon}}{2}|\log \varepsilon|\left|\int_{\Omega_{n} \backslash B(R)}\left(1-\rho^{2}\right) \frac{\partial \varphi}{\partial x_{1}}\right| \leq C \varepsilon|\log \varepsilon| \int_{\Omega_{n} \backslash B(R)} e_{\varepsilon}\left(u_{n, \varepsilon}\right) .
$$


Combining (109)-111) we are led to

$$
\int_{\Omega_{n} \backslash B(R)}|\nabla \varphi|^{2} \leq C R \int_{\partial B(R)} e_{\varepsilon}\left(u_{n, \varepsilon}\right)+C \varepsilon|\log \varepsilon| \int_{\Omega_{n} \backslash B(R)} e_{\varepsilon}\left(u_{n, \varepsilon}\right) .
$$

We now turn to the equation for $\rho$,

$$
-\Delta \rho+\rho|\nabla \varphi|^{2}+c_{\varepsilon}|\log \varepsilon| \rho \frac{\partial \varphi}{\partial x_{1}}=\rho \frac{1-\rho^{2}}{\varepsilon^{2}} .
$$

Multiplying by $\rho^{2}-1$ and integrating by parts on $\Omega_{n} \backslash B(R)$ gives

$$
\begin{aligned}
\int_{\Omega_{n} \backslash B(R)}\left(2 \rho|\nabla \rho|^{2}+\rho \frac{\left(1-\rho^{2}\right)^{2}}{\varepsilon^{2}}\right) & =\int_{\partial B(R)} \frac{\partial \varphi}{\partial \nu}\left(1-\rho^{2}\right) \\
& +c_{\varepsilon}|\log \varepsilon| \int_{\Omega_{n} \backslash B(R)} \rho\left(1-\rho^{2}\right) \frac{\partial \varphi}{\partial x_{1}}+\int_{\Omega_{n} \backslash B(R)} \rho\left(1-\rho^{2}\right)|\nabla \varphi|^{2} .
\end{aligned}
$$

We have

$$
\left|\int_{\partial B(R)} \frac{\partial \varphi}{\partial v}\left(1-\rho^{2}\right)\right| \leq C \varepsilon \int_{\partial B(R)} e_{\varepsilon}\left(u_{n, \varepsilon}\right),
$$

and

$$
c_{\varepsilon}|\log \varepsilon| \int_{\Omega_{n} \backslash B(R)} \rho\left(1-\rho^{2}\right) \frac{\partial \varphi}{\partial x_{1}}|\leq C \varepsilon| \log \varepsilon \mid \int_{\Omega_{n} \backslash B(R)} e_{\varepsilon}\left(u_{n, \varepsilon}\right) .
$$

For the third term, we invoke the fact (see Theorem 1 that $|\nabla \varphi| \leq C$ in $\Omega_{n} \backslash B(R)$ so that

$$
\left.\left|\int_{\Omega_{n} \backslash B(R)} \rho\left(1-\rho^{2}\right)\right| \nabla \varphi\right|^{2}\left|\leq C \int_{\Omega_{n} \backslash B(R)} \rho\left(1-\rho^{2}\right)\right| \nabla \varphi \mid \leq C \varepsilon \int_{\Omega_{n} \backslash B(R)} e_{\varepsilon}\left(u_{n, \varepsilon}\right) .
$$

Combining (113)-(116), we are led to

$$
\int_{\Omega_{n} \backslash B(R)}\left(|\nabla \rho|^{2}+\frac{\left(1-\rho^{2}\right)^{2}}{\varepsilon^{2}}\right) \leq C \varepsilon \int_{\partial B(R)} e_{\varepsilon}\left(u_{n, \varepsilon}\right)+C \varepsilon|\log \varepsilon| \int_{\Omega_{n} \backslash B(R)} e_{\varepsilon}\left(u_{n, \varepsilon}\right) .
$$

Finally, from 112 and (117) we derive the conclusion.

Proof of Proposition 4 Set, for $R>2$,

$$
f_{n}(R):=\int_{\Omega_{n} \backslash B(R)} e_{\varepsilon}\left(u_{n, \varepsilon}\right) .
$$

We infer from Lemma 5.1 that the $f_{n}$ satisfy the differential inequality

$$
f_{n}(s) \leq-C s f_{n}^{\prime}(s) \quad \text { for all } s>2 .
$$

Integrating between 2 and $R$ yields

$$
f_{n}(R) \leq f_{n}(2)\left(\frac{2}{R}\right)^{\lambda}
$$

where $\lambda:=1 / C$. This proves 441. The other statements (42) and 443) follow directly from this decay. 
Remark 5.1. In the previous computations, we have not tried to optimize the constants. Using the best constant in the Poincaré-Wirtinger inequality for 109, we may prove that 41) is valid with any $\lambda<\sqrt{N-1}$ provided $\varepsilon$ is sufficiently small (depending on $\lambda$ ).

Proof of Theorem 5. Equality (39) has already been established in Proposition 4 . For (40), we argue by contradiction and assume it is false. Then there exists $v \in W$ such that

and

$$
E_{\varepsilon}(v)<E_{\varepsilon}\left(u_{\varepsilon}\right)=\lim _{n \rightarrow \infty} E_{\varepsilon}\left(u_{n, \varepsilon}\right)
$$

$$
p(v)=2 \pi\left|B^{N-1}\right| .
$$

If $v$ were constant outside some large ball $B(R)$, then its restriction to $\Omega_{n}$, for $n \geq R$, would be well defined on $\Pi_{n}$ and therefore, in view of $(119)$, a test function for $\left(\mathcal{P}_{n}^{\varepsilon}\right)$. This contradicts (118) for $n$ sufficiently large.

In the general situation, we will construct from $v$ a function $\tilde{v}$, constant outside some large ball $B(R)$, and satisfying

and

$$
p(\tilde{v})=2 \pi\left|B^{N-1}\right|
$$

$$
E_{\varepsilon}(\tilde{v})<E_{\varepsilon}\left(u_{\varepsilon}\right)
$$

so that a contradiction holds similarly.

Construction of $\tilde{v}$. Since $v \in W$, we may write

$$
v=\eta \exp (i \psi) \quad \text { on } \mathbb{R}^{N} \backslash B(R),
$$

provided $R$ is sufficiently large. We begin by the construction of a function $\check{v}_{R}$, constant outside $B(3 R)$, but which will not yet satisfy 120 . For that purpose, consider the functions $\check{\eta}_{R}$ and $\breve{\psi}_{R}$ defined on $\mathbb{R}^{N} \backslash B(R)$ by

$$
\begin{array}{lrl}
\check{\eta}_{R}(x):=\sigma(x) \eta(x)+(1-\sigma(x)) & \text { with } \sigma(x):=\frac{2 R-|x|}{R}, \\
\check{\psi}_{R}(x):=\tau(x) \psi(x)+(1-\tau(x))\left(\frac{1}{|\partial B(R)|} \int_{\partial B(R)} \psi\right) \text { with } \tau(x):=\frac{3 R-|x|}{R} .
\end{array}
$$

Set

$$
\check{v}_{R}(x):= \begin{cases}v(x) & \text { if }|x| \leq R, \\ \check{\eta}_{R}(x) \exp (i \psi(x)) & \text { if } R \leq|x| \leq 2 R, \\ \exp \left(i \breve{\psi}_{R}\right) & \text { if } 2 R \leq|x| \leq 3 R \\ \exp \left(i \frac{1}{|\partial B(R)|} \int_{\partial B(R)} \psi\right) & \text { otherwise. }\end{cases}
$$

Some computation shows that for some constant $C>0$ independent of $R$,

$$
\left|E_{\varepsilon}\left(\check{v}_{R}\right)-E_{\varepsilon}(v)\right|+\left|p\left(\check{v}_{R}\right)-p(v)\right| \leq C\left[\int_{\partial B(R)} e_{\varepsilon}(v)+\int_{\Omega_{n} \backslash B(R)} e_{\varepsilon}(v)\right] .
$$

We may next take a sequence $\left(R_{m}\right)_{m \in \mathbb{N}}$ such that $R_{m} \rightarrow \infty$ and

$$
\int_{\partial B\left(R_{m}\right)} e_{\varepsilon}(v) \rightarrow 0 \quad \text { as } m \rightarrow \infty
$$


so that

$$
p\left(\check{v}_{R_{m}}\right)=2 \pi\left|B^{N-1}\right|+o(1) \quad \text { as } m \rightarrow \infty
$$

and

$$
E_{\varepsilon}\left(\check{v}_{R_{m}}\right)=E_{\varepsilon}(v)+o(1) \quad \text { as } m \rightarrow \infty .
$$

We finally complete the construction of $\tilde{v}$ by setting

$$
\tilde{v}_{R_{m}}(x):=\check{v}_{R_{m}}\left(\alpha_{m} x\right)
$$

where $\alpha_{m}>0$ is uniquely defined by the relation $p\left(\tilde{v}_{R_{m}}\right)=2 \pi\left|B^{N-1}\right|$. It follows from 122 that $\alpha_{m}=1+o(1)$ as $m \rightarrow \infty$. Hence, if we choose $\tilde{v}:=\tilde{v}_{R_{m}}$, we verify that $\tilde{v}$ satisfies the required conditions for $m$ sufficiently large.

\section{Appendices}

The purpose of these appendices is to develop the asymptotic analysis of the equation

$$
i|\log \varepsilon| \mathbf{c}(x) \cdot \nabla w=\Delta w+\frac{1}{\varepsilon^{2}} w\left(1-|w|^{2}\right)-|\log \varepsilon|^{2} d(x) w \quad \text { on } \Omega,
$$

where $\Omega \subseteq \mathbb{R}^{N}$ is a piecewise $\mathcal{C}^{1}$ simply connected domain, c : $\bar{\Omega} \rightarrow \mathbb{R}^{N}$ is a bounded Lipschitz vector field and $d: \bar{\Omega} \rightarrow \mathbb{R}$ is Lipschitz, nonnegative and bounded. The main results of this analysis have been stated in Lemmas 3 and 4, Proposition 2 and Theorems 2, 3 and 4 We will provide proofs here. Notice that (124) can be rewritten as

$$
i|\log \varepsilon| \mathbf{c}(x) \cdot \nabla w=\Delta w+\frac{1}{\varepsilon^{2}} w\left(a_{\varepsilon}(x)-|w|^{2}\right),
$$

where

$$
a_{\varepsilon}(x):=1-d(x) \varepsilon^{2}|\log \varepsilon|^{2} .
$$

When $\operatorname{div} \mathbf{c}=0$ it is also equivalent to

$$
\left(\nabla-i|\log \varepsilon| \frac{\mathbf{c}}{2}\right)^{2} w+\frac{1}{\varepsilon^{2}} w\left(b_{\varepsilon}(x)-|w|^{2}\right)=0,
$$

where

$$
b_{\varepsilon}(x):=a_{\varepsilon}(x)+\varepsilon^{2}|\log \varepsilon|^{2} \frac{c^{2}(x)}{4} .
$$

In what follows, we assume throughout that

$$
\operatorname{div} \mathbf{c}=0 .
$$

\section{Appendix A: the PDE analysis}

In this first appendix we establish some basic estimates, in particular we give the proof of Lemma 3 
Proof of Lemma 3 Let $w$ satisfy (124) and $\rho(x):=|w(x)|$. Then we have

$$
\begin{aligned}
\Delta \rho^{2} & =(2 w, \Delta w)+2|\nabla w|^{2} \\
& =-\frac{2}{\varepsilon^{2}} \rho^{2}\left(a_{\varepsilon}(x)-\rho^{2}\right)+|\log \varepsilon|(2 i w, \mathbf{c} \cdot \nabla w)+2|\nabla w|^{2} \\
& \geq-\frac{2}{\varepsilon^{2}} \rho^{2}\left(a_{\varepsilon}(x)-\rho^{2}\right)+\left(\sqrt{2}|\nabla w|-\frac{1}{\sqrt{2}} c_{\infty}|\log \varepsilon||w|\right)^{2}-\frac{1}{2} c_{\infty}^{2}|\log \varepsilon|^{2} \rho^{2} \\
& \geq-\frac{2}{\varepsilon^{2}} \rho^{2}\left(b_{\varepsilon}^{\infty}-\rho^{2}\right),
\end{aligned}
$$

where $b_{\varepsilon}^{\infty}:=\left\|b_{\varepsilon}\right\|_{L^{\infty}(K)}$. Hence the function $W(x):=\rho^{2}(x)-b_{\varepsilon}^{\infty}$ satisfies the inequality

$$
\Delta W \geq \frac{2}{\varepsilon^{2}} W\left(W+b_{\varepsilon}^{\infty}\right) \quad \text { on } \Omega .
$$

If $x_{0} \in K$ and $R:=\operatorname{dist}\left(x_{0}, \partial \Omega\right)$, the rescaled function

$$
Y(x):=W\left(R\left(x-x_{0}\right)\right)
$$

is thus a subsolution to the equation

$$
\Delta y=\frac{2}{\tilde{\varepsilon}^{2}} y\left(y+b_{\varepsilon}^{\infty}\right) \quad \text { on } B(0,1),
$$

where $\tilde{\varepsilon}:=\varepsilon / R$. On the other hand, it is easy to check that there exists a constant $C>0$ depending only on $N$ such that the function

$$
Z(x):= \begin{cases}C \tilde{\varepsilon}^{2}(|x|-1)^{-2} & \text { if }|x| \in\left[\frac{1}{3}, 1\right], \\ \frac{9}{8} C \tilde{\varepsilon}^{2}+\frac{81}{8} C \tilde{\varepsilon}^{2}|x|^{2} & \text { if }|x| \in\left[0, \frac{1}{3}\right],\end{cases}
$$

is a supersolution to $\mathrm{A}-2$ [notice that $Y(x) \rightarrow \infty$ as $|x| \rightarrow 1$ ]. It then follows from the maximum principle that $Y(x) \leq Z(x)$ for all $x$ in $B(0,1)$, and in particular

$$
W\left(x_{0}\right)=Y(0) \leq C \tilde{\varepsilon}^{2} \leq C \frac{\varepsilon^{2}}{\operatorname{dist}(K, \partial \Omega)^{2}} .
$$

Hence, we obtain the desired estimate

$$
\|w\|_{L^{\infty}(K)} \leq\|W\|_{L^{\infty}(K)}+b_{\varepsilon}^{\infty} \leq 1+c_{\infty}^{2} \varepsilon^{2}|\log \varepsilon|^{2}+C \frac{\varepsilon^{2}}{\operatorname{dist}(K, \partial \Omega)^{2}} .
$$

Concerning the estimate on the gradient, let $r:=\operatorname{dist}(K, \partial \Omega)$ and

$$
\tilde{K}:=\{x \in \Omega: \operatorname{dist}(x, K) \leq r / 2\} .
$$

By the first step, $\|w\|_{L^{\infty}(\tilde{K})} \leq C_{K}$, where $C_{K}$ does not depend on $w$ or $\varepsilon$. Let $U$ be the solution of

$$
\begin{cases}\left(\nabla-i|\log \varepsilon| \frac{\mathbf{c}}{2}\right)^{2} U=0 & \text { on } \tilde{K} \\ U=w & \text { on } \partial \tilde{K} .\end{cases}
$$


Since $w-U \in H^{2}(\tilde{K}) \cap H_{0}^{1}(\tilde{K})$, we deduce from the Gagliardo-Nirenberg inequality that

$$
\begin{aligned}
\|\nabla(w-U)\|_{L^{\infty}(\tilde{K})} & \leq\|\Delta(w-U)\|_{L^{\infty}(\tilde{K})}^{1 / 2} \cdot\|w-U\|_{L^{\infty}(\tilde{K})}^{1 / 2} \\
& \leq C_{K}\left(\frac{1}{\varepsilon}+|\log \varepsilon|^{1 / 2} c_{\infty}^{1 / 2}\|\nabla(w-U)\|_{L^{\infty}(\tilde{K})}^{1 / 2}\right) \\
& \leq C_{K}\left(\frac{1}{\varepsilon}+\frac{1}{2 C_{K}}\|\nabla(w-U)\|_{L^{\infty}(\tilde{K})}+2 C_{K} c_{\infty}|\log \varepsilon|\right)
\end{aligned}
$$

so that

$$
\|\nabla(w-U)\|_{L^{\infty}(\tilde{K})} \leq \frac{C_{K}}{\varepsilon} .
$$

Hence, since $U$ satisfies $\mathrm{A}-3$

$$
\|\nabla w\|_{L^{\infty}(K)} \leq\|\nabla U\|_{L^{\infty}(K)}+\frac{C_{K}}{\varepsilon} \leq \frac{C_{K}}{\varepsilon}\|U\|_{L^{\infty}(\tilde{K})}+\frac{C_{K}}{\varepsilon} \leq \frac{C_{K}}{\varepsilon},
$$

where $C_{K}$ depends only on $K, N$ and $c_{\infty}$. The lemma is proved.

Let us now define the 2-forms on $\mathbb{R}^{N}$,

$$
\xi_{j}(x):=\frac{2}{N-1} \sum_{i \neq j} x_{i} d x_{j} \wedge d x_{i} \quad \text { for } j=1, \ldots, N,
$$

which satisfy the equations $d^{*} \xi_{j}=2 d x_{j}$.

Lemma A.2 (Pohozaev identity). Let $w$ be a solution of equation (124) on $\Omega$. Then

$$
\begin{gathered}
\frac{N-2}{2} \int_{\Omega}|\nabla w|^{2}+\frac{N}{4 \varepsilon^{2}} \int_{\Omega}\left(a_{\varepsilon}(x)-|w|^{2}\right)^{2}-\frac{N-1}{2}|\log \varepsilon| \int_{\Omega}\left\langle J w, \sum_{i} c_{i}(x) \xi_{i}(x)\right\rangle \\
=\int_{\partial \Omega}\left[x \cdot v \frac{|\nabla w|^{2}}{2}+\frac{x \cdot v}{4 \varepsilon^{2}}\left(a_{\varepsilon}(x)-|w|^{2}\right)^{2}-\frac{\partial w}{\partial v} \cdot\left(\sum x_{i} \frac{\partial w}{\partial x_{i}}\right)\right] \\
+\frac{1}{2}|\log \varepsilon|^{2} \int_{\Omega}\left(a_{\varepsilon}(x)-|w|^{2}\right) x \cdot \nabla d(x) .
\end{gathered}
$$

In particular, for $B_{r}\left(x_{0}\right) \subset \Omega$ we have

$$
\begin{aligned}
& \frac{N-2}{2} \int_{B_{r}\left(x_{0}\right)}|\nabla w|^{2}+\frac{N}{4 \varepsilon^{2}} \int_{B_{r}\left(x_{0}\right)}\left(a_{\varepsilon}(x)-|w|^{2}\right)^{2} \\
& =\frac{N-1}{2}|\log \varepsilon| \int_{B_{r}\left(x_{0}\right)}\left\langle J w, \sum_{i} c_{i}(x) \xi_{i}\left(x-x_{0}\right)\right\rangle \\
& \quad+\frac{1}{2}|\log \varepsilon|^{2} \int_{B_{r}\left(x_{0}\right)}\left(a_{\varepsilon}(x)-|w|^{2}\right)\left(x-x_{0}\right) \cdot \nabla d(x) \\
& +\int_{\partial B_{r}\left(x_{0}\right)}\left[r \frac{\left|\nabla_{\top} w\right|^{2}}{2}-\frac{r}{2}\left|\frac{\partial w}{\partial n}\right|^{2}+\frac{r}{4 \varepsilon^{2}}\left(a_{\varepsilon}(x)-|w|^{2}\right)^{2}\right] .
\end{aligned}
$$


For $x_{0} \in \Omega$ and $r>0$ such that $B_{r}\left(x_{0}\right) \subset \Omega$, consider the scaled energy

$$
\tilde{E}_{\varepsilon}\left(w, x_{0}, r\right):=\frac{1}{r^{N-2}} E_{\varepsilon}\left(w, x_{0}, r\right) \equiv \frac{1}{r^{N-2}} \int_{B_{r}\left(x_{0}\right)}\left(\frac{1}{2}|\nabla w|^{2}+\frac{\left(a_{\varepsilon}(x)-|w|^{2}\right)^{2}}{4 \varepsilon^{2}}\right) .
$$

When this will not lead to confusion, we will also denote it by $\tilde{E}_{\varepsilon}\left(x_{0}, r\right)$ or even $\tilde{E}_{\varepsilon}(r)$.

Lemma A.3. Let $w$ satisfy 124 on $B_{R}\left(x_{0}\right) \subset \Omega$. Then for $0<r<R$,

$$
\begin{aligned}
\frac{d}{d r} \tilde{E}_{\varepsilon}\left(x_{0}, r\right)= & \frac{1}{r^{N-2}} \int_{\partial B_{r}\left(x_{0}\right)}\left|\frac{\partial w}{\partial n}\right|^{2}+\frac{1}{r^{N-1}} \int_{B_{r}\left(x_{0}\right)} \frac{\left(a_{\varepsilon}(x)-|w|^{2}\right)^{2}}{2 \varepsilon^{2}} \\
& -\frac{N-1}{2 r^{N-1}}|\log \varepsilon| \int_{B_{r}\left(x_{0}\right)}\left\langle J w, \sum_{i} c_{i}(x) \xi_{i}\left(x-x_{0}\right)\right\rangle \\
& -\frac{1}{2 r^{N-1}}|\log \varepsilon|^{2} \int_{B_{r}\left(x_{0}\right)}\left(\left(x-x_{0}\right) \cdot \nabla d(x)\right)\left(a_{\varepsilon}(x)-|w|^{2}\right) .
\end{aligned}
$$

Proof. Without loss of generality, we can assume that $x_{0}=0$. First one has

$$
\begin{aligned}
\frac{d}{d r} E_{\varepsilon}(r) & =\int_{\partial B_{r}} \frac{|\nabla w|^{2}}{2}+\frac{1}{4 \varepsilon^{2}} \int_{\partial B_{r}}\left(a_{\varepsilon}(x)-|w|^{2}\right)^{2} \\
& =\int_{\partial B_{r}}\left(\frac{|\nabla \top w|^{2}}{2}+\frac{1}{2}\left|\frac{\partial w}{\partial n}\right|^{2}+\frac{1}{4 \varepsilon^{2}}\left(a_{\varepsilon}(x)-|w|^{2}\right)^{2}\right) .
\end{aligned}
$$

Hence,

$$
\begin{aligned}
\frac{d}{d r} \tilde{E}_{\varepsilon}(r)= & -\frac{N-2}{r^{N-1}} E_{\varepsilon}(r)+\frac{1}{r^{N-2}} \int_{\partial B_{r}}\left(\frac{\left|\nabla_{\top} w\right|^{2}}{2}+\frac{1}{2}\left|\frac{\partial w}{\partial n}\right|^{2}+\frac{\left(a_{\varepsilon}(x)-|w|^{2}\right)^{2}}{4 \varepsilon^{2}}\right) \\
= & -\left(\frac{N-2}{r^{N-1}} \int_{B_{r}} \frac{|\nabla w|^{2}}{2}+\frac{N-2}{4 \varepsilon^{2} r^{N-1}} \int_{B_{r}}\left(a_{\varepsilon}(x)-|w|^{2}\right)^{2}\right) \\
& +\frac{1}{r^{N-2}} \int_{\partial B_{r}}\left(\frac{\left|\nabla_{\top} w\right|^{2}}{2}+\frac{1}{2}\left|\frac{\partial w}{\partial n}\right|^{2}+\frac{1}{4 \varepsilon^{2}}\left(a_{\varepsilon}(x)-|w|^{2}\right)^{2}\right) \\
= & -\frac{1}{r^{N-1}}\left[\int_{B_{r}} \frac{N-2}{2}|\nabla w|^{2}+\frac{N}{4 \varepsilon^{2}} \int_{B_{r}}\left(a_{\varepsilon}(x)-|w|^{2}\right)^{2}\right] \\
& +\frac{1}{2 \varepsilon^{2} r^{N-1}} \int_{B_{r}}\left(a_{\varepsilon}(x)-|w|^{2}\right)^{2} \\
& +\frac{1}{r^{N-2}} \int_{\partial B_{r}}\left(\frac{\left|\nabla_{\top} w\right|^{2}}{2}+\frac{1}{2}\left|\frac{\partial w}{\partial n}\right|^{2}+\frac{1}{4 \varepsilon^{2}}\left(a_{\varepsilon}(x)-|w|^{2}\right)^{2}\right) .
\end{aligned}
$$


Using Lemma A.2, we obtain

$$
\begin{aligned}
\frac{d}{d r} \tilde{E}_{\varepsilon}(r)= & -\frac{1}{r^{N-2}} \int_{\partial B_{r}}\left(\frac{\left|\nabla_{\top} w\right|^{2}}{2}-\frac{1}{2}\left|\frac{\partial w}{\partial n}\right|^{2}+\frac{1}{4 \varepsilon^{2}}\left(a_{\varepsilon}(x)-|w|^{2}\right)^{2}\right) \\
& -\frac{N-1}{2 r^{N-1}}|\log \varepsilon| \int_{B_{r}}\left\langle J w, \sum_{i} c_{i}(x) \xi_{i}(x)\right\rangle \\
& -\frac{1}{2 r^{N-1}}|\log \varepsilon|^{2} \int_{B_{r}}(x \cdot \nabla d(x))\left(a_{\varepsilon}(x)-|w|^{2}\right) \\
& +\frac{1}{r^{N-2}} \int_{\partial B_{r}}\left(\frac{\left|\nabla_{\top} w\right|^{2}}{2}+\frac{1}{2}\left|\frac{\partial w}{\partial n}\right|^{2}+\frac{1}{4 \varepsilon^{2}}\left(a_{\varepsilon}(x)-|w|^{2}\right)^{2}\right) \\
= & \frac{1}{r^{N-2}} \int_{\partial B_{r}}\left|\frac{\partial w}{\partial n}\right|^{2}+\frac{1}{r^{N-1}} \int_{B_{r}} \frac{\left(a_{\varepsilon}(x)-|w|^{2}\right)^{2}}{2 \varepsilon^{2}} \\
& -\frac{N-1}{2 r^{N-1}}|\log \varepsilon| \int_{B_{r}}\left\langle J w, \sum_{i} c_{i}(x) \xi_{i}(x)\right\rangle \\
& -\frac{1}{2 r^{N-1}}|\log \varepsilon|^{2} \int_{B_{r}}(x \cdot \nabla d(x))\left(a_{\varepsilon}(x)-|w|^{2}\right),
\end{aligned}
$$

which yields the result.

Proof of Lemma 4(Monotonicity at small scales). Again we can assume that $x_{0}=0$. In view of the previous lemma, we need to estimate the last two terms in $(\mathrm{A}-8)$. For the first one, notice that

$$
\|J w(x)\| \leq C|\nabla w(x)|^{2} \quad \text { and } \quad\left\|\xi_{j}(x)\right\| \leq C r \quad \text { for all } x \in B_{r},
$$

where $\|\cdot\|$ refers e.g. to the Euclidean norm on 2-forms. Hence,

$$
\begin{aligned}
& \frac{N-1}{2 r^{N-1}}|\log \varepsilon|\left|\int_{B_{r}}\left\langle J w, \sum_{i} c_{i}(x) \xi_{i}(x)\right\rangle\right| \\
& \quad \leq C c_{\infty}|\log \varepsilon| \frac{1}{r^{N-2}} \int_{B_{r}}|\nabla w|^{2} \leq C c_{\infty}|\log \varepsilon| \tilde{E}_{\varepsilon}(r),
\end{aligned}
$$

where $C$ depends only on $N$. For the second term we have

$$
\begin{aligned}
& \frac{1}{2 r^{N-1}}|\log \varepsilon|^{2}\left|\int_{B_{r}}(x \cdot \nabla d(x))\left(a_{\varepsilon}(x)-|w|^{2}\right)\right| \\
& \leq \frac{C}{r^{N-2}} \varepsilon|\log \varepsilon|^{2}\left(\int_{B_{r}}|\nabla d|^{2}\right)^{1 / 2} \cdot\left(\int_{B_{r}} \frac{\left(a_{\varepsilon}(x)-|w|^{2}\right)^{2}}{4 \varepsilon^{2}}\right)^{1 / 2} \\
& \leq C \Lambda_{0} r^{(2-N) / 2} \varepsilon|\log \varepsilon|^{2}\left(\int_{B_{r}} \frac{\left(a_{\varepsilon}(x)-|w|^{2}\right)^{2}}{4 \varepsilon^{2}}\right)^{1 / 2} \\
& \leq C \Lambda_{0} \varepsilon|\log \varepsilon|^{2} \tilde{E}_{\varepsilon}(r)^{1 / 2} \leq \tilde{E}_{\varepsilon}(r)+C^{2} \Lambda_{0}^{2} \varepsilon^{2}|\log \varepsilon|^{4} .
\end{aligned}
$$

Set $\Lambda:=C\left(c_{\infty}+1\right)|\log \varepsilon|$. Then by Lemma A.3, A-9] and A-10, 


$$
\begin{aligned}
& \frac{d}{d r}\left(\exp (\Lambda r) \tilde{E}_{\varepsilon}(r)\right)=\Lambda \exp (\Lambda r) \tilde{E}_{\varepsilon}(r)+\exp (\Lambda r) \frac{d}{d r} \tilde{E}_{\varepsilon}(r) \\
& \quad \geq \Lambda \exp (\Lambda r) \tilde{E}_{\varepsilon}(r)-\exp (\Lambda r)\left(C c_{\infty}|\log \varepsilon| \tilde{E}_{\varepsilon}(r)+\tilde{E}_{\varepsilon}(r)+C^{2} \Lambda_{0}^{2} \varepsilon^{2}|\log \varepsilon|^{4}\right) \\
& \left.\quad \geq-\exp (\Lambda r) C^{2} \Lambda_{0}^{2} \varepsilon^{2}|\log \varepsilon|^{4}\right)=-\frac{d}{d r}\left(\frac{Q^{2}}{\Lambda} \exp (\Lambda r)\right)
\end{aligned}
$$

This finishes the proof.

As already mentioned, the pointwise estimate on the Jacobian used in the previous proof is far from being optimal. In order to obtain a monotonicity formula valid on larger balls, we will use the following estimate due to Jerrard and Soner [29] (see [29] for a more quantitative version).

Lemma A.4 (Jerrard \& Soner). Let $w \in H_{\text {loc }}^{1}(\Omega, \mathbb{C}), \varphi \in \mathcal{C}_{\mathrm{c}}^{0,1}\left(\Omega, \Lambda^{2} \mathbb{R}^{N}\right)$ and set $K:=\operatorname{supp} \varphi$. Then there exist constants $C>0$ (depending only on $N)$ and $0<\alpha<1$ such that

$$
\begin{aligned}
\left|\int_{\Omega}\langle J w, \varphi\rangle\right| \leq & \frac{C}{|\log \varepsilon|}\|\varphi\|_{L^{\infty}} \int_{K} e_{\varepsilon}(w) \\
& +C \varepsilon^{\alpha}\|d \varphi\|_{L^{\infty}}\left(1+\int_{K} e_{\varepsilon}(w)\right)\left(1+|K|^{2}\right) .
\end{aligned}
$$

The big advantage of $\mathrm{A}-12$ with respect to estimate $\mathrm{A}-9]$ is the factor $1 /|\log \varepsilon|$ which appears in front of the energy. However, since A-12 contains a second term involving a derivative of $\varphi$, we need to adapt temporarily the definition of $\tilde{E}_{\varepsilon}$.

We define a cut-off function $f$ on $\mathbb{R}_{+} \times \mathbb{R}_{+}$by

$$
f(a, b)= \begin{cases}1 & \text { if } b \leq a \\ 2-b / a & \text { if } a \leq b \leq 2 a \\ 0 & \text { if } b \geq 2 a\end{cases}
$$

For $x_{0} \in \Omega$ and $r>0$ such that $B_{2 r}\left(x_{0}\right) \subset \Omega$, we will consider the quantity

$$
\bar{E}_{\varepsilon}\left(x_{0}, r\right):=\frac{1}{r^{N-2}} \int_{B_{2 r}\left(x_{0}\right)} e_{\varepsilon}(w) f\left(r,\left|x-x_{0}\right|\right) d x .
$$

Lemma A.5. Let $w$ satisfy (124) on $B_{R}\left(x_{0}\right) \subset \Omega$. Then for $0<r<R / 2$,

$$
\begin{aligned}
\frac{d}{d r} \bar{E}_{\varepsilon}\left(x_{0}, r\right) & \\
= & \frac{1}{r^{N-2}} \int_{1}^{2} t \int_{\partial B_{t r}\left(x_{0}\right)}\left|\frac{\partial w}{\partial n}\right|^{2}+\frac{1}{r^{N-1}} \int_{B_{2 r}\left(x_{0}\right)} \frac{\left(a_{\varepsilon}(x)-|w|^{2}\right)^{2}}{2 \varepsilon^{2}} f\left(r,\left|x-x_{0}\right|\right) \\
& -\frac{N-1}{2 r^{N-1}}|\log \varepsilon| \int_{B_{2 r}\left(x_{0}\right)}\left\langle J w, \sum_{i} c_{i}(x) \xi_{i}\left(x-x_{0}\right) f\left(r,\left|x-x_{0}\right|\right)\right\rangle \\
& -\frac{1}{2 r^{N-1}}|\log \varepsilon|^{2} \int_{B_{2 r}\left(x_{0}\right)}\left(\left(x-x_{0}\right) \cdot \nabla d(x)\right)\left(a_{\varepsilon}(x)-|w|^{2}\right) f\left(r,\left|x-x_{0}\right|\right) .
\end{aligned}
$$


Proof. For $x_{0}=0$ we have

$$
\begin{aligned}
\frac{d}{d r} \bar{E}_{\varepsilon}(r) & =-\frac{N-2}{r^{N-1}} \int_{B_{2 r}} e_{\varepsilon}(w) f(r,|x|) d x+\frac{1}{r^{N-2}} \int_{B_{2 r}} e_{\varepsilon}(w) \partial_{r} f(r,|x|) d x \\
& =-\frac{N-2}{r^{N-1}} \int_{1}^{2} \int_{B_{t r}} e_{\varepsilon}(w) d x d t+\frac{1}{r^{N-2}} \int_{r}^{2 r} \int_{\partial B_{t}} e_{\varepsilon}(w) \frac{t}{r^{2}} d x d t \\
& =-\frac{N-2}{r^{N-1}} \int_{1}^{2} \int_{B_{t r}} e_{\varepsilon}(w) d x d t+\frac{1}{r^{N-2}} \int_{1}^{2} t \int_{\partial B_{t r}} e_{\varepsilon}(w) d x d t \\
& =\int_{1}^{2} t^{N-1} \frac{d}{d(t r)} \tilde{E}_{\varepsilon}(t r) d t .
\end{aligned}
$$

It then suffices to use Lemma A.3 and to integrate in $t$. The case $x_{0} \neq 0$ is reduced to the first one by a change of variable.

Lemma A.6 (Monotonicity at large scales). There exists a constant $C>0$ such that for any $w$ satisfying 124 and $x_{0} \in \Omega, r>0$ such that $B_{2 r}\left(x_{0}\right) \subset \Omega$,

$$
\bar{E}_{\varepsilon}\left(\theta r, x_{0}\right) \leq C \exp \left(C \Lambda_{0} r\right)\left(\bar{E}_{\varepsilon}\left(r, x_{0}\right)+\frac{\varepsilon^{\alpha}|\log \varepsilon|}{(\theta r)^{N-1}}+\Lambda_{0} \varepsilon^{2}|\log \varepsilon|^{4}\right)
$$

for every $0<\theta \leq 1$.

Proof. The proof bears some resemblance to the one of Lemma 4. Once more we restrict ourselves to the case $x_{0}=0$; we first need to estimate the last two terms in $(\mathrm{A}-14)$. The second one is treated as before:

$$
\begin{aligned}
& \frac{1}{2 r^{N-1}}|\log \varepsilon|^{2} \int_{B_{2 r}}(x \cdot \nabla d(x))\left(a_{\varepsilon}(x)-|w|^{2}\right) f(r,|x|) \\
& \quad \leq \frac{C}{r^{N-2}} \varepsilon|\log \varepsilon|^{2}\left(\int_{B_{2 r}}|\nabla d|^{2}\right)^{1 / 2} \cdot\left(\int_{B_{2 r}} \frac{\left(a_{\varepsilon}(x)-|w|^{2}\right)^{2}}{4 \varepsilon^{2}}\right)^{1 / 2} \\
& \leq C \Lambda_{0} r^{(2-N) / 2} \varepsilon|\log \varepsilon|^{2}\left(\int_{B_{2 r}} \frac{\left(a_{\varepsilon}(x)-|w|^{2}\right)^{2}}{4 \varepsilon^{2}}\right)^{1 / 2} \\
& \leq C \Lambda_{0} \varepsilon|\log \varepsilon|^{2} \bar{E}_{\varepsilon}(2 r)^{1 / 2} \leq \bar{E}_{\varepsilon}(2 r)+C^{2} \Lambda_{0}^{2} \varepsilon^{2}|\log \varepsilon|^{4} .
\end{aligned}
$$

Concerning the first term, notice that the 2-form

$$
\varphi(x):=\sum_{i} c_{i}(x) \xi_{i}(x) f(r,|x|)
$$

satisfies the bounds

$$
\|\varphi\|_{L^{\infty}\left(B_{2 r}\right)} \leq C c_{\infty} r, \quad\|d \varphi\|_{L^{\infty}\left(B_{2 r}\right)} \leq C \Lambda_{0} .
$$


Hence, using Lemma A.4, we obtain

$$
\begin{aligned}
\frac{N-1}{2 r^{N-1}}|\log \varepsilon| \int_{B_{2 r}}\langle J w, & \left.\sum_{i} c_{i}(x) \xi_{i}(x) f(r,|x|)\right\rangle \\
& \leq C c_{\infty} \bar{E}_{\varepsilon}(2 r)+C \Lambda_{0} \varepsilon^{\alpha}|\log \varepsilon|\left(\frac{1}{r^{N-1}}+\bar{E}_{\varepsilon}(2 r)\right) \\
& \leq C \Lambda_{0} \bar{E}_{\varepsilon}(2 r)+\frac{C \Lambda_{0}}{r^{N-1}} \varepsilon^{\alpha}|\log \varepsilon| .
\end{aligned}
$$

From (A-14), A-16 and (A-17) we thus infer that

$$
\frac{d}{d r} \bar{E}_{\varepsilon}(r) \geq-C \Lambda_{0} \bar{E}_{\varepsilon}(2 r)-C\left(\frac{\Lambda_{0}}{r^{N-1}} \varepsilon^{\alpha}|\log \varepsilon|+\Lambda_{0}^{2} \varepsilon^{2}|\log \varepsilon|^{4}\right) .
$$

The conclusion then follows from a discrete version of Gronwall's lemma given hereafter.

Lemma A.7 (Discrete Gronwall inequality). Let $h:(0,1] \rightarrow \mathbb{R}_{+}$be continuously differentiable and such that

$$
\begin{cases}h(s) \leq \theta^{N-2} h(\theta s) & \text { for all } \theta \in[1,2], \\ h^{\prime}(s) \geq-C h(2 s)-D & \text { for all } s \leq 1 / 2,\end{cases}
$$

where $C$ and $D$ are positive constants. Then

$$
h(s) \leq 2^{N-2} \exp (C t)(h(t)+D / C) \quad \text { for all } 0<s<t<1 .
$$

Proof. Let $g(s):=h(s)+D / C$. We have

$$
\begin{cases}g(s)=h(s)+\frac{D}{C} \leq \theta^{N-2} h(\theta s)+\theta^{N-2} \frac{D}{C}=\theta^{N-2} g(\theta s) & \text { for all } \theta \in[1,2], \\ g^{\prime}(s)=h^{\prime}(s) \geq-C h(2 s)-D=-C g(2 s) & \text { for all } s \leq 1 / 2,\end{cases}
$$

so that we just need to consider the case $D=0$. Let $0<s<t<1$ be given. If $s \in[t / 2, t]$, then by $\mathrm{A}-20$,

$$
g(s) \leq 2^{N-2} g(t) .
$$

By induction, assume that for some $k \in \mathbb{N}_{*}$,

$$
g(s) \leq 2^{N-2} g(t) \prod_{i=2}^{k}\left(1+\frac{C t}{2^{i}}\right) \quad \forall s \in\left[\frac{t}{2^{k}}, \frac{t}{2^{k-1}}\right] .
$$

Then, for $s \in\left[t / 2^{k+1}, t / 2^{k}\right]$,

$$
\begin{aligned}
g(s) & \leq g\left(\frac{t}{2^{k}}\right)+C \int_{s}^{t / 2^{k}} g(2 r) d r \\
& \leq 2^{N-2} g(t) \prod_{i=2}^{k}\left(1+\frac{C t}{2^{i}}\right)+\frac{C t}{2^{k+1}} 2^{N-2} \prod_{i=2}^{k}\left(1+\frac{C t}{2^{i}}\right) \\
& =2^{N-2} g(t) \prod_{i=2}^{k+1}\left(1+\frac{C t}{2^{i}}\right) .
\end{aligned}
$$


The conclusion then follows by using the fact that

$$
\prod_{i=1}^{m}\left(1+\frac{C t}{2^{i}}\right) \leq \exp (C t) \quad \text { for all } m \in \mathbb{N}_{*} .
$$

Coming back to $h$, we obtain

$$
h(s) \leq g(s) \leq 2^{N-2} \exp (C t) g(t)=2^{N-2} \exp (C t)(h(t)+D / C),
$$

and the proof is complete.

Notice that whereas Lemma 4 was appropriate for balls of radius of the order of $1 /|\log \varepsilon|$, Lemma A.6 is only appropriate for balls of radius larger than $O\left(\varepsilon^{\alpha /(N-1)}\right)$. This is caused by the oscillation term of order $\varepsilon^{\alpha} / r^{N-1}$. Fortunately, these two conditions complement perfectly to obtain Proposition 2 .

Proof of Proposition 2. We first consider the case

$$
\theta r<\rho:=\left(|\log \varepsilon|\left(c_{\infty}+1\right)\right)^{-1}<r / 2,
$$

the other ones being easier to treat. Using Lemma 4, we deduce that

$$
\tilde{E}_{\varepsilon}(\theta r) \leq C\left(\tilde{E}_{\varepsilon}(\rho)+\Lambda_{0}|\log \varepsilon|^{3} \varepsilon^{2}\right) .
$$

Next, by Lemma A.6 and the definition of $\rho$,

$$
\begin{aligned}
\tilde{E}_{\varepsilon}(\rho) & \leq \bar{E}_{\varepsilon}(\rho) \leq C\left(\bar{E}_{\varepsilon}(r / 2)+\frac{\varepsilon^{\alpha}|\log \varepsilon|}{\rho^{N-1}}+\Lambda_{0}|\log \varepsilon|^{4} \varepsilon^{2}\right) \\
& \leq C\left(2^{N-2} \tilde{E}_{\varepsilon}(r)+\varepsilon^{\alpha}|\log \varepsilon|^{N}\left(c_{\infty}+1\right)^{N-1}+\Lambda_{0}|\log \varepsilon|^{4} \varepsilon^{2}\right) .
\end{aligned}
$$

It then suffices to take $\beta=\alpha / 2$ and combine $(\mathrm{A}-22$ and $(\mathrm{A}-23)$ to get the desired estimate (23). In the case $\theta r \geq \rho$ (resp. $r \leq \rho$ ), it suffices to use Lemma A.6 (resp. Lemma 4) to obtain (23) directly. This finishes the proof.

Proof of Theorem 2 Through a scaling, we first show that we can assume without loss of generality that $x_{0}=0, r=1$ and $\Lambda_{0} \leq 1$. Indeed, let

$$
u(x):=w_{\varepsilon}\left(r\left(x-x_{0}\right)\right)
$$

Then $u$ satisfies the equation

$$
\Delta u+\frac{1}{\tilde{\varepsilon}^{2}} u\left(1-|u|^{2}\right)=i \tilde{c} \cdot \nabla u|\log \tilde{\varepsilon}|+\tilde{d}|\log \tilde{\varepsilon}|^{2} u
$$

on $B(0,2)$, where $\tilde{\varepsilon}:=\varepsilon / r, \quad \tilde{c}(x):=\mathbf{c}\left(r\left(x-x_{0}\right)\right) r|\log \varepsilon| /|\log \tilde{\varepsilon}|$, and $\tilde{d}(x):=$ $d\left(r\left(x-x_{0}\right)\right) r^{2}|\log \varepsilon|^{2} /|\log \tilde{\varepsilon}|^{2}$. Since $r \geq \sqrt{\varepsilon}$, we have $|\log \varepsilon| \leq 2|\log \tilde{\varepsilon}|$ so that $\Lambda_{0}(\tilde{c}, \tilde{d})$ $\leq 1$. We conclude by noticing that $\tilde{E}_{\varepsilon}\left(w_{\varepsilon}, x_{0}, r\right)=\tilde{E}_{\tilde{\varepsilon}}(u, 0,1)$.

From now on, we thus assume that $x_{0}=0, r=1$ and $\Lambda_{0} \leq 1$. For the ease of presentation, we follow closely the lines of [10]. Let $0<\delta<1 / 32$ be a constant to be determined later (and depending only on $N$ ). In what follows we will denote by $C$ generic constants not depending on the choice of $\delta$. 
Part A: Choosing a "good" radius

Lemma A.8. Assume that $0<\varepsilon<\delta^{2(N-1) / \alpha}$. Then there exists some constant $C>0$ and a radius $r_{0} \in\left(\varepsilon^{\alpha /(2 N-2)}, 1\right)$ such that

$$
\begin{aligned}
& \frac{1}{r_{0}^{N-2}} \int_{B_{r_{0}}} \frac{\left(a_{\varepsilon}-|w|^{2}\right)^{2}}{2 \varepsilon^{2}} \leq C\left(\eta|\log \delta|+\varepsilon^{\beta}\right), \\
& \tilde{E}_{\varepsilon}\left(r_{0}\right)-2^{N-2} \tilde{E}_{\varepsilon}\left(\delta r_{0}\right) \leq C\left(\eta|\log \delta|+\varepsilon^{\beta}\right) .
\end{aligned}
$$

Proof. We will essentially make use of (A-14) together with a covering argument. First notice that

$$
r_{0}>\varepsilon^{\alpha /(2 N-2)} \quad \text { implies } \quad \frac{\varepsilon^{\alpha}}{r^{N-1}} \leq \varepsilon^{\beta} \quad \text { for } r \geq r_{0} .
$$

Hence, from A-14 and following the lines of Lemma A.6 we obtain

$$
\left|\frac{d}{d r} \bar{E}_{\varepsilon}(r)-A(r)\right| \leq C \bar{E}_{\varepsilon}(2 r)+C \varepsilon^{\beta},
$$

where

$$
A(r):=\frac{1}{r^{N-2}} \int_{1}^{2} t \int_{\partial B_{t r}}\left|\frac{\partial w}{\partial n}\right|^{2}+\frac{1}{r^{N-1}} \int_{B_{2 r}} \frac{\left(a_{\varepsilon}(x)-|w|^{2}\right)^{2}}{2 \varepsilon^{2}} f\left(r,\left|x-x_{0}\right|\right) .
$$

From $\mathrm{A}-25$ and the monotonicity formula of Proposition 2 we thus infer that

$$
\int_{\varepsilon^{\alpha /(2 N-2)}}^{1 / 4}\left(A(r)+C \bar{E}_{\varepsilon}(2 r)+C \varepsilon^{\beta}\right) d r \leq C\left(\eta|\log \varepsilon|+\varepsilon^{\beta}\right) .
$$

Let $k$ be the greatest integer such that $\varepsilon^{\alpha /(2 N-2)}(\delta / 4)^{-k} \leq 1 / 4$, and define the intervals

$$
I_{j}:=\left(\varepsilon^{\alpha /(2 N-2)}(\delta / 4)^{-j+1}, \varepsilon^{\alpha /(2 N-2)}(\delta / 4)^{-j}\right), \quad 1 \leq j \leq k .
$$

Clearly, these intervals are disjoint and $\bigcup_{j=1}^{k} I_{j} \subset\left(\varepsilon^{\alpha /(2 N-2)}, 1 / 4\right)$. Since

$$
k \geq C^{-1} \frac{|\log \varepsilon|}{|\log \delta|}
$$

we deduce from $\mathrm{A}-26$ that there exists some $j_{0} \in\{1, \ldots, k\}$ such that

$$
\int_{I_{j_{0}}}\left(A(r)+C \bar{E}_{\varepsilon}(2 r)+C \varepsilon^{\beta}\right) d r \leq C\left(\eta|\log \delta|+\varepsilon^{\beta}\right) .
$$

In particular, by the mean-value formula there exists some

$$
r_{0} \in\left(\frac{1}{2} \varepsilon^{\alpha /(2 N-2)}(\delta / 4)^{-j}, \varepsilon^{\alpha /(2 N-2)}(\delta / 4)^{-j}\right)
$$

such that

$$
\frac{1}{r_{0}^{N-2}} \int_{B_{r_{0}}} \frac{\left(a_{\varepsilon}(x)-|w|^{2}\right)^{2}}{2 \varepsilon^{2}} \leq C\left(\eta|\log \delta|+\varepsilon^{\beta}\right)
$$


which establishes the first claim. Notice that $\frac{\delta}{2} r_{0} \in I_{0}$, hence

$$
\begin{aligned}
\tilde{E}_{\varepsilon}\left(r_{0}\right)-2^{N-2} \tilde{E}_{\varepsilon}\left(\delta r_{0}\right) & \leq \bar{E}_{\varepsilon}\left(r_{0}\right)-\bar{E}_{\varepsilon}\left(\frac{\delta}{2} r_{0}\right) \\
& \leq \int_{I_{j_{0}}} A(r)+C \bar{E}_{\varepsilon}(2 r)+C \varepsilon^{\beta} d r \\
& \leq C\left(\eta|\log \delta|+\varepsilon^{\beta}\right) .
\end{aligned}
$$

The lemma is proved.

Part B: $\delta$-Energy decay

In this second part, we present an estimate valid for any solution $u$ of 124 with $\Lambda_{0} \leq 1$. We will apply it later in Part $\mathrm{C}$ to an appropriate dilation of $w$. Let $0<\gamma<1 / 8$ be a constant to be determined later.

Lemma A.9. There exist constants $\varepsilon_{N}>0$ (depending only on $\gamma$ and $N$ ) and $C>0$ such that for any $0<\varepsilon<\varepsilon_{N}$ and any solution $u$ of $(124)$ on $B(0,2)$ for some $\mathbf{c}$ and $d$ satisfying $\Lambda_{0}(\mathbf{c}, d) \leq 1$ we have

$$
\begin{aligned}
E_{\varepsilon}(\delta) \leq & C\left(\left(\gamma^{2}+\delta^{N}+\gamma^{-4} \int_{B_{1}} \frac{\left(a_{\varepsilon}-|u|^{2}\right)^{2}}{\varepsilon^{2}}\right) E_{\varepsilon}(1)\right. \\
& \left.+\gamma^{-4}\left(\int_{B_{1}} \frac{\left(a_{\varepsilon}-|u|^{2}\right)^{2}}{\varepsilon^{2}}+\varepsilon^{\beta}\right)\right) .
\end{aligned}
$$

Proof. The starting point is the identity

$$
4|u|^{2}|\nabla u|^{2}=4|u \times \nabla u|^{2}+\left.\left.|\nabla| u\right|^{2}\right|^{2},
$$

which holds for any map from $\mathbb{R}^{N}$ to $\mathbb{R}^{k}$; in the special case where $k=2$ and $\left|u\left(x_{0}\right)\right| \neq 0$, near $x_{0}$ we may write

and then

$$
u(x)=\rho \exp (i \varphi)
$$

$$
u \times \nabla u=\rho^{2} \nabla \varphi,
$$

i.e. $u \times \nabla u$ plays the role of the gradient of the phase. The advantage of the form $\mathrm{A}-29$ is that $u \times \nabla u$ is always globally well defined, while the phase need not be well defined when $u$ vanishes somewhere.

Since $u$ is a solution on $B(0,2)$, we infer from Lemma 3 that there exists $\varepsilon_{N}$ depending only on $N$ and $\gamma$ and $C>0$ such that if $0<\varepsilon<\varepsilon_{N}$ then

$$
\|u\|_{\infty} \leq 1+\gamma / 2, \quad\|\nabla u\|_{\infty} \leq C / \varepsilon \quad \text { in } B(0,1) .
$$

By the mean-value inequality, we may find some $r_{1} \in\left[\frac{1}{16}, \frac{1}{8}\right]$ such that

$$
\begin{gathered}
\int_{\partial B_{r_{1}}}|\nabla u|^{2} \leq 32 \int_{B_{1}}|\nabla u|^{2}, \\
\int_{\partial B_{r_{1}}}\left(a_{\varepsilon}-|u|^{2}\right)^{2} \leq 32 \int_{B_{1}}\left(a_{\varepsilon}-|u|^{2}\right)^{2} .
\end{gathered}
$$

We divide the estimate into several steps. 
Step 1: Hodge-de Rham decomposition of $u \times \nabla u$. Observe that since $u$ is a solution of (124),

$$
\begin{aligned}
d^{*}(u \times d u) & =u \times \Delta u=(u, \mathbf{c} \cdot \nabla u)|\log \varepsilon| \\
& =d^{*}\left(\left(|u|^{2}-1\right) \sum c_{i}(x) d x_{i}|\log \varepsilon|\right) .
\end{aligned}
$$

Let $\xi$ be the solution of the auxiliary Neumann problem

$$
\begin{cases}\Delta \xi=0 & \text { in } B_{r_{1}} \\ \frac{\partial \xi}{\partial n}=u \times \frac{\partial u}{\partial n}-\left(|u|^{2}-1\right) \mathbf{c} \cdot \mathbf{n}|\log \varepsilon| & \text { on } \partial B_{r_{1}}\end{cases}
$$

Notice that $\xi$ exists since $\operatorname{div}\left(u \times \nabla u-\left(|u|^{2}-1\right) \mathbf{c}|\log \varepsilon|\right)=0$ implies by integration $\int_{\partial B_{r_{1}}}\left(u \times \nabla u-\left(|u|^{2}-1\right) \mathbf{c}|\log \varepsilon|\right) \cdot n=0$. Moreover, we have

$$
\int_{B_{r_{1}}}|\nabla \xi|^{2} \leq C \int_{B_{1}}|\nabla u|^{2}+C \varepsilon^{2}|\log \varepsilon|^{2} \int_{B_{1}} \frac{1-|u|^{2}}{\varepsilon^{2}} \leq C\left(E_{\varepsilon}(1)+\varepsilon^{\beta}\right) .
$$

Since $\xi$ is harmonic on $B_{r_{1}}$, we have by standard elliptic estimates, for $0<\delta \leq r_{1}$,

$$
\int_{B_{\delta}}|\nabla \xi|^{2} \leq C \delta^{N} \int_{B_{r_{1}}}|\nabla \xi|^{2} \leq C \delta^{N}\left(E_{\varepsilon}(1)+\varepsilon^{\beta}\right) .
$$

By construction we verify that

$$
d^{*}\left[\left(u \times d u-\left(|u|^{2}-1\right) \sum c_{i}(x) d x_{i}|\log \varepsilon|-d \xi\right) 1_{B_{r_{1}}}\right]=0 \quad \text { in } \mathcal{D}^{\prime}\left(\mathbb{R}^{N}\right),
$$

where $1_{A}$ denotes the characteristic function of the set $A$. By classical Hodge theory (see [10, Proposition A.7]) there exists some 2-form $\varphi$ on $\mathbb{R}^{N}$ such that $\varphi \in H_{\text {loc }}^{1}\left(\mathbb{R}^{N}\right)$ and

$$
\begin{aligned}
& d^{*} \varphi=\left(u \times d u-\left(|u|^{2}-1\right) \sum c_{i}(x) d x_{i}|\log \varepsilon|-d \xi\right) 1_{B_{r_{1}}} \text { in } \mathcal{D}^{\prime}\left(\mathbb{R}^{N}\right) \\
& d \varphi=0 \quad \text { in } \mathcal{D}^{\prime}\left(\mathbb{R}^{N}\right), \\
& \|\nabla \varphi\|_{L^{2}\left(\mathbb{R}^{N}\right)} \leq C\left(E_{\varepsilon}\left(r_{1}\right)+\|\nabla \xi\|_{L^{2}\left(B_{r_{1}}\right)}\right), \\
& |\varphi(x)| \cdot|x|^{N-1} \quad \text { tends to zero at infinity. }
\end{aligned}
$$

We therefore have

$$
u \times d u=d^{*} \varphi+d \xi+\left(|u|^{2}-1\right) \sum c_{i}(x) d x_{i}|\log \varepsilon| \quad \text { in } B_{r_{1}} .
$$

In order to bound the $L^{2}$-norm of $u \times d u$ on $B_{\delta}$, we next turn to estimates for $d^{*} \varphi$.

Step 2: Improved estimates for $\nabla \varphi$ on $B_{\delta}$. Let $f: \mathbb{R}^{+} \rightarrow(1,1 /(1-\gamma))$ be any smooth function such that

$$
\begin{cases}f(t)=1 / t & \text { if } t \geq 1-\gamma, \\ f(t)=1 & \text { if } t \leq 1-2 \gamma \\ \left|f^{\prime}(t)\right| \leq 4 & \text { for any } t \in \mathbb{R}^{+}\end{cases}
$$


Define on $\mathbb{R}^{N}$ the function $\tau$ by

$$
\tau(x)= \begin{cases}f^{2}(|u(x)|) & \text { in } B_{r_{1}}, \\ 1 & \text { outside, }\end{cases}
$$

so that, taking $\mathrm{A}-30 \mathrm{into}$ account,

$$
0 \leq \tau-1 \leq 4 \gamma \quad \text { in } \mathbb{R}^{N}
$$

Notice that

$$
f^{2}(|u|) u \times d u=f(|u|) u \times d(f(|u|) u),
$$

hence

$$
d(\tau u \times d u)=d\left(f^{2}(|u|) u \times d u\right)=d(f(|u|) u \times d(f(|u|) u)) \quad \text { in } B_{r_{1}},
$$

i.e.

$$
d(\tau u \times d u)=\sum_{i<j} 2(f(|u|) u)_{x_{i}} \times(f(|u|) u)_{x_{j}} d x_{i} \wedge d x_{j} .
$$

Now we turn to $\varphi$. We have

$$
\begin{aligned}
-\Delta \varphi=d d^{*} \varphi= & d\left(1_{B_{r_{1}}} \tau u \times d u\right)-d\left(1_{B_{r_{1}}} d \xi\right)-d\left(1_{B_{r_{1}}}\left(|u|^{2}-1\right) \sum c_{i} d x_{i}|\log \varepsilon|\right) \\
& +d\left(1_{B_{r_{1}}}(1-\tau) u \times d u\right) \quad \text { in } \mathcal{D}^{\prime}\left(\mathbb{R}^{N}\right) \\
= & \omega_{1}+\omega_{2}+\omega_{3}+\omega_{4}+\omega_{5},
\end{aligned}
$$

where

$$
\begin{aligned}
& \omega_{1}=1_{B_{r_{1}}} d(\tau u \times d u)=1_{B_{r_{1}}} \sum_{i<j} 2(f(|u|) u)_{x_{i}} \times(f(|u|) u)_{x_{j}} d x_{i} \wedge d x_{j}, \\
& \omega_{2}=\sigma_{\partial B_{r_{1}}} f(|u|) u \times d u \wedge d r \quad(r=|x|) \\
& \omega_{3}=-d\left(1_{B_{r_{1}}} d \xi\right)=\sigma_{\partial B_{r_{1}}} d r \wedge d \xi \\
& \omega_{4}=-d\left(1_{B_{r_{1}}}\left(|u|^{2}-1\right) \sum c_{i} d x_{i}|\log \varepsilon|\right) \\
& \omega_{5}=d\left(1_{B_{r_{1}}}(1-\tau) u \times d u\right)
\end{aligned}
$$

Here $\sigma_{\partial B_{r_{1}}}$ stands for the surface measure on $\partial B_{r_{1}}$. Set $\varphi_{i}:=G * \omega_{i}$, where $G(x):=$ $c_{N}|x|^{2-N}$ is the fundamental solution of $-\Delta$ in $\mathbb{R}^{N}$. Since $\varphi$ tends to zero at infinity by A-37) and each $\varphi_{i}$ tends to zero at infinity (because each $\omega_{i}$ has compact support), we conclude that

$$
\varphi=\sum_{i=1}^{5} \varphi_{i}
$$

We now proceed to estimate separately each $\varphi_{i}$.

Estimate for $\varphi_{5}$. We have

$$
\int_{\mathbb{R}^{N}}\left|\nabla \varphi_{5}\right|^{2} \leq C \gamma^{2} \int_{B_{1}}|\nabla u|^{2} .
$$


Indeed, we have

$$
-\Delta \varphi_{5}=\omega_{5}=d\left(1_{B_{r_{1}}}(1-\tau) u \times d u\right) .
$$

Multiplying by $\varphi_{5}$ and integrating we obtain

$$
\int_{\mathbb{R}^{N}}\left|\nabla \varphi_{5}\right|^{2} \leq\|1-\tau\|_{L^{\infty}\left(B_{1}\right)}\|u\|_{L^{\infty}\left(B_{1}\right)}\|\nabla u\|_{L^{2}\left(B_{1}\right)}\left\|\nabla \varphi_{5}\right\|_{L^{2}}
$$

and thus

$$
\int_{\mathbb{R}^{N}}\left|\nabla \varphi_{5}\right|^{2} \leq C \gamma\|\nabla u\|_{L^{2}\left(B_{1}\right)}\left\|\nabla \varphi_{5}\right\|_{L^{2}\left(\mathbb{R}^{N}\right)},
$$

by $(\mathrm{A}-30$ ) and $\mathrm{A}-39$, which yields the result.

Estimate for $\varphi_{4}$. We have

$$
\int_{\mathbb{R}^{N}}\left|\nabla \varphi_{4}\right|^{2} \leq C \int_{B_{1}} \frac{\left(1-|u|^{2}\right)^{2}}{\varepsilon^{2}} \leq C\left(\int_{B_{1}} \frac{\left(a_{\varepsilon}-|u|^{2}\right)^{2}}{\varepsilon^{2}}+\varepsilon^{\beta}\right) .
$$

Indeed, we have

$$
-\Delta \varphi_{4}=\omega_{4}=-d\left(1_{B_{r_{1}}}\left(|u|^{2}-1\right) \sum c_{i} d x_{i}|\log \varepsilon|\right) .
$$

Multiplying by $\varphi_{4}$ and integrating we obtain

$$
\int_{\mathbb{R}^{N}}\left|\nabla \varphi_{4}\right|^{2} \leq \varepsilon|\log \varepsilon| \cdot\|\mathbf{c}\|_{L^{\infty}\left(B_{1}\right)}\left(\int_{B_{1}} \frac{\left(1-|u|^{2}\right)^{2}}{\varepsilon^{2}}\right)^{1 / 2}\left\|\nabla \varphi_{4}\right\|_{L^{2}},
$$

which yields the result since $\Lambda_{0} \leq 1$.

Estimate for $\varphi_{3}$. We have

$$
\int_{B_{\delta}}\left|\nabla \varphi_{3}\right|^{2} \leq C \delta^{N}\left(E_{\varepsilon}(1)+\varepsilon^{\beta}\right) .
$$

Indeed, we have

$$
-\Delta \varphi_{3}=\omega_{3}=-d\left(1_{B_{r_{1}}} d \xi\right) .
$$

Multiplying by $\varphi_{3}$ and integrating we obtain

$$
\int_{\mathbb{R}^{N}}\left|\nabla \varphi_{3}\right|^{2} \leq\|\nabla \xi\|_{L^{2}\left(B_{r_{1}}\right)}\left\|\nabla \varphi_{3}\right\|_{L^{2}} .
$$

Since $\varphi_{3}$ is harmonic on $B_{r_{1}}\left(r_{1} \geq 1 / 16\right)$, we also have

$$
\left\|\nabla \varphi_{3}\right\|_{L^{\infty}\left(B_{1 / 32}\right)} \leq C\left\|\nabla \varphi_{3}\right\|_{L^{2}\left(B_{r_{1}}\right)},
$$

so that $(\delta \leq 1 / 32)$

$$
\int_{B_{\delta}}\left|\nabla \varphi_{3}\right|^{2} \leq C \delta^{N}\|\nabla \xi\|_{L^{2}\left(B_{r_{1}}\right)}^{2} \leq C \delta^{N}\left(E_{\varepsilon}(1)+\varepsilon^{\beta}\right) .
$$

Estimate for $\varphi_{2}$. We have

$$
\int_{B_{\delta}}\left|\nabla \varphi_{2}\right|^{2} \leq C \delta^{N} \int_{B_{1}}|\nabla u|^{2}
$$


Indeed, we have

$$
-\Delta \varphi_{2}=\omega_{2}=\sigma_{\partial B_{r_{1}}} f(|u|) u \times d u \wedge d r .
$$

By standard elliptic estimates for harmonic functions with measure data,

$$
\left\|\nabla \varphi_{2}\right\|_{L^{\infty}(B(1 / 32))} \leq C\left\|\omega_{2}\right\| \leq C\left(\int_{\partial B_{r_{1}}}|\nabla u|^{2}\right)^{1 / 2},
$$

so that using $\mathrm{A}-31 \mathrm{n}$ we finally obtain

$$
\int_{B_{\delta}}\left|\nabla \varphi_{2}\right|^{2} \leq C \delta^{N} \int_{B_{1}}|\nabla u|^{2}
$$

Estimate for $\varphi_{1}$. We start with the crucial observation that

$$
\left|\omega_{1}\right| \leq C \gamma^{-2} \frac{\left(a_{\varepsilon}-|u|^{2}\right)^{2}}{\varepsilon^{2}} \quad \text { in } B_{1} .
$$

Indeed, we have to distinguish the two regions

$$
V_{\gamma}=\left\{x \in B_{1}:|u(x)| \geq 1-\gamma\right\}, \quad W_{\gamma}=\left\{x \in B_{1}:|u(x)| \leq 1-\gamma\right\} .
$$

Recall that

$$
\omega_{1}=1_{B_{r_{1}}} d(\tau u \times d u)=1_{B_{r_{1}}} \sum_{i<j} 2(f(|u|) u)_{x_{i}} \times(f(|u|) u)_{x_{j}} d x_{i} \wedge d x_{j} .
$$

On $V_{\gamma}$ we have $f(|u(x)|)=1 /|u(x)|$ and therefore

$$
(f(|u|) u)_{x_{i}} \times(f(|u|) u)_{x_{j}}=0 \quad \text { for } i \neq j .
$$

On $W_{\gamma}$ we have, by A-30,

$$
\left|(f(|u|) u)_{x_{i}}\right| \leq C / \varepsilon,
$$

so that

$$
\left|\omega_{1}\right| \leq \frac{C}{\varepsilon^{2}}=\frac{C}{\varepsilon^{2}} \gamma^{-2} \gamma^{2} \leq \frac{C}{\varepsilon^{2}} \gamma^{-2}(1-|u|)^{2} \leq C \gamma^{-2} \frac{\left(1-|u|^{2}\right)^{2}}{\varepsilon^{2}} .
$$

Decreasing $\varepsilon_{N}$ if necessary, we have

$$
\left(1-|u|^{2}\right)^{2} \leq 2\left(a_{\varepsilon}-|u|^{2}\right)^{2} \quad \text { on } W_{\gamma},
$$

which yields (A-44).

The final crucial estimate is

$$
\left\|\varphi_{1}\right\|_{L^{\infty}\left(\mathbb{R}^{N}\right)} \leq \frac{C}{\gamma^{2}}\left(E_{\varepsilon}(0,1)+\varepsilon^{\beta}\right) .
$$

Indeed,

$$
\varphi_{1}(x)=\int_{\mathbb{R}^{N}} \frac{c_{N}}{|x-y|^{N-2}} \omega_{1}(y) d y=\int_{B_{r_{1}}} \frac{c_{N}}{|x-y|^{N-2}} \omega_{1}(y) d y,
$$


so that

$$
\left|\varphi_{1}(x)\right| \leq \frac{C}{\gamma^{2}} \int_{B_{r_{1}}} \frac{\left(a_{\varepsilon}-|u(y)|^{2}\right)^{2}}{\varepsilon^{2}|x-y|^{N-2}} d y .
$$

Assume $|x| \leq r_{1} \leq 1 / 8$. Since $B_{r_{1}} \subset B_{1 / 4}(x)$ we have

$$
\left|\varphi_{1}(x)\right| \leq \frac{C}{\gamma^{2}} \int_{B_{1 / 4}(x)} \frac{\left(a_{\varepsilon}-|u(y)|^{2}\right)^{2}}{\varepsilon^{2}|x-y|^{N-2}} d y .
$$

Next, we observe that

$$
\begin{aligned}
& \int_{B_{1 / 4}(x)} \frac{\left(a_{\varepsilon}-|u(y)|^{2}\right)^{2}}{\varepsilon^{2}|x-y|^{N-2}} d y=\int_{0}^{1 / 4} \frac{1}{r^{N-2}}\left(\int_{\partial B_{r}} \frac{\left(a_{\varepsilon}-|u|^{2}\right)^{2}}{\varepsilon^{2}}\right) d r \\
& =(N-2) \int_{0}^{1 / 4} \frac{1}{r^{N-1}}\left(\int_{B_{r}} \frac{\left(a_{\varepsilon}-|u|^{2}\right)^{2}}{\varepsilon^{2}}\right) d r+\left[\frac{1}{r^{N-2}} \int_{B_{r}} \frac{\left(a_{\varepsilon}-|u|^{2}\right)^{2}}{\varepsilon^{2}}\right]_{0}^{1 / 4} .
\end{aligned}
$$

Using the monotonicity formulae (A-8) when $r \in(0,1 /|\log \varepsilon|)$, and $(\mathrm{A}-14)$ when $r \in$ $(1 /|\log \varepsilon|, 1 / 4)$, together with the estimates in Lemmas 4 and A.6 we thus infer that

$$
\int_{B_{1 / 4}(x)} \frac{\left(a_{\varepsilon}-|u(y)|^{2}\right)^{2}}{\varepsilon^{2}|x-y|^{N-2}} d y \leq C\left(\tilde{E}_{\varepsilon}(x, 1 / 2)+\varepsilon^{\beta}\right) \leq C\left(E_{\varepsilon}(0,1)+\varepsilon^{\beta}\right),
$$

since $B(x, 1 / 2) \subset B(0,1)$. Hence for every $x \in B_{r_{1}}$

$$
\left|\varphi_{1}(x)\right| \leq C \gamma^{-2}\left(E_{\varepsilon}(0,1)+\varepsilon^{\beta}\right) .
$$

Recall that $\Delta \varphi_{1}=0$ outside $B_{r_{1}}$, so that by the maximum principle

$$
\left\|\varphi_{1}\right\|_{L^{\infty}\left(\mathbb{R}^{N}\right)}=\left\|\varphi_{1}\right\|_{L^{\infty}\left(B_{r_{1}}\right)} \leq C \gamma^{-2}\left(E_{\varepsilon}(0,1)+\varepsilon^{\beta}\right),
$$

which is $\mathrm{A}-45$.

Going back to the equation

$$
-\Delta \varphi_{1}=\omega_{1} \quad \text { in } \mathbb{R}^{N}
$$

we conclude that

$$
\int_{\mathbb{R}^{N}}\left|\nabla \varphi_{1}\right|^{2} \leq\left\|\varphi_{1}\right\|_{L^{\infty}\left(\mathbb{R}^{N}\right)} \int_{B_{r_{1}}}\left|\omega_{1}\right|
$$

so that

$$
\int_{\mathbb{R}^{N}}\left|\nabla \varphi_{1}\right|^{2} \leq C \gamma^{-4} \int_{B_{1}} \frac{\left(a_{\varepsilon}-|u|^{2}\right)^{2}}{\varepsilon^{2}}\left(E_{\varepsilon}(0,1)+\varepsilon^{\beta}\right) .
$$

We now gather the different estimates for $\varphi_{1}, \ldots, \varphi_{5}$ to obtain

$$
\begin{aligned}
\int_{\mathbb{R}^{N}}|\nabla \varphi|^{2} \leq & C\left(\left(\gamma^{2}+\delta^{N}+\gamma^{-4} \int_{B_{1}} \frac{\left(a_{\varepsilon}-|u|^{2}\right)^{2}}{\varepsilon^{2}}\right) E_{\varepsilon}(1)\right. \\
& \left.+\gamma^{-4}\left(\int_{B_{1}} \frac{\left(a_{\varepsilon}-|u|^{2}\right)^{2}}{\varepsilon^{2}}+\varepsilon^{\beta}\right)\right) .
\end{aligned}
$$


Step 3: Improved estimates for $\nabla\left(|u|^{2}\right)$ on $B_{\delta}$. The equation for $|u|^{2}$ reads

$$
\Delta\left(|u|^{2}\right)+2 \frac{\left(a_{\varepsilon}-|u|^{2}\right)|u|^{2}}{\varepsilon^{2}}=2|\nabla u|^{2}+2|\log \varepsilon|(i \mathbf{c} \cdot \nabla u, u) .
$$

Multiplying by $a_{\varepsilon}-|u|^{2}$ and integrating on $B_{r_{1}}$ we obtain

$$
\begin{aligned}
\int_{B_{r_{1}}}\left(\left.\left.|\nabla| u\right|^{2}\right|^{2}\right. & \left.+2 \frac{\left(a_{\varepsilon}-|u|^{2}\right)^{2}|u|^{2}}{\varepsilon^{2}}\right) \\
= & 2 \int_{B_{r_{1}}}\left(a_{\varepsilon}-|u|^{2}\right)|\nabla u|^{2}+\int_{\partial B_{r_{1}}}\left(a_{\varepsilon}-|u|^{2}\right) \frac{\partial|u|^{2}}{\partial n} \\
& +\int_{B_{r_{1}}} \nabla|u|^{2} \cdot \nabla a_{\varepsilon}+\int_{B_{r_{1}}} 2|\log \varepsilon|(i \mathbf{c} \cdot \nabla u, u)\left(a_{\varepsilon}-|u|^{2}\right) .
\end{aligned}
$$

From (A-31) we deduce

$$
\left|\int_{\partial B_{r_{1}}}\left(a_{\varepsilon}-|u|^{2}\right) \frac{\partial|u|^{2}}{\partial n}\right| \leq C \varepsilon\left(\int_{B_{1}} \frac{\left(a_{\varepsilon}-|u|^{2}\right)^{2}}{\varepsilon^{2}}\right)^{1 / 2}\left(\int_{B_{1}}|\nabla u|^{2}\right)^{1 / 2} .
$$

We also have

$$
\begin{aligned}
\left.\left|\int_{B_{r_{1}}}\left(a_{\varepsilon}-|u|^{2}\right)\right| \nabla u\right|^{2} \mid & \leq C \int_{V_{\gamma^{2}}} \gamma^{2}|\nabla u|^{2}+C \gamma^{-2} \int_{W_{\gamma^{2}}} \frac{\left(a_{\varepsilon}-|u|^{2}\right)^{2}}{\varepsilon^{2}} \\
& \leq C \gamma^{2} \int_{B_{1}}|\nabla u|^{2}+C \gamma^{-2} \int_{B_{1}} \frac{\left(a_{\varepsilon}-|u|^{2}\right)^{2}}{\varepsilon^{2}}
\end{aligned}
$$

On the other hand,

$$
\begin{aligned}
&\left|\int_{B_{r_{1}}} 2\right| \log \varepsilon \mid(i \mathbf{c} \cdot \nabla u, u)\left(a_{\varepsilon}-|u|^{2}\right) \mid \\
& \leq C \varepsilon|\log \varepsilon|\left(\int_{B_{1}} \frac{\left(a_{\varepsilon}-|u|^{2}\right)^{2}}{\varepsilon^{2}}\right)^{1 / 2}\left(\int_{B_{1}}|\nabla u|^{2}\right)^{1 / 2},
\end{aligned}
$$

and

$$
\left.\left.\left|\int_{B_{r_{1}}} \nabla\right| u\right|^{2} \cdot \nabla a_{\varepsilon}\left|\leq \frac{1}{2} \int_{B_{r_{1}}}\right| \nabla|u|^{2}\right|^{2}+2 \varepsilon^{4}|\log \varepsilon|^{4} \int_{B_{r_{1}}}|\nabla d|^{2} .
$$

Inserting (A-51 $-\mathrm{A}-54$ in $\mathrm{A}-50$ we finally obtain the estimate

$$
\left.\left.\int_{B_{r_{1}}}|\nabla| u\right|^{2}\right|^{2} \leq C\left(\gamma^{2} \int_{B_{1}}|\nabla u|^{2}+\gamma^{-2} \int_{B_{1}} \frac{\left(a_{\varepsilon}-|u|^{2}\right)^{2}}{\varepsilon^{2}}+\varepsilon^{\beta}\right) .
$$

Step 4: Proof of Lemma A.9 completed. Recall that

$$
4|u|^{2}|\nabla u|^{2}=4|u \times \nabla u|^{2}+\left.\left.|\nabla| u\right|^{2}\right|^{2},
$$


and thus

$$
\begin{aligned}
\left(3+a_{\varepsilon}\right)|\nabla u|^{2}= & 4|u \times \nabla u|^{2}+\left.\left.|\nabla| u\right|^{2}\right|^{2}+4\left(a_{\varepsilon}-|u|^{2}\right)|\nabla u|^{2} \\
\leq & 8\left(|\nabla \varphi|^{2}+|\nabla \xi|^{2}+\left(1-|u|^{2}\right)^{2}\left|\sum c_{i}(x) d x_{i}\right|^{2}|\log \varepsilon|^{2}\right) \\
& +\left.\left.|\nabla| u\right|^{2}\right|^{2}+4\left(a_{\varepsilon}-|u|^{2}\right)|\nabla u|^{2},
\end{aligned}
$$

by $(\mathrm{A}-38)$. Combining $\mathrm{A}-49, \mathrm{~A}-33, \mathrm{~A}-55, \mathrm{~A}-52$ and the easy estimate

$$
\int_{B_{\delta}}\left(1-|u|^{2}\right)^{2}\left|\sum c_{i}(x) d x_{i}\right|^{2}|\log \varepsilon|^{2} \leq C\left(\varepsilon^{2}|\log \varepsilon|^{2} \int_{B_{1}} \frac{\left(a_{\varepsilon}-|u|^{2}\right)^{2}}{\varepsilon^{2}}+\varepsilon^{\beta}\right),
$$

we finally obtain

$$
\begin{aligned}
E_{\varepsilon}(\delta) \leq & C\left(\left(\gamma^{2}+\delta^{N}+\gamma^{-4} \int_{B_{1}} \frac{\left(a_{\varepsilon}-|u|^{2}\right)^{2}}{\varepsilon^{2}}\right) E_{\varepsilon}(1)\right. \\
& \left.+\gamma^{-4}\left(\int_{B_{1}} \frac{\left(a_{\varepsilon}-|u|^{2}\right)^{2}}{\varepsilon^{2}}+\varepsilon^{\beta}\right)\right)
\end{aligned}
$$

which is the desired estimate. This ends the proof.

\section{Part C: Proof of Theorem 2 completed}

Remember that we are concerned with a solution $w$ of (124) with $\Lambda_{0} \leq 1$ on $B_{1}$ satisfying the estimate

$$
E_{\varepsilon}(w, 0,1) \leq \eta|\log \varepsilon| .
$$

Recall also that in Part A we have exhibited some $r_{0} \in\left(\varepsilon^{\alpha /(2 N-2)}, 1\right)$ such that

$$
\begin{aligned}
& \frac{1}{r_{0}^{N-2}} \int_{B_{r_{0}}} \frac{\left(a_{\varepsilon}-|w|^{2}\right)^{2}}{2 \varepsilon^{2}} \leq C\left(\eta|\log \delta|+\varepsilon^{\beta}\right), \\
& \tilde{E}_{\varepsilon}\left(r_{0}\right)-2^{N-2} \tilde{E}_{\varepsilon}\left(\delta r_{0}\right) \leq C\left(\eta|\log \delta|+\varepsilon^{\beta}\right),
\end{aligned}
$$

where $\delta$ is fixed but to be determined later. The function $u(x):=w\left(r_{0} x\right)$ defined on $B_{1}$ satisfies the equation

$$
\Delta u+\frac{1}{\tilde{\varepsilon}^{2}} u\left(1-|u|^{2}\right)=i \tilde{c} \cdot \nabla u|\log \tilde{\varepsilon}|+\tilde{d}|\log \tilde{\varepsilon}|^{2} u,
$$

where $\tilde{\varepsilon}:=\varepsilon / r_{0}$ and $\Lambda_{0}(\tilde{c}, \tilde{d}) \leq 1$. Since $r_{0} \geq \varepsilon^{\alpha /(2 N-2)}$, we have $\tilde{\varepsilon} \leq \varepsilon^{1 / 2}$. By scaling we also have the identities

$$
\begin{aligned}
& E_{\tilde{\varepsilon}}(u, 0,1)=\tilde{E}_{\varepsilon}\left(w, 0, r_{0}\right), \\
& E_{\tilde{\varepsilon}}(u, 0, \delta)=\frac{1}{r_{0}^{N-2}} E_{\varepsilon}\left(w, 0, \delta r_{0}\right)=\delta^{2-N} \tilde{E}_{\varepsilon}\left(w, 0, \delta r_{0}\right),
\end{aligned}
$$

and

$$
\int_{B_{1}} \frac{\left(a_{\tilde{\varepsilon}}-|u|^{2}\right)^{2}}{\tilde{\varepsilon}^{2}}=\frac{1}{r_{0}^{N-2}} \int_{B_{r_{0}}} \frac{\left(a_{\varepsilon}-|w|^{2}\right)^{2}}{\varepsilon^{2}}
$$


We now apply Lemma A.9 to $u$, and using the previous identities we find

$$
\begin{aligned}
\frac{1}{r_{0}^{N-2}} E_{\varepsilon}\left(\delta r_{0}\right) \leq & C\left(\left(\gamma^{2}+\delta^{N}+\gamma^{-4} \frac{1}{r_{0}^{N-2}} \int_{B_{r_{0}}} \frac{\left(a_{\varepsilon}-|w|^{2}\right)^{2}}{\varepsilon^{2}}\right) \tilde{E}_{\varepsilon}\left(r_{0}\right)\right. \\
& \left.+\gamma^{-4}\left(\frac{1}{r_{0}^{N-2}} \int_{B_{r_{0}}} \frac{\left(a_{\varepsilon}-|w|^{2}\right)^{2}}{\varepsilon^{2}}+\varepsilon^{\beta}\right)\right) .
\end{aligned}
$$

Using (A-57) and $\mathrm{A}-58$ ) we obtain

$$
\begin{aligned}
\tilde{E}_{\varepsilon}\left(r_{0}\right) \leq & 2^{N-2} \tilde{E}_{\varepsilon}\left(\delta r_{0}\right)+C\left(\eta|\log \delta|+\varepsilon^{\beta}\right) \\
\leq & C \delta^{N-2}\left(\gamma^{2}+\gamma^{-4}\left(\eta|\log \delta|+\varepsilon^{\beta}\right)\right) \tilde{E}_{\varepsilon}\left(r_{0}\right)+C \delta^{2} \tilde{E}_{\varepsilon}\left(r_{0}\right) \\
& +C \gamma^{-4}\left(\eta|\log \delta|+\varepsilon^{\beta}\right) .
\end{aligned}
$$

We now fix the values of $\delta$ and $\gamma$. First, choose $\delta$ small enough so that

$$
C \delta^{2} \leq 1 / 4
$$

Next, choose $\gamma$ small enough so that

$$
C \delta^{N-2} \gamma^{2} \leq 1 / 4
$$

There also exist $\varepsilon_{N}$ and $\eta_{N}$ such that if $\varepsilon<\varepsilon_{N}$ and $\eta \leq \eta_{N}$ then

$$
C \delta^{N-2} \gamma^{-4}\left(\eta|\log \delta|+\varepsilon^{\beta}\right) \leq 1 / 4 .
$$

Hence,

$$
\tilde{E}_{\varepsilon}\left(r_{0}\right) \leq C \gamma^{-4}\left(\eta|\log \delta|+\varepsilon^{\beta}\right) \quad \text { for } \varepsilon<\varepsilon_{N}, \eta<\eta_{N} .
$$

Using the monotonicity formula of Proposition 2 , we thus obtain

$$
\begin{aligned}
\frac{1}{\varepsilon^{N}} \int_{B_{\varepsilon}}\left(1-|u|^{2}\right)^{2} & \leq C\left(\frac{1}{\varepsilon^{N}} \int_{B_{\varepsilon}}\left(a_{\varepsilon}-|u|^{2}\right)^{2}+\Lambda_{0}^{2} \varepsilon^{\beta}\right) \leq C\left(\tilde{E}_{\varepsilon}(\varepsilon)+\Lambda_{0}^{2} \varepsilon^{\beta}\right) \\
& \leq C\left(\tilde{E}_{\varepsilon}\left(r_{0}\right)+\Lambda_{0}^{2} \varepsilon^{\beta}\right) \leq C \gamma^{-4}\left(\eta|\log \delta|+\Lambda_{0}^{2} \varepsilon^{\beta}\right) .
\end{aligned}
$$

The conclusion then follows from the next lemma taken from [10].

Lemma A.10. Let $w$ be a solution of (124) on $B_{1}$. Then

$$
1-|w(0)| \leq C\left(\frac{1}{\varepsilon^{N}} \int_{B_{\varepsilon}}\left(1-|w|^{2}\right)^{2}\right)^{1 /(N+2)} .
$$

Proof. Set $k=|w(0)|$ and assume that $k \leq 1$ (otherwise there is nothing to be proved). By $[\mathrm{A}-30]$ we have

$$
|w(x)-w(0)| \leq \frac{C}{\varepsilon}|x| \leq 1-\frac{k}{2},
$$

provided $|x| \leq \varepsilon(1-k) /(2 C) \equiv \lambda$. Therefore $|w(x)| \leq(1+k) / 2$ on $B_{\lambda}$. We distinguish two cases. 
Case 1: $\lambda<\varepsilon$. Then

$$
\int_{B_{\lambda}}\left(1-|w|^{2}\right)^{2} \leq \int_{B_{\varepsilon}}\left(1-|w|^{2}\right)^{2}
$$

On the other hand,

$$
\int_{B_{\lambda}}\left(1-|w|^{2}\right)^{2} \geq \int_{B_{\lambda}}(1-|w|)^{2} \geq\left(\frac{1-k}{2}\right)^{2}\left|B_{\lambda}\right|=C \varepsilon^{N}(1-k)^{N+2},
$$

by definition of $\lambda$. Consequently,

$$
(1-k)^{N+2} \leq \frac{C}{\varepsilon^{N}} \int_{B_{\varepsilon}}\left(1-|w|^{2}\right)^{2}
$$

and the conclusion follows.

Case 2: $\lambda \geq \varepsilon$. Then

$$
|w(x)| \leq \frac{1+k}{2} \quad \text { in } B_{\varepsilon},
$$

and

$$
\int_{B_{\varepsilon}}\left(1-|w|^{2}\right)^{2} \geq\left(\frac{1-k}{2}\right)^{2}\left|B_{\varepsilon}\right|
$$

Therefore

$$
(1-k)^{N+2} \leq(1-k)^{2} \leq \frac{C}{\varepsilon^{N}} \int_{B_{\varepsilon}}\left(1-|w|^{2}\right)^{2},
$$

and the lemma is proved.

Corollary A.1. Let $0<\sigma<1$, and let $\eta>0$ and $\varepsilon_{0}>0$ be given by Theorem 2. Let $x_{0} \in \Omega$ and $r>0$ be such that $B\left(x_{0}, 2 r\right) \subset \Omega$ and $4 \sqrt{\varepsilon}<r<4 /\left(1+\Lambda_{0}\right)$. Then for all $\varepsilon<\varepsilon_{0}$, if $w$ is a solution of (124) in $\Omega$ and

$$
\tilde{E}_{\varepsilon}\left(x_{0}, r\right) \leq 4^{2-N} \eta|\log \varepsilon|,
$$

then

$$
|1-| w(x)|| \leq \sigma \quad \text { for all } x \in B\left(x_{0}, 3 r / 4\right) .
$$

Proof. If $x \in B\left(x_{0}, 3 r / 4\right)$, then $B(x, r / 4) \subset B\left(x_{0}, r\right)$ so that

$$
\tilde{E}_{\varepsilon}(x, r / 4)=4^{N-2} \frac{1}{r^{N-2}} E_{\varepsilon}(x, r / 4) \leq 4^{N-2} \tilde{E}_{\varepsilon}\left(x_{0}, r\right) \leq \eta|\log \varepsilon|,
$$

and the conclusion follows by Theorem 2

Concerning the asymptotics of the potential part in the energy, namely

$$
\int_{\Omega} \frac{\left(a_{\varepsilon}(x)-|w|^{2}\right)^{2}}{\varepsilon^{2}}
$$

it is tempting to believe that it remains bounded as $\varepsilon \rightarrow 0$ (at least away from the boundary). We have no proof of that fact; however, the following proposition holds. 
Proposition A.1. Let $K \subset \Omega$ be a compact subset and $w$ a solution of (A.1) satisfying (24). Then

$$
\int_{K} \frac{\left(a_{\varepsilon}(x)-|w|^{2}\right)^{2}}{\varepsilon^{2}} \leq C r(\varepsilon)|\log \varepsilon|,
$$

where $r(\varepsilon) \rightarrow 0$ as $\varepsilon \rightarrow 0$ and $C$ depends only on $M_{0}$.

Proof. Let $\rho:=|w|$. If $w$ satisfies (124) then $\rho$ satisfies

$$
-\Delta \rho^{2}+2|\nabla w|^{2}=\frac{2}{\varepsilon^{2}} \rho^{2}\left(a_{\varepsilon}-\rho^{2}\right)-(w, i \mathbf{c} \cdot \nabla w)|\log \varepsilon| .
$$

Let $0<\sigma<1 / 2$. Define $A:=\{x \in K: \rho(x)>1-\sigma\}$ and $\bar{\rho}:=\max (\rho, 1-\sigma)$, so that $\rho=\bar{\rho}$ on $A$. Let also $\zeta \in \mathcal{D}(\Omega)$ be such that $0 \leq \zeta \leq 1$ on $\Omega, \zeta \equiv 1$ on $K$, and $|\nabla \zeta| \leq C$, where $C$ depends only on $K$. Multiplying equation $\mathrm{A}-63$ by $\zeta\left(\bar{\rho}^{2}-1\right)$ (which is compactly supported in $\Omega$ ) and integrating over $\Omega$ we obtain

$$
\begin{aligned}
\int_{\Omega} \nabla \rho^{2} \nabla \bar{\rho}^{2} \zeta+\int_{\Omega} \frac{2 \rho\left(1-\rho^{2}\right)\left(1-\bar{\rho}^{2}\right)}{\varepsilon^{2}} \zeta= & \int_{\Omega}\left(1-\bar{\rho}^{2}\right)|\nabla w|^{2}+\int_{\Omega} \nabla \rho^{2} \nabla \zeta\left(1-\bar{\rho}^{2}\right) \\
& +\int_{\Omega} 2 \rho|\log \varepsilon|^{2} d(x)\left(1-\bar{\rho}^{2}\right) \zeta \\
& +\int_{\Omega}(w, i \mathbf{c} \cdot \nabla w)\left(\bar{\rho}^{2}-1\right) \zeta|\log \varepsilon|
\end{aligned}
$$

It follows that

$$
\begin{aligned}
\int_{\Omega} \frac{2 \rho}{}\left(1-\rho^{2}\right)\left(1-\bar{\rho}^{2}\right) & \varepsilon^{2} \\
& \leq 2 \sigma \rho^{2} \nabla \bar{\rho}^{2} \\
& |\nabla w|^{2}+C \sigma \int_{\Omega}|\nabla \rho|\left|a_{\varepsilon}-\rho^{2}\right|+C \Lambda_{0} M_{0} \varepsilon|\log \varepsilon|^{2} \\
& \leq 2 \sigma \int_{\Omega}|\nabla w|^{2}+C \sigma\left[\int_{\Omega}|\nabla \rho|^{2}+\int_{\Omega} \frac{\left(a_{\varepsilon}-\rho^{2}\right)^{2}}{4 \varepsilon^{2}}\right]+C \Lambda_{0} M_{0} \varepsilon|\log \varepsilon|^{2} .
\end{aligned}
$$

Hence, since $\rho \geq 1 / 2$ and $\zeta=1$ on $A$, we obtain

$$
\int_{A} \frac{\left(a_{\varepsilon}-\rho^{2}\right)^{2}}{\varepsilon^{2}} \leq C \sigma E_{\varepsilon}(w)+C \Lambda_{0} M_{0} \varepsilon|\log \varepsilon|^{2} .
$$

Define also $B:=K \backslash A$. We claim that

$$
\int_{B} \frac{\left(a_{\varepsilon}-\rho^{2}\right)^{2}}{\varepsilon^{2}} \leq C,
$$

where $C$ depends only on $\sigma, M_{0}$ and $K$. This follows from Theorem 2, the monotonicity formula in Lemma 4 and the Besicovitch covering theorem, along the same lines as the proof of Proposition 1 in [10]. [Indeed, only the aforementioned ingredients are used and hence the proof there applies also to our equation.] In particular, we infer from A-65 that there exists $\varepsilon_{\sigma}>0$ such that

$$
\int_{B} \frac{\left(a_{\varepsilon}-\rho^{2}\right)^{2}}{\varepsilon^{2}} \leq \sigma|\log \varepsilon|
$$


for all $0<\varepsilon<\varepsilon_{\sigma}$, where $\varepsilon_{\sigma}$ depends only on $\sigma, M_{0}$ and $K$. Combining A-64 and A-66 we finally obtain

$$
\int_{K} \frac{\left(a_{\varepsilon}(x)-|w|^{2}\right)^{2}}{\varepsilon^{2}} \leq C \sigma|\log \varepsilon| \quad \text { for } 0<\varepsilon<\varepsilon_{\sigma} .
$$

Clearly we can assume that the mapping $t: \sigma \mapsto \varepsilon_{\sigma}$ is strictly increasing. The function $r:=t^{-1}$ fulfills the statement of the proposition, so that the proof is complete.

\section{Appendix B: Properties of the concentration set $\Sigma_{\mu}$}

Recall that

$$
\Sigma_{\mu}=\left\{x \in \Omega: \Theta_{*}\left(\mu_{*}, x\right)>0\right\} .
$$

The purpose of this section is to describe and prove the properties of $\Sigma_{\mu}$ stated in Theorem 3 . We first have

Lemma B.11. There exists $\eta_{0}>0$ such that if $x_{0} \in \Sigma_{\mu}$, then

$$
\Theta_{*}\left(\mu_{*}, x_{0}\right) \geq \eta_{0} .
$$

Proof. Let $\sigma>0$, to be determined later, and let $\eta>0$ and $\varepsilon_{0}>0$ be the corresponding constants provided by Theorem 2 Set

$$
\eta_{0}=4^{2-N} \eta
$$

Assume by contradiction that

$$
\Theta_{*}\left(x_{0}\right)<4^{2-N} \eta .
$$

Then for each $r_{0}>0$ there exists $0<r<r_{0}$ such that $B\left(x_{0}, 2 r\right) \subset \Omega$, and $\varepsilon_{1} \leq$ $\min \left(\varepsilon_{0}, r^{2} / 16\right)$ such that

$$
\tilde{E}_{\varepsilon}\left(x_{0}, r\right)<4^{2-N} \eta|\log \varepsilon| \quad \forall \varepsilon \leq \varepsilon_{1} .
$$

From Corollary A.1 we thus infer that

$$
|1-| w(x)|| \leq \sigma \quad \forall x \in B\left(x_{0}, 3 r / 4\right) .
$$

We write

$$
w(x)=\rho(x) \exp (i \varphi(x)) \quad \text { in } B\left(x_{0}, 3 r / 4\right) .
$$

The phase $\varphi$ satisfies the equation

$$
-\Delta \varphi=-\operatorname{div}\left(\left(1-\rho^{2}\right) \nabla \varphi\right)+\frac{1}{2}|\log \varepsilon| \mathbf{c} \cdot \nabla\left(\rho^{2}-1\right) \quad \text { in } B\left(x_{0}, 3 r / 4\right) .
$$

Let $\tilde{\varphi}$ be the harmonic function defined on $B\left(x_{0}, 3 r / 4\right)$ such that $\tilde{\varphi}=\varphi$ on the boundary of $B\left(x_{0}, 3 r / 4\right)$. In particular, we have

$$
\int_{B\left(x_{0}, 3 r / 4\right)}|\nabla \tilde{\varphi}|^{2} \leq \int_{B\left(x_{0}, 3 r / 4\right)}|\nabla \varphi|^{2}
$$


and for all $\delta>0$,

$$
\int_{B\left(x_{0}, \delta 3 r / 4\right)}|\nabla \tilde{\varphi}|^{2} \leq C \delta^{N} \int_{B\left(x_{0}, 3 r / 4\right)}|\nabla \tilde{\varphi}|^{2} \leq C \delta^{N} \int_{B\left(x_{0}, 3 r / 4\right)}|\nabla \varphi|^{2} .
$$

Multiplying equation $(\mathrm{B}-3)$ by $\varphi-\tilde{\varphi}$ and integrating over $B\left(x_{0}, 3 r / 4\right)$ we obtain, as in the proof of Theorem 2

$$
\int_{B\left(x_{0}, \delta 3 r / 4\right)}|\nabla(\varphi-\tilde{\varphi})|^{2} \leq C\left(\sigma+\Lambda_{0} \varepsilon|\log \varepsilon|\right) E_{\varepsilon}\left(x_{0}, 3 r / 4\right) .
$$

Combining $(\mathrm{B}-4)$ and $(\bar{B}-5)$ we finally obtain

$$
\int_{B\left(x_{0}, \delta 3 r / 4\right)}|\nabla \varphi|^{2} \leq C\left(\delta^{N}+\sigma+\Lambda_{0} \varepsilon|\log \varepsilon|\right) E_{\varepsilon}\left(x_{0}, 3 r / 4\right) .
$$

Concerning the modulus, let $\xi_{r} \in \mathcal{D}\left(B\left(x_{0}, 3 r / 4\right),[0,1]\right)$ be such that $\xi \equiv 1$ on $B\left(x_{0}, 3 r / 8\right)$ and $\left|\nabla \xi_{r}\right| \leq C / r$. Multiplying the equation

$$
-\Delta \rho+\rho|\nabla \varphi|^{2}=\frac{1}{\varepsilon^{2}} \rho\left(a_{\varepsilon}-\rho^{2}\right)+|\log \varepsilon| \rho \mathbf{c} \cdot \nabla \varphi
$$

by $\xi_{r}(1-\rho)$ and integrating over $B\left(x_{0}, 3 r / 4\right)$ we obtain

$$
\begin{aligned}
\int_{B\left(x_{0}, 3 r / 8\right)}|\nabla \rho|^{2}+\frac{\left(a_{\varepsilon}-\rho^{2}\right)^{2}}{\varepsilon^{2}} \leq & C \sigma \int_{B\left(x_{0}, 3 r / 4\right)}|\nabla w|^{2} \\
& +C \frac{\varepsilon}{r} E_{\varepsilon}\left(x_{0}, 3 r / 4\right)+C \Lambda_{0} \varepsilon|\log \varepsilon|^{2} .
\end{aligned}
$$

Hence, since $r \geq 4 \sqrt{\varepsilon}$, from (B-6) and (B-7) we have

$$
\tilde{E}_{\varepsilon}\left(x_{0}, \delta 3 r / 4\right) \leq C\left(\delta^{2}+\delta^{2-N}\left(\sigma+\Lambda_{0} \varepsilon|\log \varepsilon|+\varepsilon^{1 / 2}\right) \tilde{E}_{\varepsilon}\left(x_{0}, r\right)+C \frac{\Lambda_{0} \varepsilon|\log \varepsilon|^{2}}{(\delta r)^{N-2}} .\right.
$$

Now choose $\delta$ such that $C \delta^{2}<1 / 4$ and then $\sigma$ such that $C \delta^{2-N} \sigma<1 / 4$. Letting $\varepsilon$ tend to zero in the previous inequality keeping $r$ fixed yields

$$
\frac{\mu_{*}\left(B\left(x_{0}, \delta 3 r / 4\right)\right.}{(\delta 3 r / 4)^{N-2}} \leq \frac{1}{2} \frac{\mu_{*}\left(B\left(x_{0}, r\right)\right)}{r^{N-2}} .
$$

Since $r<r_{0}$, and $r_{0}$ was arbitrarily small, we infer taking a sequence $r_{0} \rightarrow 0$ that

$$
\Theta_{*}\left(x_{0}\right) \leq \frac{1}{2} \Theta_{*}\left(x_{0}\right), \quad \text { i.e. } \quad \Theta_{*}\left(x_{0}\right)=0 .
$$

This contradicts the definition of $\Sigma_{\mu}$ and the proof is complete.

Lemma B.12. $\Sigma_{\mu}$ is closed in $\Omega$.

Proof. This follows directly from the upper semicontinuity of $\Theta_{*}$, the lower density.

Lemma B.13 (Uniform convergence away from $\Sigma_{\mu}$ ). Let $K \subset \Omega \backslash \Sigma_{\mu}$ be any compact subset. For any $\sigma>0$, there exists $\tilde{\varepsilon}>0$ depending only on $K$ and $\sigma$ such that if $0<\varepsilon<\tilde{\varepsilon}$, then

$$
|1-| w|| \leq \sigma \quad \text { on } K \text {. }
$$


Proof. Let $\sigma>0$ and let the corresponding $\eta>0$ and $\varepsilon_{0}>0$ be given by Theorem 2 For each $x \in K$, we deduce from Lemma B.11 that there exist $r(x)>0$ and $\varepsilon(x)>0$ such that

$$
\tilde{E}_{\varepsilon}(x, r(x)) \leq 4^{2-N} \eta|\log \varepsilon| \quad \forall \varepsilon \leq \varepsilon(x) .
$$

Let $x_{1}, \ldots, x_{k}$ be such that

$$
K \subset \bigcup_{i=1}^{k} B\left(x_{i}, r\left(x_{i}\right) / 2\right)
$$

and let $\tilde{\varepsilon}:=\min \left(\varepsilon_{0}, \varepsilon\left(x_{1}\right), \ldots, \varepsilon\left(x_{k}\right)\right)$. From Corollary A.1, it follows that for $\varepsilon \leq \tilde{\varepsilon}$,

$$
|1-| w|| \leq \sigma \quad \text { on } B\left(x_{i}, r\left(x_{i}\right) / 2\right) \quad \forall i=1, \ldots, k .
$$

This proves the lemma.

Lemma B.14 (Structure of $\mu_{*}$ ). We have

$$
\mu_{*}=g(x) \mathcal{H}^{N}+h(x) \mathcal{H}^{N-2}\left\llcorner\Sigma_{\mu},\right.
$$

where $g$ and $h$ are locally bounded on $\Omega$ and $h$ satisfies

$$
\eta_{0} \leq \Theta_{*}(x)<h(x) \leq \Theta^{*}(x) \equiv \limsup _{r \rightarrow 0} \frac{\mu_{*}(B(x, r))}{r^{N-2}} \leq c(x) M_{0} .
$$

Proof. Since $\Sigma_{\mu}$ is closed in $\Omega$ and hence measurable, we have

$$
\mu_{*}=\mu_{*}\left\llcorner\Sigma_{\mu}+\mu_{*}\left\llcorner\left(\Omega \backslash \Sigma_{\mu}\right) .\right.\right.
$$

As in [10, Theorem VIII.1], we infer from Corollary A.1 that $\mathcal{H}^{N-2}\left(\Sigma_{\mu}\right) \leq C M_{0}$. It also follows from the monotonicity formula of Proposition 2 that for all $x \in \Omega$,

$$
\Theta^{*}(x):=\limsup _{r \rightarrow 0} \frac{\mu_{*}(B(x, r))}{r^{N-2}} \leq C M_{0} .
$$

Using the Radon-Nikodym theorem, we thus obtain

$$
\mu_{*}\left\llcorner\Sigma_{\mu}=h(x) \cdot \mathcal{H}^{N-2}\left\llcorner\Sigma_{\mu}\right.\right.
$$

for some $\Theta_{*} \leq h \leq \Theta^{*}$. We will prove that in fact $\Theta_{*}=\Theta^{*}$.

Now, let $x_{0} \in \Omega \backslash \Sigma_{\mu}$ and $r>0$ be such that $\overline{B\left(x_{0}, 2 r\right)} \subset \Omega \backslash \Sigma_{\mu}$. By Lemma B.13.

$$
\sigma:=\|1-|w|\|_{L^{\infty}\left(\overline{B\left(x_{0}, 2 r\right)}\right)}=o(1) \quad \text { as } \varepsilon \rightarrow 0 .
$$

The same computation as in Lemma B.11 (see (B-8) shows that for each $0<\delta<1 / 2$,

$$
E_{\varepsilon}\left(x_{0}, \delta 3 r / 4\right) \leq C\left(\delta^{N}+\sigma+\Lambda_{0} \varepsilon|\log \varepsilon|+\varepsilon^{1 / 2}\right) E_{\varepsilon}\left(x_{0}, r\right)+C \Lambda_{0} \varepsilon|\log \varepsilon|^{2},
$$

but now we know that $\sigma=\sigma(\varepsilon)=o(1)$. Hence, dividing both sides by $|\log \varepsilon|$ and sending $\varepsilon$ to zero we obtain

$$
\mu_{*}\left(B\left(x_{0}, \delta 3 r / 4\right)\right) \leq C \delta^{N} \mu_{*}\left(B\left(x_{0}, r\right)\right) .
$$

This implies that $\mu_{*}\left\llcorner\left(\Omega \backslash \Sigma_{\mu}\right)\right.$ is absolutely continuous with respect to the Lebesgue measure, and using the Radon-Nikodym theorem once more we finally deduce that

$$
\mu_{*}=g(x) \cdot \mathcal{H}^{N}+h(x) \cdot \mathcal{H}^{N-2}\left\llcorner\Sigma_{\mu}\right.
$$

for some locally bounded function $g$. 
Lemma B.15. We have

$$
g(x)=\left|\nabla h_{*}(x)\right|^{2} \quad \text { a.e. in } \Omega,
$$

where $h_{*}$ is some harmonic function.

Proof. The argument is similar to the one carried out in [12] for Theorem A(iv). Since the proof is rather lengthy we briefly sketch the main steps.

First, one has to prove that if $\left|w_{\varepsilon}\right| \geq 1-\sigma_{0}$ on some ball $B\left(x_{0}, R\right)$ (where $\sigma_{0}$ is some suitable constant), then

$$
\left|\nabla w_{\varepsilon}\right|^{2} \simeq\left|\nabla \phi_{\varepsilon}\right|^{2} \quad \text { on } B\left(x_{0}, 3 R / 4\right),
$$

where $\phi_{\varepsilon}$ is harmonic and satisfies

$$
\left|\nabla \phi_{\varepsilon}\right|^{2} \leq C \sqrt{M_{0}|\log \varepsilon|}
$$

Then

$$
\frac{\phi_{\varepsilon}}{\sqrt{|\log \varepsilon|}} \rightarrow h_{*},
$$

which is thus harmonic on $B\left(x_{0}, 3 R / 4\right)$.

A second important step is to prove that $h_{*}$ is globally well defined and harmonic on $\Omega$. Here the argument is the same as in [12].

Proof of the curvature equation and the rectifiability of $\Sigma_{\mu}$. Let $\mathbf{X} \in \mathcal{D}\left(\Omega, \mathbb{R}^{N}\right)$ be a smooth vector field and

$$
e_{\varepsilon}(w):=\frac{1}{2}|\nabla w|^{2}+\frac{1}{4 \varepsilon^{2}}\left(a_{\varepsilon}-|w|^{2}\right)^{2} .
$$

We have

$$
\begin{aligned}
\int_{\Omega} e_{\varepsilon}(w) & \operatorname{div} \mathbf{X}=-\int_{\Omega} \nabla e_{\varepsilon}(w) \cdot \mathbf{X} \\
=- & \int_{\Omega}\left(\frac{1}{2} \nabla\left(|\nabla w|^{2}\right)+\frac{1}{2 \varepsilon^{2}}\left(a_{\varepsilon}-|w|^{2}\right)\left(-2 w \nabla w+\nabla a_{\varepsilon}\right)\right) \cdot \mathbf{X},
\end{aligned}
$$

and

$$
\begin{aligned}
\int_{\Omega} \sum_{i, j} \frac{\partial w}{\partial x_{i}} \frac{\partial w}{\partial x_{j}} \frac{\partial X^{i}}{\partial x_{j}} & =-\int_{\Omega} \sum_{i, j}\left(\frac{\partial^{2} w}{\partial x_{i} \partial x_{j}} \frac{\partial w}{\partial x_{j}}+\frac{\partial w}{\partial x_{i}} \frac{\partial^{2} w}{\partial x_{j}^{2}}\right) X^{i} \\
& =-\int_{\Omega} \nabla w \cdot \mathbf{X} \Delta w-\int_{\Omega} \sum_{i, j} \frac{\partial}{\partial x_{i}}\left|\frac{\partial w}{\partial x_{j}}\right|^{2} X^{i} \\
& =-\int_{\Omega} \nabla w \cdot \mathbf{X} \Delta w-\int_{\Omega} \frac{1}{2} \nabla\left(|\nabla w|^{2}\right) \cdot \mathbf{X} .
\end{aligned}
$$


Since $w$ is a solution of (124), we deduce from (B-13) and (B-14) that

$$
\begin{aligned}
\frac{1}{|\log \varepsilon|} \int_{\Omega}\left(e_{\varepsilon}(w) \delta_{i j}-\frac{\partial w}{\partial x_{i}} \frac{\partial w}{\partial x_{j}}\right) \frac{\partial X^{i}}{\partial x_{j}} \\
\quad=\frac{1}{|\log \varepsilon|} \int_{\Omega}(\nabla w \cdot \mathbf{X})\left(\Delta w+\frac{1}{\varepsilon^{2}} w\left(a_{\varepsilon}-|w|^{2}\right)\right)+\frac{1}{2}\left(a_{\varepsilon}-|w|^{2}\right)|\log \varepsilon|^{2} \nabla d \cdot \mathbf{X} \\
\quad=\int_{\Omega}(\nabla w \cdot \mathbf{X}, i \mathbf{c} \cdot \nabla w)+\int_{\Omega} \frac{1}{2}\left(a_{\varepsilon}-|w|^{2}\right)|\log \varepsilon| \nabla d \cdot \mathbf{X} \\
=-\int_{\Omega}\langle *(\mathbf{c} \wedge * J w), \mathbf{X}\rangle+\int_{\Omega} \frac{1}{2}\left(a_{\varepsilon}-|w|^{2}\right)|\log \varepsilon| \nabla d \cdot \mathbf{X}
\end{aligned}
$$

Set

$$
\alpha_{\varepsilon}^{i j}:=\frac{1}{|\log \varepsilon|}\left(e_{\varepsilon}(w) \delta_{i j}-\frac{\partial w}{\partial x_{i}} \frac{\partial w}{\partial x_{j}}\right)
$$

Notice that $\alpha_{\varepsilon}^{i j}$ is a symmetric matrix with trace larger than $(N-2) \mu_{\varepsilon}$, and a little linear algebra shows that its eigenvalues are less than or equal to $\mu_{\varepsilon}$. Moreover,

$$
\left|\alpha_{\varepsilon}^{i j}\right| \leq N \mu_{\varepsilon}
$$

Going if necessary to a subsequence, we may thus assume that

$$
\alpha_{\varepsilon}^{i j} \rightarrow \alpha_{*}^{i j} \quad \text { in the sense of measures. }
$$

In view of $\mathrm{B}-16$ we have $\left|\alpha_{*}^{i j}\right| \leq N \mu_{*}$, therefore we may write

$$
\alpha_{*}^{i j}(x)=A^{i j}(x) \mu_{*} \quad \text { for } \mu_{*} \text {-a.e. } x \in \Omega,
$$

where the matrix $A^{i j}(x)$ is symmetric, with trace equal to $N-2$ and eigenvalues less than or equal to one [the fact that the trace is equal to $N-2$ and not just less than $N-2$ follows from Proposition A.1]. From B-16, we also have

$$
A^{i j} \geq-N \delta^{i j} \quad \text { for } \mu_{*} \text {-a.e. } x \in \Omega \text {. }
$$

Notice that

$$
\left|\int_{\Omega} \frac{1}{2}\left(a_{\varepsilon}-|w|^{2}\right)\right| \log \varepsilon|\nabla d \cdot \mathbf{X}| \leq C \Lambda_{0} \varepsilon|\log \varepsilon| \rightarrow 0 \quad \text { as } \varepsilon \rightarrow 0,
$$

so that passing to the limit in $B-15$ we obtain

$$
\begin{aligned}
\int_{\Omega} A^{i j}(x) \frac{\partial X^{i}}{\partial x_{j}} d \mu_{*}(x) & =-\int_{\Omega}\left\langle *\left(\mathbf{c}(x) \wedge * d J_{*}(x)\right), \mathbf{X}\right\rangle \\
& =-\int_{\Omega}\left\langle *\left(\mathbf{c} \wedge * \frac{d J_{*}}{d \mu_{*}}\right), \mathbf{X}\right\rangle d \mu_{*}(x) .
\end{aligned}
$$


We decompose the r.h.s. of $\mathrm{B}-18$ as

$$
\begin{aligned}
\int_{\Omega} A^{i j}(x) \frac{\partial X^{i}}{\partial x_{j}} d \mu_{*}(x)= & \int_{\Omega} A^{i j}(x) \frac{\partial X^{i}}{\partial x_{j}} d \mu_{*}(x)\left\llcorner\Sigma_{\mu}\right. \\
& +\int_{\Omega}\left(\frac{\left|\nabla h_{*}\right|^{2}}{2} \delta_{i j}-\frac{\partial h_{*}}{\partial x_{i}} \frac{\partial h_{*}}{\partial x_{j}}\right) \frac{\partial X^{i}}{\partial x_{j}} d x .
\end{aligned}
$$

Since $h_{*}$ is harmonic, the last term in $\mathrm{B}-19$ vanishes. Hence, the support of $J_{*}$ being included in $\Sigma_{\mu}$, using $(\bar{B}-12]$ we obtain

$$
\int_{\Omega} A^{i j}(x) \frac{\partial X^{i}}{\partial x_{j}} d \mu_{*}(x)\left\llcorner\Sigma_{\mu}=-\int_{\Omega}\left\langle *\left(\mathbf{c} \wedge * \frac{d J_{*}}{d \mu_{*}}\right), \mathbf{X}\right\rangle d \mu_{*}(x)\left\llcorner\Sigma_{\mu} .\right.\right.
$$

Since $\mathbf{X}$ was arbitrary, the previous equality means in particular that the generalized $(N-2)$-varifold (see [5])

$$
\tilde{V}:=\delta_{A^{i j}(x)} \mu_{*}\left\llcorner\Sigma_{\mu}(x)\right.
$$

has a first variation. From Step 1 and [5, Theorem 3.8(c)] we thus infer that $\tilde{V}$ is indeed a real rectifiable $(N-2)$-varifold. In particular, the geometrical support $\Sigma_{\mu}$ of $\mu_{*}\left\llcorner\Sigma_{\mu}\right.$ is rectifiable. From the rectifiability of $\Sigma_{\mu}$, we deduce that

$$
\Theta_{*}(x)=\Theta^{*}(x) \quad \text { for } \mu_{*} \text {-a.e. } x \text { in } \Sigma_{\mu},
$$

so that

$$
\mu_{*}=g(x) \cdot \mathcal{H}^{N}+\Theta_{*}(x) \cdot \mathcal{H}^{N-2}\left\llcorner\Sigma_{\mu},\right.
$$

and

$$
V\left(\Sigma_{\mu}, \Theta_{*}\right)=\tilde{V}
$$

Equation $(\mathrm{B}-20)$ then precisely states that $V\left(\Sigma_{\mu}, \Theta_{*}\right)$ satisfies the mean curvature equation

$$
\mathbf{H}(x)=*\left(\mathbf{c}(x) \wedge * \frac{d J_{*}}{d \mu_{*}}\right) \quad \text { for } \mu_{*} \text {-a.e. } x \text { in } \Sigma_{\mu} .
$$

The proof of Theorem 3 is now complete.

\section{Appendix C: Compactness}

If some additional conditions are imposed on the boundary data, we may obtain compactness properties for $w_{\varepsilon}$. In this part, we will assume

$$
\int_{\partial \Omega} e_{\varepsilon}(w) \leq M_{0}, \quad\|w\|_{H^{1 / 2}(\partial \Omega)} \leq M_{0} .
$$

[There are however many variants of condition (C-1), see [7, 11].]

Proposition C.2. Let $1 \leq p<N /(N-1)$. There exists a constant $C>0$ depending on $p, M_{0}, \Lambda_{0}$ and $\Omega$ but independent of $\varepsilon$ such that if $w$ is a solution of (124) satisfying (24) and (C-1) then

$$
\int_{\Omega}|\nabla w|^{p} \leq C .
$$


Proof. We follow the lines of [7, 11]. Let $\rho:=|w|$. From the identity

$$
\rho^{2}|\nabla w|^{2}=\rho^{2}|\nabla \rho|^{2}+|w \times \nabla w|^{2},
$$

and the inequality $|\nabla w| \geq|\nabla \rho|$, we deduce that

$$
\begin{aligned}
|\nabla w|^{2} & =|\nabla \rho|^{2}+|w \times \nabla w|^{2}+\left(1-|w|^{2}\right)\left(|\nabla w|^{2}-|\nabla \rho|^{2}\right) \\
& \leq|\nabla \rho|^{2}+|w \times \nabla w|^{2}+\left.\left.\left|a_{\varepsilon}-\right| w\right|^{2}|| \nabla w\right|^{2}+\Lambda_{0} \varepsilon^{2}|\log \varepsilon||\nabla w|^{2} \\
& \leq|\nabla \rho|^{2}+|w \times \nabla w|^{2}+\left(\sqrt{2} \varepsilon+\Lambda_{0} \varepsilon^{2}|\log \varepsilon|\right) e_{\varepsilon}(w) .
\end{aligned}
$$

Hence, since 24] is satisfied,

$$
\int_{\Omega}|\nabla w|^{p} \leq C\left[\int_{\Omega}|\nabla \rho|^{p}+\int_{\Omega}|w \times \nabla w|^{p}+1\right],
$$

where $C$ depends only on $p, \Lambda_{0}, M_{0}$ and $\Omega$.

Step 1: Estimates for the modulus. Notice that $\rho$ satisfies the equation

$$
-\Delta \rho^{2}+2|\nabla w|^{2}=\frac{2}{\varepsilon^{2}} \rho^{2}\left(a_{\varepsilon}-\rho^{2}\right)-(w, i \mathbf{c} \cdot \nabla w)|\log \varepsilon| .
$$

Let us introduce the set

$$
A=\left\{x \in \Omega: \rho(x)>1-\varepsilon^{1 / 2}\right\}
$$

and the function

$$
\bar{\rho}=\max \left\{\rho, 1-\varepsilon^{1 / 2}\right\},
$$

so that $\bar{\rho}=\rho$ on $A$ and $0 \leq 1-\bar{\rho} \leq \varepsilon^{1 / 2}$ in $\Omega$.

Next let $\zeta_{\varepsilon}$ be a function in $\mathcal{D}(\Omega)$ such that $0 \leq \zeta_{\varepsilon} \leq 1$ on $\Omega, \zeta_{\varepsilon} \equiv 1$ on $\Omega_{\varepsilon} \equiv$ $\left\{x \in \Omega: \operatorname{dist}(x, \partial \Omega) \geq \varepsilon^{1 / 2}\right\}$, and $\left|\nabla \zeta_{\varepsilon}\right| \leq C \varepsilon^{-1 / 2}$, where $C$ depends only on $\Omega$. By multiplying equation $\mathrm{C}-4$ by $\zeta_{\varepsilon}\left(\bar{\rho}^{2}-1\right)$ (which is compactly supported in $\Omega$ ), and integrating over $\Omega$ we obtain

$$
\begin{aligned}
\int_{\Omega} \nabla \rho^{2} \nabla & \bar{\rho}^{2} \zeta_{\varepsilon}+\int_{\Omega} \frac{2 \rho\left(1-\rho^{2}\right)\left(1-\bar{\rho}^{2}\right)}{\varepsilon^{2}} \zeta_{\varepsilon} \\
= & \int_{\Omega}\left(1-\bar{\rho}^{2}\right)|\nabla w|^{2}+\int_{\Omega} \nabla \rho^{2} \nabla \zeta_{\varepsilon}\left(1-\bar{\rho}^{2}\right) \\
& \quad+\int_{\Omega} 2 \rho|\log \varepsilon|^{2} d(x)\left(1-\bar{\rho}^{2}\right) \zeta_{\varepsilon}+\int_{\Omega}(w, i \mathbf{c} \cdot \nabla w)\left(\bar{\rho}^{2}-1\right) \zeta_{\varepsilon}|\log \varepsilon|
\end{aligned}
$$

It follows that on the set $A_{\varepsilon}=\Omega_{\varepsilon} \cap A$ we have

$$
\begin{aligned}
\int_{A_{\varepsilon}}\left|\nabla \rho^{2}\right|^{2} & =\int_{A_{\varepsilon}} \nabla \rho^{2} \nabla \bar{\rho}^{2} \\
& \leq 2 \varepsilon^{1 / 2} \int_{\Omega}|\nabla w|^{2}+\frac{C}{\varepsilon^{1 / 2}} \int_{\Omega}|\nabla \rho|\left|a_{\varepsilon}-\rho^{2}\right|+C \Lambda_{0} M_{0} \varepsilon|\log \varepsilon|^{2} \\
& \leq 2 \varepsilon^{1 / 2} \int_{\Omega}|\nabla w|^{2}+C \varepsilon^{1 / 2}\left[\int_{\Omega}|\nabla \rho|^{2}+\int_{\Omega} \frac{\left(a_{\varepsilon}-\rho^{2}\right)^{2}}{4 \varepsilon^{2}}\right]+C \Lambda_{0} M_{0} \varepsilon|\log \varepsilon|^{2} .
\end{aligned}
$$


Hence, since $\rho \geq 1-\varepsilon^{1 / 2}$ on $A_{\varepsilon}$, we have, for $\varepsilon \leq 1 / 4$,

$$
\int_{A_{\varepsilon}}|\nabla \rho|^{2} \leq 4 \int_{A_{\varepsilon}}\left|\nabla \rho^{2}\right|^{2} \leq C \varepsilon^{1 / 2} E_{\varepsilon}(w)+C \Lambda_{0} M_{0} \varepsilon|\log \varepsilon|^{2} \leq C .
$$

Set $W_{\varepsilon}=\Omega \backslash \Omega_{\varepsilon}, B=\Omega \backslash A$, so that

$$
\Omega=B \cup A_{\varepsilon} \cup W_{\varepsilon} .
$$

From 24 we deduce $\int_{B}\left(1-\rho^{2}\right)^{2} \leq 4 M_{0} \varepsilon^{2}|\log \varepsilon|$ and hence, since $1-\rho \geq \varepsilon^{1 / 2}$ on $B$, it follows that $|B| \leq 4 M_{0} \varepsilon|\log \varepsilon|$. Thus

$$
\int_{B}|\nabla \rho|^{p} \leq\left(\int_{\Omega}|\nabla \rho|^{2}\right)^{p / 2}|B|^{1-p / 2} \leq C|\log \varepsilon|^{p / 2}(\varepsilon|\log \varepsilon|)^{1-p / 2},
$$

i.e.

$$
\int_{B}|\nabla \rho|^{p} \leq C \varepsilon^{1-p / 2}|\log \varepsilon| .
$$

Finally, we turn to $W_{\varepsilon}$. Clearly, by construction $\left|W_{\varepsilon}\right| \leq C \varepsilon^{1 / 2}$. Hence

$$
\int_{W_{\varepsilon}}|\nabla \rho|^{p} \leq\left(\int_{\Omega}|\nabla \rho|^{2}\right)^{p / 2}\left|W_{\varepsilon}\right|^{1-p / 2} \leq C \varepsilon^{1 / 2-p / 4}|\log \varepsilon|^{p / 2} .
$$

Combining $(\overline{\mathrm{C}-5}-\overline{(\mathrm{C}-7)}$ we get the estimate for the modulus:

$$
\int_{\Omega}|\nabla \rho|^{p} \leq C
$$

where $C$ does not depend on $\varepsilon$.

Step 2: Estimates for the pre-Jacobian. Consider the Hodge-de Rham decomposition of $w \times \nabla w$ :

$$
w \times \nabla w=d \varphi+d^{*} \psi,
$$

where the function $\varphi$ satisfies $\varphi=0$ on $\partial \Omega$ and the 2-form $\psi$ satisfies $d \psi=0$ on $\Omega$ and $\psi_{\top}=0$ on $\partial \Omega$. Applying respectively the operators $d^{*}$ and $d$ to $[\mathrm{C}-9]$ we obtain the equation for $\varphi$ (resp. $\psi$ ):

$$
\begin{cases}\Delta \varphi=\mathbf{c} \cdot \nabla\left(|w|^{2}-1\right)|\log \varepsilon| & \text { in } \Omega, \\ \varphi=0 & \text { on } \partial \Omega\end{cases}
$$

and

$$
\begin{cases}\Delta \psi=2 J w & \text { in } \Omega, \\ \psi_{\top}=0, \quad\left(d^{*} \psi\right)_{\top}=(w \times d w)_{\top} & \text { on } \partial \Omega .\end{cases}
$$

From [24], [C-1], [C-11] and Proposition III.1 in [11] we infer that

$$
\int_{\Omega}|\nabla \psi|^{p} \leq C
$$


Indeed, the estimate $(\mathrm{C}-12)$ is valid even without assuming that $w$ is a solution of (124) (see [11]). Notice, however, that the constant $C$ may depend on $\Omega$; in the proof of Theorem 4 we will see how to use the extra information that $w$ satisfies 124 to obtain estimates independent of the domain.

Concerning $\varphi$, multiplying equation $(\mathrm{C}-10)$ by $\varphi$ and integrating over $\Omega$ we get

$$
\begin{aligned}
\int_{\Omega}|\nabla \varphi|^{2} & =|\log \varepsilon| \int_{\Omega} \operatorname{div}\left(\left(|w|^{2}-1\right) \mathbf{c}\right) \varphi \\
& =|\log \varepsilon| \int_{\Omega}\left(|w|^{2}-1\right) \mathbf{c} \cdot \nabla \varphi \\
& \leq C \Lambda_{0} \varepsilon|\log \varepsilon|\left(\int_{\Omega} \frac{\left(1-|w|^{2}\right)^{2}}{4 \varepsilon^{2}}\right)^{1 / 2}\left(\int_{\Omega}|\nabla \varphi|^{2}\right)^{1 / 2} \\
& \leq C \Lambda_{0}\left(M_{0}+1\right) \varepsilon|\log \varepsilon|^{3 / 2}\left(\int_{\Omega}|\nabla \varphi|^{2}\right)^{1 / 2}
\end{aligned}
$$

so that

$$
\int_{\Omega}|\nabla \varphi|^{p} \leq\left(\int_{\Omega}|\nabla \varphi|^{2}\right)^{p / 2}|\Omega|^{1-p / 2} \leq C,
$$

where $C$ does not depend on $\varepsilon$. Combining $(\mathrm{C}-8),(\mathrm{C}-12)$ and $(\mathrm{C}-14)$ we get the desired conclusion from (C-3).

Proof of Theorem 4 Recall that $w_{\varepsilon}$ is a solution of (11) on $\Pi_{n}$ such that 24 and (31) are satisfied. For simplicity, we omit the subscripts $\varepsilon$ below, i.e. we set $w \equiv w_{\varepsilon}$.

Step 1: Extracting the "bad" balls. From Theorem 2, we infer that there exists $\eta>0$ and $R_{0}>0$ such that for each $x \in S_{\varepsilon}$,

$$
E_{\varepsilon}\left(x, R_{0}\right) \geq \eta|\log \varepsilon| .
$$

It follows from Vitali's covering theorem that there exist an at most countable family $\left(y_{i, \varepsilon}\right)_{i \in I}$ of points in $S_{\varepsilon}$ such that

$$
S_{\varepsilon} \subset \bigcup_{i \in I} B\left(y_{i, \varepsilon}, 5 R_{0}\right)
$$

and

$$
B\left(y_{i, \varepsilon}, R_{0}\right) \cap B\left(y_{j, \varepsilon}, R_{0}\right)=\emptyset \quad \text { if } i \neq j .
$$

We deduce from (24, $\mathrm{C}-15$ and the previous equality that

$$
\sharp I \leq l:=M_{0} / \eta \text {. }
$$

We claim that there exists a constant $10 \leq \kappa \leq C(\sharp I)$ (where $C(\sharp I)$ depends only on $\sharp I)$ and $q$ points $x_{1, \varepsilon}, \ldots, x_{q, \varepsilon} \in \Pi_{n}(q \leq l)$ such that, with $R:=\kappa R_{0}$,

$$
S_{\varepsilon} \subset \bigcup_{i=1}^{q} B\left(x_{i, \varepsilon}, R\right) \text { and } \operatorname{dist}\left(x_{i, \varepsilon}, x_{j, \varepsilon}\right) \geq 10 R \quad \text { if } i \neq j .
$$

Indeed, set $R_{1}:=10 R_{0}$. If $\operatorname{dist}\left(y_{i, \varepsilon}, y_{j, \varepsilon}\right) \geq 10 R_{1}$ there is nothing to prove. If not, consider the equivalence relation

$$
y_{i, \varepsilon} \sim y_{j, \varepsilon} \quad \text { if } \operatorname{dist}\left(y_{i, \varepsilon}, y_{j, \varepsilon}\right) \leq 10 R_{1},
$$


and denote $\mathcal{C}_{j}, j \in J$ the different equivalence classes. We define $B\left(z_{j}, R_{2, j}\right)$ for each $j \in J$ as the smallest ball such that

$$
\overline{\bigcup_{y_{i, \varepsilon} \in \mathcal{C}_{j}} B\left(y_{i, \varepsilon}, R_{1}\right)} \subset \overline{B\left(z_{j}, R_{2, j}\right)},
$$

and we set $R_{2}:=\max _{j} R_{2, j}$. If $\operatorname{dist}\left(z_{j, \varepsilon}, z_{k, \varepsilon}\right) \geq 10 R_{2}$ for each $j \neq k$ we are done, otherwise we repeat inductively the previous growing argument. Since at each step, the number of equivalence classes decreases at least by one, the process finishes after at most $\sharp I$ steps.

Step 2: Choosing a good unfolding of the torus. Since $(24)$ and $(31)$ are satisfied, we infer from Lemma 2.1 that there exists a good unfolding of the torus $\Pi_{n}$ such that

$$
\int_{\partial \Omega_{n}} e_{\varepsilon}(w) \leq \frac{2^{N-1} M_{0}|\log \varepsilon|}{n} \leq C,
$$

where $C$ does not depend on $n$ or $\varepsilon$. In particular, $\|w\|_{H^{1}\left(\partial \Omega_{n}\right)}$ is uniformly bounded.

Step 3: Uniform $W_{\text {loc }}^{1, p}$ estimates. Let $x_{0} \in \Omega_{n}$ and $1 \leq p<N /(N-1)$ be given. As in the proof of Proposition C.2 C-3 we obtain

$$
\int_{B\left(x_{0}, 1\right)}|\nabla w|^{p} \leq C\left[\int_{B\left(x_{0}, 1\right)}|\nabla \rho|^{p}+\int_{B\left(x_{0}, 1\right)}|w \times \nabla w|^{p}+1\right],
$$

where $\rho:=|w|$ and $C$ depends only on $p, \Lambda_{0}$ and $M_{0}$. The estimate for the modulus is also obtained as in Proposition C.2 by replacing $\Omega$ by $B\left(x_{0}, 1\right)$; we have

$$
\int_{B\left(x_{0}, 1\right)}|\nabla \rho|^{p} \leq C
$$

where $C$ does not depend on $n$ or $\varepsilon$.

Consider the Hodge-de Rham decomposition of $w \times \nabla w$ in $\Omega_{n}$ :

$$
w \times \nabla w=d \varphi+d^{*} \psi
$$

where the function $\varphi$ satisfies $\varphi=0$ on $\partial \Omega_{n}$ and the 2-form $\psi$ satisfies $d \psi=0$ on $\Omega_{n}$ and $\psi_{\top}=0$ on $\partial \Omega_{n}$. Applying respectively the operators $d^{*}$ and $d$ to $\mathrm{C}-19$ we obtain the equation for $\varphi($ resp. $\psi)$ :

$$
\begin{cases}-\Delta \varphi=c(\varepsilon) \frac{\partial}{\partial x_{1}}\left(|w|^{2}-1\right)|\log \varepsilon| & \text { in } \Omega_{n} \\ \varphi=0 & \text { on } \partial \Omega_{n}\end{cases}
$$

and

$$
\begin{cases}-\Delta \psi=2 J w & \text { in } \Omega_{n}, \\ \psi_{\top}=0, \quad\left(d^{*} \psi\right)_{\top}=(w \times d w)_{\top} & \text { on } \partial \Omega_{n} .\end{cases}
$$

Again the estimate for $\varphi$ follows as in Proposition C.2. and we obtain

$$
\int_{B\left(x_{0}, 1\right)}|\nabla \varphi|^{p} \leq C
$$

where $C$ does not depend on $n$ or $\varepsilon$ (and $C \rightarrow 0$ as $\varepsilon \rightarrow 0$ ). 
The estimate for $\psi$ is more delicate since the embedding constants used in the proof of Proposition C.2 heavily depend on $n$. We will overcome this difficulty by taking advantage of the confinement of $J w$ described in Step 1. Let $\tilde{w}$ be defined by

$$
\tilde{w}(x):= \begin{cases}2 w(x) & \text { if }|w(x)| \leq 1 / 2 \\ w(x) /|w(x)| & \text { if }|w(x)| \geq 1 / 2\end{cases}
$$

Notice that $E_{\varepsilon}(\tilde{w}) \leq 4 M_{0}|\log \varepsilon|$ and that $J \tilde{w}$ is supported in $S_{\varepsilon}$. We also define, for $1 \leq i \leq q$, the 2 -forms

$$
\omega_{i}:=2 J \tilde{w}\left\llcorner B\left(x_{i, \varepsilon}, R\right) .\right.
$$

Let $\psi_{0, i}$ be the solution of the problem

$$
\begin{cases}-\Delta \psi_{0, i}=\omega_{i} & \text { in } \Omega_{n} \\ \psi_{0, i}=0 & \text { on } \partial \Omega_{n}\end{cases}
$$

(note the different kind of boundary conditions here). Let $\psi_{1}$ be the solution of

$$
\begin{cases}-\Delta \psi_{1}=2(J w-J \tilde{w}) & \text { in } \Omega_{n}, \\ \left(\psi_{1}\right)_{\top}=0, \quad\left(d^{*} \psi_{2}\right)_{\top}=0 & \text { on } \partial \Omega_{n},\end{cases}
$$

and $\psi_{2}$ the solution of

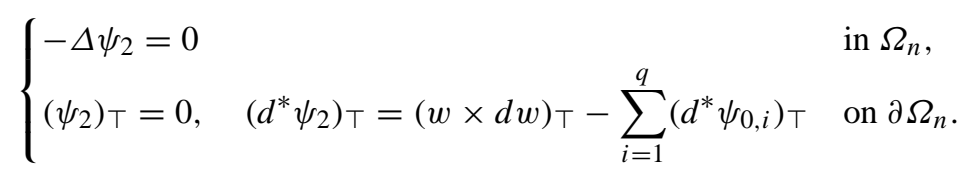

Clearly,

$$
\psi=\sum_{i=1}^{q} \psi_{0, i}+\psi_{1}+\psi_{2}
$$

We also set

$$
U_{1}^{i}:=B\left(x_{0}, 1\right) \cap\left(\Omega_{n} \backslash B\left(x_{i, \varepsilon}, 2 R\right)\right), \quad U_{2}^{i}:=\overline{B\left(x_{i, \varepsilon}, 2 R\right)} .
$$

Estimate for $\psi_{0, i}$. From the Green formula

$$
\psi_{0, i}(x)=\int_{\operatorname{supp}\left(\omega_{i}\right)}\left\langle\omega_{i}(x), G_{\Omega_{n}}(x, y)\right\rangle d y
$$

we deduce that

$$
\left\|\psi_{0, i}\right\|_{\mathcal{C}^{k}\left(U_{1}^{i}\right)} \leq C(k)\left\|\omega_{i}\right\|_{\left[\mathcal{C}^{0, \alpha}\left(U_{1}^{i}\right)\right]^{*}} \leq C(k) .
$$

Indeed, for any $x$ in $U_{1}^{i}$ and $y \in \operatorname{supp}\left(\omega_{i}\right)$ one has

$$
\min \left(\operatorname{dist}\left(x, \partial \Omega_{n}\right), \operatorname{dist}\left(y, \partial \Omega_{n}\right), \operatorname{dist}(x, y)\right) \geq R,
$$

so that $\mathrm{C}-27$ follows from standard estimates on the Green functions (which is even explicit in the case of the cube $\Omega_{n}$ ). 
For $U_{2}^{i}$, consider the solution $\tilde{\psi}_{0, i}$ of

$$
\begin{cases}-\Delta \tilde{\psi}_{0, i}=\omega_{i} & \text { in } B\left(x_{i, \varepsilon}, 3 R\right) \\ \tilde{\psi}_{0, i}=0 & \text { on } \partial B\left(x_{i, \varepsilon}, 3 R\right) .\end{cases}
$$

Following the lines of Proposition C.2 we obtain

$$
\int_{B\left(x_{i, \varepsilon}, 3 R\right)}\left|\nabla \tilde{\psi}_{0, i}\right|^{p} \leq C(R)\left\|\omega_{i}\right\|_{\left[\mathcal{C}^{0, \alpha}\right]^{*}} \leq C\left(R, M_{0}\right) .
$$

On the other hand, for $x \in U_{2}^{i}$ we have

$$
\tilde{\psi}_{0, i}(x)-\psi_{0, i}(x)=\int_{\operatorname{supp}\left(\omega_{i}\right)}\left\langle\omega_{i}(y),\left[R_{B\left(x_{i, \varepsilon}, 3 R\right)}(x, y)-R_{\Omega_{n}}(x, y)\right]\right\rangle d y,
$$

where $R_{\Omega_{n}}$ stands for the regular part of the Green function $G_{\Omega_{n}}$ and similarly for $B\left(x_{i, \varepsilon}, 3 R\right)$. Note that for all $x \in U_{2}^{i}$ and for all $y \in \operatorname{supp}\left(\omega_{i}\right)$,

$$
\min \left(\mathrm{d}\left(x, \partial \Omega_{n}\right), \mathrm{d}\left(y, \partial \Omega_{n}\right), \mathrm{d}\left(x, \partial B\left(x_{i, \varepsilon}, 3 R\right)\right), \mathrm{d}\left(y, \partial B\left(x_{i, \varepsilon}, 3 R\right)\right)\right) \geq R,
$$

so that again using standard estimates

$$
\left\|\tilde{\psi}_{0, i}-\psi_{0, i}\right\|_{\mathcal{C}^{k}\left(U_{2}^{i}\right)} \leq C(k)\left\|\omega_{i}\right\|_{\left[\mathcal{C}^{0, \alpha}\right]^{*}} \leq C\left(R, M_{0}\right) .
$$

Combining (C-27), C-29 and (C-30 we obtain

$$
\int_{B\left(x_{0}, 1\right)}\left|\nabla \psi_{0, i}\right|^{p} \leq C
$$

where $C$ does not depend on $n$ or $\varepsilon$.

Estimate for $\psi_{1}$. From standard elliptic estimates we have

$$
\left\|\psi_{1}\right\|_{W_{0}^{1, p}\left(\Omega_{n}\right)} \leq C\|J w-J \tilde{w}\|_{\left[W_{0}^{1, p^{\prime}}\left(\Omega_{n}\right)\right]^{*}},
$$

where $C$ does not depend on $n$ (indeed the previous inequality is invariant under scaling of the domain and of the corresponding equation). On the other hand,

$$
\|J w-J \tilde{w}\|_{\left[W_{0}^{1, p}\left(\Omega_{n}\right)\right]^{*}}=\sup _{h \in W_{0}^{1, q}\left(\Omega_{n}, \Lambda^{2} \mathbb{R}^{N}\right),\|h\|=1} \int_{\Omega_{n}}\langle J w-J \tilde{w}, h\rangle,
$$

and

$$
\begin{aligned}
\int_{\Omega_{n}}\langle J w-J \tilde{w}, h\rangle & =\int_{\Omega_{n}}\left\langle w \times d w-\tilde{w} \times d \tilde{w}, d^{*} h\right\rangle \\
& \leq C\left(\int_{\Omega_{n}}|\nabla h|^{q}\right)^{1 / q} \cdot\left(\int_{\Omega_{n}}|w \times d w-\tilde{w} \times d \tilde{w}|^{p}\right)^{1 / p} .
\end{aligned}
$$

On $S_{\varepsilon}$

$$
|w \times d w-\tilde{w} \times d \tilde{w}| \leq C|w| \cdot|\nabla w|
$$


so that since $\left|S_{\varepsilon}\right| \leq C \varepsilon^{2}|\log \varepsilon|$,

$$
\begin{aligned}
\left(\int_{S_{\varepsilon}}|w \times d w-\tilde{w} \times d \tilde{w}|^{p}\right)^{1 / p} & \leq C\left(\int_{S_{\varepsilon}}|\nabla w|^{2}\right)^{1 / 2} \cdot\left(\int_{S_{\varepsilon}}|w|^{s}\right)^{1 / s} \\
& \leq C|\log \varepsilon|^{1 / 2}\left(\varepsilon^{2}|\log \varepsilon|\right)^{1 / s},
\end{aligned}
$$

where $s:=2 p /(2-p) \geq 2$. Outside $S_{\varepsilon}$, we have

$$
|w \times d w-\tilde{w} \times d \tilde{w}|=\left|\frac{|w|^{2}-1}{|w|^{2}} w \times d w\right| \leq\left. 4|| w\right|^{2}-1|\cdot| \nabla w \mid
$$

so that

$$
\begin{aligned}
\left(\int_{\Omega_{n} \backslash S_{\varepsilon}}|w \times d w-\tilde{w} \times d \tilde{w}|^{p}\right)^{1 / p} & \leq C\left(\int_{\Omega_{n} \backslash S_{\varepsilon}}|\nabla w|^{2}\right)^{1 / 2} \cdot\left(\int_{\Omega_{n} \backslash S_{\varepsilon}}\left(|w|^{2}-1\right)^{s}\right)^{1 / s} \\
& \leq C \varepsilon^{2 / s}|\log \varepsilon|^{1 / 2}\left(\int_{\Omega_{n} \backslash S_{\varepsilon}} \frac{\left(|w|^{2}-1\right)^{2}}{\varepsilon^{2}}\right)^{1 / s} \\
& \leq C \varepsilon^{2 / s}|\log \varepsilon|^{1 / 2}|\log \varepsilon|^{1 / s} \leq C .
\end{aligned}
$$

Combining these two estimates with $\mathrm{C}-31$ we thus obtain

$$
\int_{B\left(x_{0}, 1\right)}\left|\nabla \psi_{1}\right|^{p} \leq C
$$

where $C$ does not depend on $n$ or $\varepsilon$.

Estimate for $\psi_{2}$. We deduce from Step 2 that

$$
\|(w \times d w) \top\|_{L^{2}\left(\partial \Omega_{n}\right)} \leq C .
$$

On the other hand, since $\operatorname{dist}\left(\partial \Omega_{n}, \operatorname{supp}\left(\omega_{i}\right)\right) \geq R$ we have

$$
\left\|\nabla \psi_{0, i}\right\|_{L^{\infty}\left(\partial \Omega_{n}\right)} \leq C
$$

(this again follows from standard estimates on the Green function for the cube $\Omega_{n}$ ). Since $\psi_{2}$ is harmonic on $\Omega_{n}$, we thus obtain

$$
\left\|\psi_{2}\right\|_{\mathcal{C}^{k}\left(B\left(x_{0}, 1\right)\right)} \leq C
$$

where $C$ depends on $k$ but not on $n$ or $\varepsilon$.

Combining the estimates for $\psi_{0, i}, \psi_{1}$ and $\psi_{2}$ with $(\mathrm{C}-17)$ and $(\mathrm{C}-18)$ we conclude that

$$
\int_{B\left(x_{0}, 1\right)}|\nabla w|^{p} \leq C .
$$

This establishes claim (i) of the Theorem.

Next, we prove estimate (ii) of the Theorem, i.e. provide uniform energy bounds away from the bad balls. Here, we will work directly on $\Pi_{n}$ (as a manifold). Therefore, the Hodge-de Rham decomposition will involve also harmonic forms. The next step will be useful to control these forms. 
Step 4: Degree estimate. Since $|w| \geq 1 / 2$ off $S_{\varepsilon}$, we may write $w(x)=\rho(x) \exp (i \varphi(x))$ off $S_{\varepsilon}$, where $\varphi(x) \in S^{1}$. Moreover, since

$$
\tilde{\Omega}_{n}:=\Omega_{n} \backslash \bigcup_{i=1}^{q} B\left(x_{i, \varepsilon}, R\right)
$$

is simply connected, the phase $\varphi(x)$ can be lifted as a function from $\tilde{\Omega}_{n}$ to $\mathbb{R}$. If the coordinates $\left(y_{2}, \ldots, y_{N}\right)$ are such that

$$
[-n, n] \times\left(y_{2}, \ldots, y_{N}\right) \cap \bigcup_{i=1}^{q} B\left(x_{i, \varepsilon}, R\right)=\emptyset
$$

then the degree of the map $s \mapsto \varphi\left(s, y_{2}, \ldots, y_{n}\right)$, i.e.

$$
d:=\operatorname{deg}\left(s \mapsto \varphi\left(s, y_{2}, \ldots, y_{n}\right)\right)
$$

is well defined. Clearly it follows from the invariance of the degree under homotopy that $d$ does not depend on the particular choice of an admissible $\left(y_{2}, \ldots, y_{N}\right)$.

We claim that $d=0$ (an elementary way to rephrase this is that the lifted phase $\varphi$ takes the same values on opposite faces of $\Omega_{n}$ ). Indeed, from Step 1 we infer that the set of admissible $\left(y_{2}, \ldots, y_{N}\right) \in[-n, n]^{N-1}$ has measure larger than $n^{N-1}$ for $n$ sufficiently large (and thus $\varepsilon$ sufficiently small).

On the other hand, if $d \neq 0$ we obtain for each admissible $\left(y_{2}, \ldots, y_{N}\right)$,

$$
\int_{-n}^{n}\left|\nabla w\left(s, y_{2}, \ldots, y_{N}\right)\right|^{2} d s \geq \frac{1}{4} \int_{-n}^{n}\left|\frac{\partial \varphi}{\partial x_{1}}\left(s, y_{2}, \ldots, y_{N}\right)\right|^{2} d s \geq 2 n \frac{1}{4}\left(\frac{2 \pi}{2 n}\right)^{2}=\frac{\pi^{2}}{2 n},
$$

so that by Fubini's theorem,

$$
\int_{\Omega_{n}}|\nabla w|^{2} \geq n^{N-1} \frac{\pi^{2}}{2 n}=\frac{\pi^{2}}{2} n^{N-2} \geq 2\left(M_{0}+1\right)|\log \varepsilon| .
$$

This contradicts hypothesis 24 and proves the claim. Obviously the corresponding degree computed with respect to the other coordinates is also zero.

Step 5: Local uniform energy estimates. Let $x \in \Omega_{n}$ and $r>0$ be such that $B(x, r) \subset$ $\Omega_{n} \backslash S_{\varepsilon}$. As in the previous step, we write $w(x)=\rho(x) \exp (i \varphi(x))$ in $B(x, r)$, and we have

$$
\operatorname{div}\left(\rho^{2} \nabla \varphi\right)=c|\log \varepsilon| \frac{\partial}{\partial x_{1}}\left(\rho^{2}-1\right)
$$

Let $\tilde{\varphi}$ be the solution of

$$
\begin{cases}\operatorname{div}\left(\rho^{2} \nabla \tilde{\varphi}\right)=c|\log \varepsilon| \frac{\partial}{\partial x_{1}}\left(\rho^{2}-1\right) & \text { in } B(x, r), \\ \tilde{\varphi}=0 & \text { on } \partial B(x, r) .\end{cases}
$$

Multiplying $\mathrm{C}-35$ by $\tilde{\varphi}$ and integrating by parts leads to

$$
\int_{B(x, r)}|\nabla \tilde{\varphi}|^{2} \leq C \varepsilon^{2}|\log \varepsilon| \leq C .
$$

On the other hand, $\bar{\varphi}:=\varphi-\tilde{\varphi}$ satisfies

$$
\operatorname{div}\left(\rho^{2} \nabla \bar{\varphi}\right)=0 \quad \text { on } B(x, r) .
$$


Since $\varphi$ is defined up to a constant multiple of $2 \pi$, we may assume without loss of generality that

$$
\frac{1}{|B(x, r)|} \int_{B(x, r)} \bar{\varphi} \in[0,2 \pi) .
$$

Combining $\left(\mathrm{C}-37\right.$ with $\mathrm{C}-38$ and the $W_{\text {loc }}^{1, p}$ estimates in Step 4 we obtain, using standard elliptic regularity theory,

$$
\int_{B(x, r / 2)}|\nabla \bar{\varphi}|^{2} \leq C
$$

so that finally by (C-36),

$$
\int_{B(x, r / 2)}|\nabla \varphi|^{2} \leq C
$$

Next, let $\xi \in \mathcal{D}(B(x, r / 2)), 0 \leq \xi \leq 1$, be such that $\xi \equiv 1$ on $B(x, r / 4)$. Multiplying the equation

$$
\Delta \rho-\rho|\nabla \varphi|^{2}+\frac{1}{\varepsilon^{2}} \rho\left(1-\rho^{2}\right)=-c|\log \varepsilon| \rho \frac{\partial \varphi}{\partial x_{1}}
$$

by $\left(1-\rho^{2}\right) \xi^{2}$ and integrating by parts we obtain

$$
\begin{aligned}
\int_{B(x, r / 2)}\left(2 \rho|\nabla \rho|^{2} \xi^{2}+\rho \frac{\left(1-\rho^{2}\right)^{2}}{\varepsilon^{2}}\right)=\int_{B(x, r / 2)} 2 \xi\left(1-\rho^{2}\right) \nabla \rho \cdot \nabla \xi \\
+\int_{B(x, r / 2)}\left(\rho\left(1-\rho^{2}\right) \xi^{2}|\nabla \varphi|^{2}-c|\log \varepsilon| \rho \frac{\partial \varphi}{\partial x_{1}}\left(1-\rho^{2}\right) \xi^{2}\right) .
\end{aligned}
$$

On the other hand, we have

$$
\int_{B(x, r / 2)} 2 \xi\left(1-\rho^{2}\right) \nabla \rho \cdot \nabla \xi \leq \frac{1}{10} \int_{B(x, r / 2)}|\nabla \rho|^{2} \xi^{2}+10 \int_{B(x, r / 2)}\left(1-\rho^{2}\right)^{2}|\nabla \xi|^{2},
$$

and from $\mathrm{C}-40$,

$$
\int_{B(x, r / 2)} \rho\left(1-\rho^{2}\right) \xi^{2}|\nabla \varphi|^{2} \leq C
$$

and

$$
\begin{aligned}
& \int_{B(x, r / 2)} c|\log \varepsilon| \rho \frac{\partial \varphi}{\partial x_{1}}\left(1-\rho^{2}\right) \xi^{2} \\
& \leq C\left(\int_{B(x, r / 2)}|\nabla \varphi|^{2}\right)^{1 / 2}\left(\int_{B(x, r / 2)} \frac{\left(1-\rho^{2}\right)^{2}}{\varepsilon^{2}}\right) \varepsilon|\log \varepsilon| \leq C .
\end{aligned}
$$

Hence, from C-42 and since $\rho \geq 1 / 2$ on $B(x, r)$,

$$
\int_{B(x, r / 4)}\left(|\nabla \rho|^{2}+\frac{\left(1-\rho^{2}\right)^{2}}{4 \varepsilon^{2}}\right) \leq C,
$$

which, combined with C-40 leads to

$$
\int_{B(x, r / 4)} e_{\varepsilon}(w) \leq C .
$$


Step 6: Proof of estimate (ii). In order to conclude the proof of Theorem 4 it remains to show that

$$
\int_{\Omega_{n} \backslash \bigcup_{i=1}^{q} B\left(x_{i, \varepsilon}, R\right)} e_{\varepsilon}(w) \leq C .
$$

As in Proposition C.2, we have

$$
\int_{\Pi_{n} \backslash \bigcup_{i=1}^{q} B\left(x_{i, \varepsilon}, R\right)}|\nabla w|^{2} \leq C\left(1+\int_{\Pi_{n} \backslash \bigcup_{i=1}^{q} B\left(x_{i, \varepsilon}, R\right)}\left(|\nabla \rho|^{2}+|w \times d w|^{2}\right)\right) .
$$

Here we consider the Hodge-de Rham decomposition of $w \times \nabla w$ in $\Pi_{n}$ (as a manifold):

$$
w \times \nabla w=d \varphi+d^{*} \psi+\sum_{i=1}^{N} \alpha_{i} d x_{i}
$$

where the 2-form $\psi$ satisfies $d \psi=0$ on $\Pi_{n}$, each $\alpha_{i}$ is a real number and the $d x_{i}$ represent the canonical harmonic 1-forms on $\Pi_{n}$. Applying respectively the operators $d^{*}$ and $d$ to $(\mathrm{C}-46)$ we obtain the equation for $\varphi(\operatorname{resp} . \psi)$ :

$$
-\Delta \varphi=c(\varepsilon) \frac{\partial}{\partial x_{1}}\left(\rho^{2}-1\right)|\log \varepsilon| \quad \text { in } \Pi_{n}
$$

and

$$
-\Delta \psi=2 J w \quad \text { in } \Pi_{n} .
$$

Again the estimate for $\varphi$ follows as in Proposition C.2 (C-13), and we obtain

$$
\int_{\Pi_{n}}|\nabla \varphi|^{2} \leq C
$$

where $C$ does not depend on $n$ or $\varepsilon$ (and $C \rightarrow 0$ as $\varepsilon \rightarrow 0$ ).

The estimate for $\psi$ has to be slightly adapted with respect to Step 3 . Let $\tilde{w}$ be defined by

$$
\tilde{w}(x):= \begin{cases}w(x) & \text { if } x \in \bigcup_{i=1}^{q} B\left(x_{i, \varepsilon}, R / 2\right), \\ (4 s / R-2) w(x)+(3-4 s / R) w(x) /|w(x)| & \\ w(x) /|w(x)| & \text { if } s:=\operatorname{dist}\left(x, \bigcup_{i}\left\{x_{i, \varepsilon}\right\}\right) \in(R / 2,3 R / 4), \\ \text { otherwise. }\end{cases}
$$

Notice that $E_{\varepsilon}(\tilde{w}) \leq C M_{0}|\log \varepsilon|$ and that $J \tilde{w}$ is supported in the set $\bigcup_{i=1}^{q} B\left(x_{i, \varepsilon}, 3 R / 4\right)$. We also have

$$
w \times d w=\tilde{w} \times d \tilde{w} \quad \text { on } \bigcup_{i=1}^{q} B\left(x_{i, \varepsilon}, R / 2\right) .
$$

We also define, for $1 \leq i \leq q$, the 2 -forms

$$
\omega_{i}:=2 J \tilde{w}\left\llcorner B\left(x_{i, \varepsilon}, 3 R / 4\right),\right.
$$

and denote by $\psi_{0, i}$ the Newtonian potential of $\omega_{i}$ on $\Pi_{n}$ (i.e. $\psi_{0, i}:=G_{n} * \omega_{i}$, where $G_{n}$ is the Green function on $\Pi_{n}$.) Similarly, $\psi_{1}$ denotes the Newtonian potential of $2(J w-J \tilde{w})$ on $\Pi_{n}$. Clearly,

$$
\psi(x)=\sum_{i=1}^{q} \psi_{0, i}(x)+\psi_{1}(x) .
$$


We claim that

$$
\left|\nabla \psi_{0, i}(x)\right| \leq C\left(\operatorname{dist}\left(x, x_{i, \varepsilon}\right)\right)^{1-N} \quad \forall x \in \Pi_{n} \backslash B\left(x_{i, \varepsilon}, R\right),
$$

where $C$ does not depend on $n$ or $\varepsilon$. Indeed, this is a direct consequence of the formula

$$
\nabla \psi_{0, i}(x)=\int_{\Pi_{n}} \frac{\partial G}{\partial y}(x, y) \omega_{i}(y) d y,
$$

of the $\left[\mathcal{C}^{0, \alpha}\right]^{*}$ uniform bound on $\omega_{i}$, and of classical estimates on $G_{n}$. Hence, since $N \geq 3$, we obtain

$$
\int_{\Pi_{n} \backslash \bigcup_{i=1}^{q} B\left(x_{i, \varepsilon}, R\right)}\left|\nabla \psi_{0, i}\right|^{2} \leq \int_{\mathbb{R}^{N} \backslash B(0, R)} C|x|^{2-2 N} d x \leq C .
$$

We next turn to the estimate for $\psi_{1}$. We have

$$
\left\|\nabla \psi_{1}\right\|_{L^{2}\left(\Pi_{n}\right)} \leq C \sup _{h \in \mathcal{C}^{\infty}\left(\Pi_{n}, \Lambda^{2} \mathbb{R}^{N}\right)}\left\{\int_{\Pi_{n}}\langle J w-J \tilde{w}, h\rangle: \int_{\Pi_{n}}|\nabla h|^{2}=1\right\} .
$$

On the other hand, taking $\mathrm{C}-50$ into account, we obtain

$$
\int_{\Pi_{n}}\langle J w-J \tilde{w}, h\rangle=\int_{\Pi_{n}}\left\langle w \times d w-\tilde{w} \times d \tilde{w}, d^{*} h\right\rangle=\int_{\tilde{\Pi}_{n}}\left\langle w \times d w-\tilde{w} \times d \tilde{w}, d^{*} h\right\rangle
$$

where $\tilde{\Pi}_{n}:=\Pi_{n} \backslash \bigcup_{i=1}^{q} B\left(x_{i, \varepsilon}, R / 2\right)$. Notice that $|w| \geq 1 / 2$ in $\tilde{\Pi}_{n}$, hence

$$
\int_{\tilde{\Pi}_{n}}\left\langle w \times d w-\tilde{w} \times d \tilde{w}, d^{*} h\right\rangle \leq C\left\|\rho^{2}-1\right\|_{L^{\infty}\left(\tilde{\Pi}_{n}\right)}\left(\int_{\tilde{\Pi}_{n}}|\nabla w|^{2}\right)^{1 / 2}\left(\int_{\tilde{\Pi}_{n}}|\nabla h|^{2}\right)^{1 / 2} .
$$

From Theorem 3 we know that $|w|$ uniformly converges to 1 on $\tilde{\Pi}_{n}$ (and actually uniformly with respect to $n$ as can be seen by examining Step 2 of the proof of Theorem 3). Hence, we obtain

$$
\left\|\nabla \psi_{1}\right\|_{L^{2}\left(\Pi_{n}\right)} \leq C\left(1+r(\varepsilon) \int_{\tilde{\Pi}_{n}}|\nabla w|^{2}\right),
$$

where $r(\varepsilon) \rightarrow 0$ as $\varepsilon \rightarrow 0$, uniformly in $n$.

Finally, we turn to the components of the harmonic forms. We claim that

$$
\left|\alpha_{i}\right| \leq \frac{C}{\left|\Pi_{n}\right|^{2}}
$$

Indeed, since

$$
\alpha_{i}=\frac{1}{\left|\Pi_{n}\right|^{2}} \int_{\Pi_{n}}\left\langle w \times d w, d x_{i}\right\rangle
$$

it suffices to prove that

$$
\left|\int_{\Pi_{n}}\left\langle w \times d w, d x_{i}\right\rangle\right| \leq C
$$


Let $R^{\prime} \in[R / 2, R]$ and $\tilde{\Pi}_{n}^{\prime}:=\Pi_{n} \backslash \bigcup_{i=1}^{q} B\left(x_{i, \varepsilon}, R^{\prime}\right)$. The phase $\varphi$ of $w$ is well defined in $\tilde{\Pi}_{n}^{\prime}$; we extend it as a continuous function $\varphi^{\prime}$ on $\Pi_{n}$ by considering its harmonic extension inside each ball $B\left(x_{i, \varepsilon}, R^{\prime}\right)$. We have

$$
\begin{aligned}
\left|\int_{\Pi_{n}}\left\langle w \times d w, d x_{i}\right\rangle\right| \leq & \left|\int_{\bigcup_{i=1}^{q} B\left(x_{i, \varepsilon}, R^{\prime}\right)}\left\langle w \times d w, d x_{i}\right\rangle\right|+\left|\int_{\tilde{\Pi}_{n}^{\prime}} \rho^{2} \frac{\partial \varphi}{\partial x_{1}}\right| \\
\leq & C \int_{\bigcup_{i=1}^{q} B\left(x_{i, \varepsilon}, R^{\prime}\right)}|\nabla w|+C\left|\int_{\tilde{\Pi}_{n}^{\prime}}\left(\rho^{2}-1\right)\right| \nabla \varphi|| \\
& +C\left|\int_{\Pi_{n}} \frac{\partial \varphi^{\prime}}{\partial x_{1}}\right|+\int_{\bigcup_{i=1}^{q} B\left(x_{i, \varepsilon}, R^{\prime}\right)}\left|\nabla \varphi^{\prime}\right| .
\end{aligned}
$$

From Step 3 we infer that

$$
\int_{\bigcup_{i=1}^{q} B\left(x_{i, \varepsilon}, R^{\prime}\right)}|\nabla w| \leq C .
$$

An averaging argument shows that there exists $R^{\prime} \in[R / 2, R]$ such that

$$
\int_{\bigcup_{i=1}^{q} B\left(x_{i, \varepsilon}, R^{\prime}\right)}\left|\nabla \varphi^{\prime}\right| \leq C\left(\int_{\bigcup_{i=1}^{q} B\left(x_{i, \varepsilon}, R^{\prime}\right)}\left|\nabla \varphi^{\prime}\right|^{2}\right)^{1 / 2} \leq C\left(\int_{\bigcup_{i=1}^{q} B\left(x_{i, \varepsilon}, R\right) \cap \tilde{\Pi}_{n}}|\nabla w|^{2}\right)^{1 / 2}
$$

so that by Step 5,

$$
\int_{\bigcup_{i=1}^{q} B\left(x_{i, \varepsilon}, R^{\prime}\right)}\left|\nabla \varphi^{\prime}\right| \leq C .
$$

We also have

$$
\left|\int_{\tilde{\Pi}_{n}^{\prime}}\left(\rho^{2}-1\right)\right| \nabla \varphi|| \leq C \varepsilon|\log \varepsilon| \leq C
$$

and by Step 4 ,

$$
\int_{\Pi_{n}} \frac{\partial \varphi^{\prime}}{\partial x_{1}}=0
$$

This proves the claim. Coming back to $w \times d w$, combining $(\overline{\mathrm{C}}-47 \overrightarrow{\mathrm{C}}, \overline{\mathrm{C}-52}),(\mathrm{C}-53)$ and the previous claim, we obtain

$$
\int_{\Pi_{n} \backslash \bigcup_{i=1}^{q} B\left(x_{i, \varepsilon}, R\right)}|w \times d w|^{2} \leq C\left(1+r(\varepsilon) \int_{\tilde{\Pi}_{n}}|\nabla w|^{2}\right),
$$

where $r(\varepsilon) \rightarrow 0$ when $\varepsilon \rightarrow 0$, uniformly in $n$.

We still need the estimate for the modulus. Let $\xi \in \mathcal{D}\left(\tilde{\Pi}_{n}\right), 0 \leq \xi \leq 1$, be such that $\xi \equiv 1$ on $\Pi_{n} \backslash \bigcup_{i=1}^{q} B\left(x_{i, \varepsilon}, R\right)$. Multiplying the equation

$$
\Delta \rho-\rho|\nabla \varphi|^{2}+\frac{1}{\varepsilon^{2}} \rho\left(1-\rho^{2}\right)=-c|\log \varepsilon| \rho \frac{\partial \varphi}{\partial x_{1}}
$$


by $\left(1-\rho^{2}\right) \xi^{2}$ and integrating by parts we obtain

$$
\begin{aligned}
\int_{\tilde{\Pi}_{n}} 2 \rho|\nabla \rho|^{2} \xi^{2}+ & \rho \frac{\left(1-\rho^{2}\right)^{2}}{\varepsilon^{2}}=\int_{\tilde{\Pi}_{n}} 2 \xi\left(1-\rho^{2}\right) \nabla \rho \cdot \nabla \xi \\
& +\int_{\tilde{\Pi}_{n}}\left(\rho\left(1-\rho^{2}\right) \xi^{2}|\nabla \varphi|^{2}-c|\log \varepsilon| \rho \frac{\partial \varphi}{\partial x_{1}}\left(1-\rho^{2}\right) \xi^{2}\right) .
\end{aligned}
$$

Arguing as in Step 5, we deduce from $(\mathrm{C}-56$ that

$$
\int_{\Pi_{n} \backslash \bigcup_{i=1}^{q} B\left(x_{i, \varepsilon}, R\right)}|\nabla \rho|^{2}+\frac{\left(1-\rho^{2}\right)^{2}}{4 \varepsilon^{2}} \leq C\left(1+r(\varepsilon) \int_{\tilde{\Pi}_{n}}|\nabla w|^{2}\right),
$$

where $r(\varepsilon) \rightarrow 0$ when $\varepsilon \rightarrow 0$, uniformly in $n$.

We can now complete the proof. Adding (C-55) to (C-57) we obtain, using (C-45) and Step 5,

$$
\begin{aligned}
\int_{\Pi_{n} \backslash \bigcup_{i=1}^{q} B\left(x_{i, \varepsilon}, R\right)} e_{\varepsilon}(w) & \leq C\left(1+r(\varepsilon) \int_{\tilde{\Pi}_{n}}|\nabla w|^{2}\right) \\
& \leq C\left(1+r(\varepsilon) \int_{\Pi_{n} \backslash \bigcup_{i=1}^{q} B\left(x_{i, \varepsilon}, R\right)}|\nabla w|^{2}\right) .
\end{aligned}
$$

For $\varepsilon \leq \varepsilon_{0}$ sufficiently small, $\operatorname{Cr}(\varepsilon)<1 / 2$, which yields the desired estimate

$$
\int_{\Pi_{n} \backslash \bigcup_{i=1}^{q} B\left(x_{i, \varepsilon}, R\right)} e_{\varepsilon}(w) \leq C .
$$

For $\varepsilon \geq \varepsilon_{0}$ the previous inequality is clearly also satisfied, and the proof is complete.

Acknowledgements. We would like to thank Giovanni Alberti, Thierry Cazenave, Thierry De Pauw, Jean-Claude Saut and Jan Bouwe van den Berg for useful discussions. This work was partially supported by European RTN Grant HPRN-CT-2002-00274 "Front, Singularities".

\section{References}

[1] Alberti, G., Baldo, S., Orlandi, G.: Variational convergence for functionals of GinzburgLandau type. Preprint (2002)

[2] Allard, W.: On the first variation of a varifold. Ann. of Math. 95, 417-491 (1972) Zbl 0252.49028 MR 46\#6136

[3] Almgren, F.: Optimal isoperimetric inequalities. Indiana Univ. Math. J. 35, 451-547 (1986) Zbl 0585.49030 MR 88c:49032

[4] Ambrosetti, A., Struwe, M.: Existence of steady vortex rings in an ideal fluid. Arch. Rat. Mech. Anal. 108, 97-109 (1989) Zbl 0694.76012 MR 90g:76036

[5] Ambrosio, L., Soner, M.: A measure theoretic approach to higher codimension mean curvature flow. Ann. Scuola Norm. Sup. Pisa Cl. Sci. 25, 27-49 (1997) Zbl pre01766596 MR 99m:35217

[6] Benjamin, T. B.: The stability of solitary waves. Proc. Roy. Soc. London Ser. A 328, 153-183 (1972) MR 49 \#3348

[7] Bethuel, F., Bourgain, J., Brezis, H., Orlandi, G.: $W^{1, p}$ estimates for solutions to the Ginzburg-Landau functional with boundary data in $H^{1 / 2}$. C. R. Acad. Sci. Paris Sér. I Math. 333, 1-8 (2001) Zbl pre01719347 
[8] Bethuel, F., Brezis, H., Hélein, F.: Asymptotics for the minimizers of a Ginzburg-Landau functional. Calc. Var. PDE 1, 123-148 (1993) Zbl 0834.35014

[9] Bethuel, F., Brezis, H., Hélein, F.: Ginzburg-Landau Vortices. Birkhäuser, Boston (1994) Zbl 0802.35142 MR 95c:58044

[10] Bethuel, F., Brezis, H., Orlandi, G.: Asymptotics for the Ginzburg-Landau equation in arbitrary dimensions. J. Funct. Anal. 186, 432-520 (2001). Erratum 188, 548-549 (2002) Zbl pre01703083 MR 2002k:35081a MR 2002k:35081b

[11] Bethuel, F., Orlandi, G.: Uniform estimates for the parabolic Ginzburg-Landau equation. ESAIM Control Optim. Calc. Var. 8, 219-238 (2002) MR 1932951

[12] Bethuel, F., Orlandi, G., Smets, D.: Convergence of the parabolic Ginzburg-Landau equation to motion by mean curvature. C. R. Acad. Sci. Paris Sér. I Math. 336, 217-222 (2003), and preprint R03004 Lab. J. L. Lions (2003).

[13] Bethuel, F., Rivière, T.: A minimization problem related to superconductivity. Ann. Inst. H. Poincaré Anal. Non Linéaire 12, 243-303 (1995) Zbl 0842.35119 MR 96g:35045

[14] Bethuel, F., Saut, J.-C.: Traveling waves for the Gross-Pitaevskii equation. Ann. Inst. H. Poincaré Phys. Théor. 70, 147-238 (1999) MR 2000d:35211

[15] Bona, J.: On the stability theory of solitary waves. Proc. Roy. Soc. London Ser. A 344, 363-374 (1975) Zbl 0328.76016 MR 52 \#7292

[16] Bourgain, J., Brezis, H., Mironescu, P.: On the structure of the Sobolev space $H^{1 / 2}$ with values into the circle. C. R. Acad. Sci. Paris Sér. I Math. 331, 119-124 (2000), and detailed paper in preparation.

[17] Brezis, H., Mironescu, P.: Sur une conjecture de E. De Giorgi relative à l'énergie de Ginzburg-Landau. C. R. Acad. Sci. Paris Sér. I Math. 319, 167-170 (1994) Zbl 0805.49004 MR 95h:49016

[18] Cazenave, T., Lions, P.-L.: Orbital stability of standing waves for some nonlinear Schrödinger equations. Comm. Math. Phys. 85, 549-561 (1982) Zbl 0513.35007 MR 84i:81015

[19] Farina, A.: From Ginzburg-Landau to Gross-Pitaevskii. Preprint (2002)

[20] Federer, H.: Geometric Measure Theory. Springer, Berlin (1969) Zbl 0176.00801 MR 41 \#1976

[21] Fetter, A. L., Svidzinsky, A. A.: Vortices in a trapped dilute Bose-Einstein condensate. J. Phys.: Condens. Matter 13, R135-R194 (2001)

[22] Fraenkel, L. E., Berger, M. S.: A global theory of steady vortex rings in an ideal fluid. Acta Math. 132, 13-51 (1974) Zbl 0282.76014 MR 54 \#10901

[23] Giaquinta, M.: Multiple Integrals in the Calculus of Variations and Nonlinear Elliptic Systems. Princeton Univ. Press (1983) Zbl 0516.49003 MR 86b:49003

[24] Ginibre, J., Velo, G.: The Cauchy problem in local spaces for the complex Ginzburg-Landau equation II. Contraction methods. Comm. Math. Phys. 187, 45-79 (1997) Zbl 0889.35046 MR 98i:35174

[25] Gravejat, P.: Decay for travelling waves in the Gross-Pitaevskii equation. Preprint

[26] Grillakis, M., Shatah, J., Strauss, W.: Stability theory of solitary waves in the presence of symmetry. I and II. J. Funct. Anal. 74, 160-197 (1987) and 94, 308-348 (1990) Zbl 0656.35122 Zbl 0711.58013 MR 88g:35169 MR 92a:35135

[27] Helmholtz, H.: Über Integrale der hydrodynamischen Gleichungen, welche den Wirbelwegungen entsprechen. J. Reine Angew. Math. 55, 25-55 (1858)

[28] Jerrard, R. L.: Vortex filament dynamics for Gross-Pitaevsky like equations, I. Preprint

[29] Jerrard, R. L., Soner, H. M.: The Jacobian and the Ginzburg-Landau energy. Calc. Var. PDE 14, 151-191 (2002) Zbl pre01756808 MR 2003d:35069

[30] Jones, C. A., Roberts, P. H.: Motions in a Bose condensate IV. Axisymmetric solitary waves, J. Phys. A 15, 2599-2619 (1982)

[31] Lin, F. H., Rivière, T.: Complex Ginzburg-Landau equation in high dimensions and codimension two area minimizing currents. J. Eur. Math. Soc. 1, 237-311 (1999). Erratum, 2, 87-91 (2000) Zbl 0939.35056 MR 2000g:49048

[32] Lin, F. H., Rivière, T.: A quantization property for static Ginzburg-Landau vortices. Comm. Pure Appl. Math. 54, 206-228 (2001) Zbl pre01592012 MR 2001k:35097 
[33] Moore, D. W., Pullin, D. I.: On steady compressible flows with compact vorticity; the compressible Hill's spherical vortex. J. Fluid Mech. 374, 285-303 (1998) Zbl 0936.76075 MR 99i:76136

[34] Morrey, C. B.: Multiple Integrals in the Calculus of Variations. Springer, New York (1966) Zbl 0142.38701 MR 34 \#2380

[35] Newell, A. C., Moloney, J. V.: Nonlinear Optics. Addison-Wesley (1992)

[36] Nozières, P., Pines, D.: The Theory of Quantum Liquids, Vol. II: Superfluid Bose Liquids. Perseus Books, Reading, MA (1999)

[37] Rivière, T.: Line vortices in the $U(1)$ Higgs model. ESAIM Control Optim. Calc. Var. 1, 77-167 (1996) Zbl 0874.53019 MR 97g:58043

[38] Roberts, P. H., Berloff, N. G.: The nonlinear Schrödinger equation as a model for superfluidity. Preprint

[39] Simon, L.: Lectures on Geometric Measure Theory. Proc. Centre for Math. Anal., Austr. Nat. Univ. (1983) Zbl 0546.49019 MR 87a:49001

[40] Struwe, M.: On the asymptotic behavior of the Ginzburg-Landau model in 2 dimensions. J. Diff. Equ. 7, 1613-1624 (1994), Erratum 8, 224 (1995) Zbl 0809.35031 MR 95g:35057a

[41] Sulem, C., Sulem, P.-L.: The Nonlinear Schrödinger Equation: Self-Focusing and Wave Collapse. Appl. Math. Sci. 139, Springer (1999) Zbl 0928.35157 $\mid$ MR 2000f:35139 